\title{
FROM THE BGK MODEL TO THE NAVIER-STOKES EQUATIONS
}

\author{
BY LAURE SAINT-RAYMOND
}

ABSTRACT. - We give here a complete derivation of the Navier-Stokes-Fourier equations from a model collisional kinetic equation, the BGK model. Though physically unrealistic, this model shares some common features with more classical models such as the Boltzmann equation.

Then the program developed by Bardos, Golse and Levermore [Fluid dynamic limits of kinetic equations II. Convergence proofs for the Boltzmann equation, Comm. Pure Appl. Math. 46 (5) (1993) 667-753] to study hydrodynamic limits of the steady Boltzmann equation, and extended by Lions and Masmoudi [From Boltzmann equations to Navier-Stokes equations I, Archive Rat. Mech. Anal. 158 (2001) 173-193] in the time-dependent case, can be adapted here, and gives the expected convergence result provided that the particle density $f$ satisfies some integrability assumption.

The originality of the present work is to remove this assumption by establishing refined a priori estimates. The crucial idea is to decompose $f$ as $\left(f-M_{f}\right)+M_{f}$ where $M_{f}$ is the local Maxwellian associated with $f$. The first term is then estimated by means of the entropy dissipation, while the other is smooth in $v$. A mixing property of the operator $\left(\varepsilon \partial_{t}+v \cdot \nabla_{x}\right)$ allows to transfer some of this extra-integrability on the variable $x$.

(C) 2003 Éditions scientifiques et médicales Elsevier SAS

RÉSUMÉ. - On donne ici une dérivation complète des équations de Navier-Stokes-Fourier à partir d'une équation cinétique collisionnelle modèle, l'équation de BGK. Bien que non conforme à la physique, ce modèle présente des propriétés très semblables à celles de l'équation de Boltzmann.

Le programme développé par Bardos, Golse et Levermore [Fluid dynamic limits of kinetic equations II. Convergence proofs for the Boltzmann equation, Comm. Pure Appl. Math. 46 (5) (1993) 667-753] pour étudier les limites hydrodynamiques de l'équation de Boltzmann stationnaire, complété par Lions et Masmoudi [From Boltzmann equations to Navier-Stokes equations I, Archive Rat. Mech. Anal. 158 (2001) 173-193] dans le cas dépendant du temps, peut donc être adapté ici : il donne le résultat de convergence attendu pourvu que la densité de particules $f$ satisfasse une hypothèse d'intégrabilité.

Plusieurs nouvelles idées permettent ici d'établir des estimations a priori précisées et de supprimer cette hypothèse. La plus importante consiste à décomposer $f=\left(f-M_{f}\right)+M_{f}$ où $M_{f}$ est la Maxwellienne locale associée à $f$. Le premier terme est alors estimé au moyen de la dissipation d'entropie, et l'autre est régulier en $v$. Une propriété de mélange de l'opérateur $\left(\varepsilon \partial_{t}+v \cdot \nabla_{x}\right)$ permet de transférer une partie de cette régularité sur la variable $x$.

(c) 2003 Éditions scientifiques et médicales Elsevier SAS

\section{Introduction}

\subsection{From kinetic to macroscopic models}

This paper is devoted to the connection between kinetic theory and macroscopic fluid dynamics. In kinetic theory, a monoatomic gas is represented as a cloud of like point particles 
and is fully described by its number density $f$. The phase space of kinetic theory is the set of $(x, v) \in \mathbb{R}^{d} \times \mathbb{R}^{d}$ where $x$ is the position variable while $v$ is the velocity variable. The meaning of $f$ is as follows: any infinitesimal volume $d x d v$ centered at $(x, v)$ contains at time $t$ about $f(t, x, v) d x d v$ particles. The interaction of particles through collisions is modelled by an operator $C(f)$; this operator acts only on the variable $v$ and is generally nonlinear. If there is neither external force, nor other interaction of particles, the evolution of the density is given by an equation of Boltzmann type

$$
\partial_{t} f+v \cdot \nabla_{x} f=C(f) .
$$

The connection between kinetic and macroscopic fluid dynamics results from two types of properties of the collision operator

(i) the operator $C$ satisfies the usual conservation laws, as well as an entropy relation that implies that the equilibria are Maxwellian distributions;

(ii) the Fréchet-derivative of $C$ at any equilibrium point satisfies a Fredholm alternative with a kernel related to the conservation properties (i).

The macroscopic limits are obtained when the particles undergo many collisions over the scales of interest. Indeed, local equilibrium is reached everywhere, and the fluid is fully described by its moments.

Formal procedures to derive hydrodynamic limits are well-known. They consist in introducing a small parameter $\varepsilon$, called the Knudsen number, that represents the ratio of the mean free path of particles between collisions to some characteristic length of the flow.

$$
\partial_{t} f_{\varepsilon}+v \cdot \nabla_{x} f_{\varepsilon}=\frac{1}{\varepsilon} C\left(f_{\varepsilon}\right) .
$$

Properties (i) are sufficient to derive the compressible Euler equation, assuming a formally consistent convergence for the fluid dynamical moments and entropy of the solutions $f_{\varepsilon}$. The compressible Euler equations also arise as the leading order dynamics from a systematic expansion of $f_{\varepsilon}$ in $\varepsilon$ (the Chapman-Enskog or Hilbert expansion described in [8,9]). Properties (ii) are used to obtain the Navier-Stokes equations; they depend on a more detailed knowledge of the collision operator. The Navier-Stokes equations arise as corrections of those of Euler at the next order in the Chapman-Enskog expansion [9]. Strong hypotheses are needed on the regularity of solutions of the compressible Navier-Stokes equations in order to make sense of these expansions [3].

In order to obtain a fluid dynamical limit with a positive viscosity, the Reynolds number $R e$ which is the reciprocal viscosity of the fluid has to remain finite. From the Von Karman identity $[17,3]$

$$
K n=\varepsilon=\frac{M a}{R e}
$$

where the Mach number $M a$ is defined as the ratio of the bulk velocity to the sound speed, it is clear that such a regime will be necessarily incompressible $(M a \sim \varepsilon)$. In order to realize distributions with a small Mach number, it is natural to consider them as perturbations about a given absolute Maxwellian $M$. A formal procedure leading to the derivation of the incompressible Navier-Stokes equations from

$$
\varepsilon \partial_{t} f_{\varepsilon}+v \cdot \nabla_{x} f_{\varepsilon}=\frac{1}{\varepsilon} C\left(f_{\varepsilon}\right), \quad f_{\varepsilon}=M\left(1+\varepsilon g_{\varepsilon}\right)
$$

is given in [3].

A longstanding mathematical problem is the rigorous justification of such a formal derivation. If the dimension $d$ of the physical space is 3 , the collisional model the most commonly accepted 
is the Boltzmann one. The collision operator takes into account only binary collisions, which are assumed to be elastic; it is given by the formula

$$
C(f)=\int_{\mathbb{R}^{d}} d v_{*} \int_{S^{d-1}} d \omega\left|v-v_{*}\right| \Sigma\left(v-v_{*}, \omega\right)\left(f\left(v^{\prime}\right) f\left(v_{*}^{\prime}\right)-f(v) f\left(v_{*}\right)\right)
$$

where $v^{\prime}$ and $v_{*}^{\prime}$ are given in terms of $v, v_{*}$ and $\omega$ by the classical relations of conservation of mass, momentum and energy [8]. The non-negative function $\Sigma$, the so-called cross-section, is assumed to satisfy some symmetry relations as well as some integrability estimates. In this framework, DiPerna and Lions have established the global existence of solutions - in a very weak sense - of the Boltzmann equation, the so-called renormalized solutions [12]. Such solutions are not known to satisfy the local conservations of momentum and energy. Thus, till now, formal procedures have been completely justified in the large only in some particular regimes: Bardos, Golse and Levermore have proved the convergence towards the acoustic limit [4], and towards the Stokes limit [5]. Other convergence results hold only locally in time: they are based on the Chapman-Enskog expansion method, and are valid as long as the solution of the limiting hydrodynamic model is smooth. Caflish [7] and Nishida [21] have justified in this way the derivation of the compressible Euler equations while De Masi, Esposito and Lebowitz [11] have obtained the incompressible Navier-Stokes equations. Convergences in the large cannot be obtained with such a method, because some hydrodynamic equations, like the compressible Euler system, are known to generate singularities in finite time.

In the range of parameters we will consider in this paper, that is the range of parameters for which the incompressible Navier-Stokes equations are expected to provide a good approximation to the solution of the kinetic equation, partial results have been obtained by Bardos, Golse and Levermore in [4] in the time-discretized case, and extended by Lions and Masmoudi in [20] to the more general time-continuous case. Nevertheless, in both papers, the program remained incomplete. A first difficulty was linked to the concept of renormalized solution: the local conservation of momentum was not established, and had to be assumed

$$
\varepsilon \partial_{t} \int M g_{\varepsilon} v d v+\nabla_{x} \cdot \int M g_{\varepsilon} v \otimes v d v=0
$$

The second assumption which was made in order to derive the evolution equation on the mean velocity is more technical: it was assumed that the family

$$
\left(M \frac{g_{\varepsilon}^{2}}{1+\frac{\varepsilon}{3} g_{\varepsilon}}\left(1+|v|^{2}\right)\right)_{\varepsilon>0}
$$

where $g_{\varepsilon}$ denotes the fluctuation of density $f_{\varepsilon}=M\left(1+\varepsilon g_{\varepsilon}\right)$, is weakly compact in $L_{\text {loc }}^{1}\left(\mathbb{R}^{+}, L^{1}\left(\mathbb{R}^{2 d}\right)\right)$. This assumption allowed on the one hand to control large velocities, and on the other hand brought some compactness with respect to the space variable $x$. In order to fulfill the program, we have to get rid of these hypotheses, and to obtain a complete description of the asymptotic behaviour of the fluctuation $g_{\varepsilon}$ (not only of its mean velocity $\int M g_{\varepsilon} v d v$ ).

We present here such a complete derivation, not for the original Boltzmann equation, but for a simplified model, that is the BGK Boltzmann equation. This process has to be compared with the works of Quastel and Yau on the hydrodynamic limits of Hamiltonian systems. In [24], they study the incompressible limit for a class of stochastic particle systems on the cubic lattice $\mathbb{Z}^{d}$, which are simplified and regularized versions for the Hamiltonian systems: they prove that the limiting distributions of the evolving momentum densities are supported on global weak 
solutions of the Navier-Stokes equations. In the same way, we obtain here Leray's weak solutions directly as the incompressible scaling limit of the BGK kinetic model, which is a relaxation model associated with the Boltzmann equation. Note that several of the ideas introduced here to study this simplified model have been recently re-used in a joint work with Golse [15] to get a complete derivation of the incompressible Navier-Stokes limit of the Boltzmann equation.

\subsection{The BGK Boltzmann equation}

The BGK equation is a model kinetic collisional equation, which can be considered in any dimension $d \geqslant 1$ and which takes into account only the global effect of interactions between fluid particles: such an effect is expected to be a relaxation towards local thermodynamic equilibrium. Then the evolution of the microscopic density $f$ is governed by the nonlinear equation of Boltzmann type

$$
\left\{\begin{array}{l}
\partial_{t} f+v \cdot \nabla_{x} f=\frac{1}{\nu}\left(M_{f}-f\right) \\
M_{f}(t, x, v)=\frac{R(t, x)}{(2 \pi T(t, x))^{d / 2}} \exp \left(-\frac{|v-U(t, x)|^{2}}{2 T(t, x)}\right) \\
R(t, x)=\int f(t, x, v) d v, \quad R U(t, x)=\int f(t, x, v) v d v \\
\quad\left(R U^{2}+d R T\right)(t, x)=\int f(t, x, v)|v|^{2} d v \\
f(0, x, v)=f_{0}(x, v)
\end{array}\right.
$$

where $\nu$ is the relaxation parameter. Note that $U(t, x)$ and $T(t, x)$ are not defined if $R(t, x)=0$, but it does not matter since in this case $M_{f}(t, x, v)=f(t, x, v)=0$ for all $v \in \mathbb{R}^{d}$. Although this model does not contain all the physically relevant features of the classical Boltzmann equation with binary collisions, it has raised the interest of physicists [2,23,29] because it contains most of the basic properties of hydrodynamics. More precisely, we have at least formally the local conservation of mass, momentum and energy

$$
\begin{gathered}
\partial_{t} R+\nabla_{x} \cdot\left(\int f v d v\right)=0, \\
\partial_{t}(R U)+\nabla_{x} \cdot\left(\int f v \otimes v d v\right)=0, \\
\partial_{t}\left(R U^{2}+d R T\right)+\nabla_{x} \cdot\left(\int f v|v|^{2} d v\right)=0,
\end{gathered}
$$

and the decay of entropy

$$
\partial_{t}\left(\int f \log f d v\right)+\nabla_{x} \cdot\left(\int f \log f v d v\right) \leqslant 0
$$

from which we deduce that the equilibria are Maxwellian distributions.

Nevertheless, the BGK Boltzmann model is very simple compared to the Boltzmann equation with binary collisions. First of all, it is not really a collisional model. In particular, in the 
case where $\nu$ is constant, the BGK operator is homogeneous of degree 1 with respect to the macroscopic density while the Boltzmann operator is quadratic. Note that it should matter little in our study, because we expect that

$$
\begin{array}{ll}
R_{\varepsilon}=R+\varepsilon \rho_{\varepsilon}, & \text { where } R \text { is the mean macroscopic density } \\
& \text { and } \rho_{\varepsilon} \text { is uniformly bounded in some convenient space. }
\end{array}
$$

Secondly, we will see that, in the case where $\nu$ is constant, the BGK equation admits global weak solutions without restriction on the size of the initial data, so that the ideas of renormalization introduced for kinetic equations by DiPerna and Lions [12] are useless.

The third simplification will appear often in the paper: as the BGK operator is a relaxation operator, we have a good control on the distance between the density and the corresponding local equilibrium. The tool which allows to estimate this distance, the so-called entropy dissipation, has an equivalent in the case of the classical Boltzmann equation. Various works $[1,28]$ show that the entropy dissipation gives even in the Boltzmann case estimates on the distance between the microscopic density and the corresponding Maxwellian: nevertheless these estimates are not sufficient to justify hydrodynamic limits in the Boltzmann case by the method presented here.

Consider now the scaling for which the incompressible Navier-Stokes equations are expected to provide a good approximation to the solution of the BGK equation. Recall that the Knudsen number $\varepsilon$ has to be small in order to have a macroscopic description of the fluid, and that the Reynolds number $R e$ which measures the viscosity of the fluid has to remain of order 1 . The Von Karman relation implies then that the Mach number $M a$ is of order $\varepsilon$, which is ensured for distributions defined as perturbations about a given absolute Maxwellian $M$. By the proper choice of Galilean frame and dimensional units, this absolute Maxwellian can be taken to have velocity equal to 0 , and density and temperature equal to 1 ; it will be denoted by $M$ :

$$
M(v)=\frac{1}{(2 \pi)^{d / 2}} \exp \left(-\frac{|v|^{2}}{2}\right) .
$$

Thus, in dimensionless variables, the system can be rewritten

$$
\left\{\begin{array}{l}
\varepsilon \partial_{t} f_{\varepsilon}+v \cdot \nabla_{x} f_{\varepsilon}=\frac{1}{\varepsilon \nu}\left(M_{f_{\varepsilon}}-f_{\varepsilon}\right), \\
M_{f_{\varepsilon}}(t, x, v)=\frac{R_{\varepsilon}(t, x)}{\left(2 \pi T_{\varepsilon}(t, x)\right)^{d / 2}} \exp \left(-\frac{\left|v-U_{\varepsilon}(t, x)\right|^{2}}{2 T_{\varepsilon}(t, x)}\right), \\
R_{\varepsilon}(t, x)=\int f_{\varepsilon}(t, x, v) d v, \quad R_{\varepsilon} U_{\varepsilon}(t, x)=\int f_{\varepsilon}(t, x, v) v d v \\
\left(R_{\varepsilon} U_{\varepsilon}^{2}+d R_{\varepsilon} T_{\varepsilon}\right)(t, x)=\int f_{\varepsilon}(t, x, v)|v|^{2} d v, \\
f_{\varepsilon}(0, x, v)=M\left(1+\varepsilon g_{\varepsilon}^{0}(x, v)\right) .
\end{array}\right.
$$

As was noticed in the previous paragraph, the derivation of the Navier-Stokes equations, even the formal one, requires some properties of the operator $C$, namely properties (i) and (ii) above. Furthermore, the form of the limiting Navier-Stokes equations is not independent of the choice of the collision operator within the class of operators satisfying the conservation and the entropy properties. We will see in particular that the Prandtl number (the ratio between the viscosity and the heat conductivity) obtained from the BGK model is always equal to 1 , which is not physically 
relevant. In the case of the Boltzmann equation, this number depends on the cross-section, and therefore on the properties of the fluid, as it is expected. The relaxation BGK model does not behave exactly as the collision model in this regime. Nevertheless, the formal derivation is done in the same way. Introduce the Hilbert space $L^{2}\left(\mathbb{R}^{d}, M d v\right)$ defined by the scalar product

$$
(f, g) \mapsto \int f(v) g(v) M(v) d v .
$$

Because $M d v$ is a positive unit measure on $\mathbb{R}^{d}$, we denote by $\langle\xi\rangle$ the average over this measure of any integrable function $\xi=\xi(v)$

$$
\langle\xi\rangle=\int \xi(v) M(v) d v
$$

Denote by $L$ the first Fréchet derivative of the operator $G \mapsto-M^{-1} C(M G)$ at $G=1$ :

$$
-L(g)=\frac{1}{M} D C(M) \cdot(M g)
$$

In order to obtain an explicit formula for $L$, we perform the following formal computation. By Taylor's formula,

$$
\frac{1}{M} C(M(1+\varepsilon g))=-\varepsilon L(g)+\mathrm{O}\left(\varepsilon^{2}\right) .
$$

Denote respectively by $R_{\varepsilon}, U_{\varepsilon}$ and $T_{\varepsilon}$ the density, the mean velocity and the temperature corresponding to the microscopic density $M(1+\varepsilon g)$. Simple computations show that

$$
R_{\varepsilon}=1+\varepsilon\langle g\rangle, \quad R_{\varepsilon} U_{\varepsilon}=\varepsilon\langle g v\rangle, \quad \text { and } \quad R_{\varepsilon} U_{\varepsilon}^{2}+d R_{\varepsilon} T_{\varepsilon}=d+\varepsilon\left\langle g|v|^{2}\right\rangle
$$

from which we deduce that

$$
U_{\varepsilon}=\varepsilon\langle g v\rangle+\mathrm{O}\left(\varepsilon^{2}\right), \quad \text { and } \quad T_{\varepsilon}=1+\varepsilon\left\langle g\left(|v|^{2} / d-1\right)\right\rangle d v+\mathrm{O}\left(\varepsilon^{2}\right) .
$$

Then,

$$
\begin{aligned}
C(M(1+\varepsilon g)) & =\frac{R_{\varepsilon}}{\left(2 \pi T_{\varepsilon}\right)^{d / 2}} \exp \left(-\frac{\left|v-U_{\varepsilon}\right|^{2}}{2 T_{\varepsilon}}\right)-M(1+\varepsilon g) \\
& =\varepsilon M\left(\langle g\rangle+\langle g v\rangle \cdot v+\left\langle g\left(|v|^{2} / d-1\right)\right\rangle \frac{|v|^{2}-d}{2}-g\right)+\mathrm{O}\left(\varepsilon^{2}\right)
\end{aligned}
$$

which implies that

$$
L(g)=g-\Pi(g),
$$

where $\Pi$ is the projection of $L^{2}\left(\mathbb{R}^{d}, M d v\right)$ on its $(d+2)$-dimensional subset spanned by $\left\{1, v_{1}, \ldots, v_{d},|v|^{2}\right\}$ defined by

$$
\Pi(g)=\langle g\rangle+\langle g v\rangle \cdot v+\left\langle g\left(|v|^{2} / d-1\right)\right\rangle \frac{|v|^{2}-d}{2} .
$$




\subsection{Formal derivation}

Consider a family of solutions of system (1.2). Assume that the fluctuation $g_{\varepsilon}=\varepsilon^{-1}\left(\frac{f_{\varepsilon}}{M}-1\right)$ converges in a weak sense to a function $g$. Then, multiplying the kinetic equation by $\varepsilon$ and letting $\varepsilon$ go to zero yields the relation

$$
L(g)=0 .
$$

This implies that $g$ belongs to the kernel of $L$ and thus can be written according to the formula

$$
g(t, x, v)=\rho(t, x)+u(t, x) \cdot v+\theta(t, x) \frac{|v|^{2}-d}{2} .
$$

The derivation of the Boussinesq and the incompressibility relations start from the equations for conservation of mass and momentum

$$
\begin{gathered}
\varepsilon \partial_{t}\left\langle g_{\varepsilon}\right\rangle+\nabla_{x} \cdot\left\langle g_{\varepsilon} v\right\rangle=0, \\
\varepsilon \partial_{t}\left\langle g_{\varepsilon} v\right\rangle+\nabla_{x} \cdot\left\langle g_{\varepsilon} v \otimes v\right\rangle=0 .
\end{gathered}
$$

Letting $\varepsilon$ go to zero above gives formally

$$
\nabla_{x} \cdot\langle g v\rangle=0, \quad \nabla_{x} \cdot\langle g v \otimes v\rangle=0 .
$$

When $g$ is replaced by the right side of (1.3) these become

$$
\nabla_{x} . u=0, \quad \nabla_{x}(\rho+\theta)=0 .
$$

The limiting momentum equation is obtained from

$$
\partial_{t}\left\langle g_{\varepsilon} v\right\rangle+\frac{1}{\varepsilon} \nabla_{x} \cdot\left\langle g_{\varepsilon} v \otimes v\right\rangle=0
$$

by first separating the flux tensor into its traceless and diagonal parts

$$
\partial_{t}\left\langle g_{\varepsilon} v\right\rangle+\frac{1}{\varepsilon} \nabla_{x} \cdot\left\langle g_{\varepsilon} A(v)\right\rangle=-\nabla_{x} \pi_{\varepsilon}
$$

where $A(v)=v \otimes v-\frac{|v|^{2}}{d} \operatorname{Id}$ and $\pi_{\varepsilon}=\frac{1}{\varepsilon}\left\langle g_{\varepsilon} \frac{|v|^{2}}{d}\right\rangle$. In the same spirit, the limiting temperature equation is obtained by combining the density and energy equations as

$$
\partial_{t}\left\langle g_{\varepsilon}\left(|v|^{2}-(d+2)\right)\right\rangle+\frac{1}{\varepsilon} \nabla_{x} \cdot\left\langle g_{\varepsilon} B(v)\right\rangle=0
$$

where $B(v)=v\left(|v|^{2}-(d+2)\right)$. Assuming the moment convergence as previously, and using the limiting form of $g$ given by (1.3) provide the evaluation of the distribution limits

$$
\left\langle g_{\varepsilon} v\right\rangle \rightarrow u, \quad\left\langle g_{\varepsilon}\left(|v|^{2}-(d+2)\right)\right\rangle \rightarrow(d+2) \theta .
$$

It remains then to estimate the limits of the fluxes $\varepsilon^{-1}\left\langle g_{\varepsilon} A(v)\right\rangle$ and $\varepsilon^{-1}\left\langle g_{\varepsilon} B(v)\right\rangle$. Using the identity $f_{\varepsilon}-M=\left(M_{f_{\varepsilon}}-M\right)+\left(f_{\varepsilon}-M_{f_{\varepsilon}}\right)$, we decompose each of them in two terms, called in the sequel convection and diffusion terms: 


$$
\begin{aligned}
\varepsilon^{-1}\left\langle g_{\varepsilon} A(v)\right\rangle & =\frac{1}{\varepsilon^{2}} \int\left(M_{f_{\varepsilon}}-M\right) A(v) d v+\frac{1}{\varepsilon^{2}} \int\left(f_{\varepsilon}-M_{f_{\varepsilon}}\right) A(v) d v \\
& =\frac{1}{\varepsilon^{2}} R_{\varepsilon} A\left(U_{\varepsilon}\right)-\nu\left\langle v \cdot \nabla_{x} g_{\varepsilon} A(v)\right\rangle+\mathrm{O}(\varepsilon), \\
\varepsilon^{-1}\left\langle g_{\varepsilon} B(v)\right\rangle & =\frac{d+2}{\varepsilon^{2}} R_{\varepsilon}\left(T_{\varepsilon}-1\right) U_{\varepsilon}+\frac{1}{\varepsilon^{2}} R_{\varepsilon} U_{\varepsilon}^{3}-\nu\left\langle v \cdot \nabla_{x} g_{\varepsilon} B(v)\right\rangle+\mathrm{O}(\varepsilon),
\end{aligned}
$$

because $\langle A(v)\rangle=\langle B(v)\rangle=0$. We deduce formally

$$
\begin{aligned}
& \varepsilon^{-1}\left\langle g_{\varepsilon} A(v)\right\rangle \rightarrow A(u)-\nu \nabla_{x} \cdot\langle v g A(v)\rangle, \\
& \varepsilon^{-1}\left\langle g_{\varepsilon} B(v)\right\rangle \rightarrow(d+2) \theta u-\nu \nabla_{x} \cdot\langle v g B(v)\rangle .
\end{aligned}
$$

Using the limiting form of $g$ to compute $\partial_{x_{i}} \partial_{x_{j}}\left\langle v_{i} g A_{j}(v)\right\rangle$ and $\partial_{x_{i}} \partial_{x_{j}}\left\langle v_{i} g B_{j}(v)\right\rangle$, and combining (1.5)-(1.8) provide

$$
\begin{gathered}
\partial_{t} u+\nabla_{x} \cdot(u \otimes u)+\nabla_{x} \Pi=\nu \Delta u, \\
\partial_{t} \theta+\nabla_{x} \cdot(\theta u)=\nu \Delta \theta .
\end{gathered}
$$

The subject matter of this paper is to make the formal derivation above completely rigorous.

\subsection{Statement of main results}

The first step is to give some mathematical framework in which this derivation makes sense. In particular, we have to check that system (1.2) admits a global solution, and that such a solution remains a fluctuation of order $\varepsilon$ around the global Maxwellian $M$. Slight adaptations of the proof of Perthame given in [22] allow to get a good theory of the BGK equation for such perturbations.

For any pair of measurable functions defined and a.e. nonnegative on $\mathbb{R}^{d} \times \mathbb{R}^{d}$, define the relative entropy

$$
H(f / g)=\iint\left(f \log \left(\frac{f}{g}\right)-f+g\right) d v d x \geqslant 0 .
$$

THEOREM 1.1 [26]. - Let $\varepsilon>0$ and $f_{\varepsilon}^{0} \in L_{\text {loc }}^{1}\left(\mathbb{R}^{d} \times \mathbb{R}^{d}\right)$ be a non-negative function such that

$$
H\left(f_{\varepsilon}^{0} / M\right)<+\infty
$$

Then, there exists a global, nonnegative weak solution $f_{\varepsilon}$ to (1.2) which satisfies $f_{\varepsilon}-M \in$ $C\left(\mathbb{R}^{+}, L^{2}\left(\mathbb{R}^{d} \times \mathbb{R}^{d}\right)+L^{1}\left(\mathbb{R}^{d} \times \mathbb{R}^{d}\right)\right)$ and

$$
\forall t>0, \quad H\left(f_{\varepsilon}(t) / M\right)+\frac{1}{\varepsilon^{2} \nu} \int_{0}^{t} \iint D\left(f_{\varepsilon}\right)(s) d v d x d s \leqslant H\left(f_{\varepsilon}^{0} / M\right)
$$

where the dissipation term $D\left(f_{\varepsilon}\right)$ is defined by

$$
D\left(f_{\varepsilon}\right)=\left(M_{f_{\varepsilon}}-f_{\varepsilon}\right) \log \left(\frac{M_{f_{\varepsilon}}}{f_{\varepsilon}}\right) \geqslant 0 .
$$

Furthermore the following local conservation laws hold 


$$
\begin{gathered}
\partial_{t} \int f_{\varepsilon} d v+\frac{1}{\varepsilon} \nabla_{x} \cdot \int v f_{\varepsilon} d v=0, \\
\partial_{t} \int v f_{\varepsilon} d v+\frac{1}{\varepsilon} \nabla_{x} \cdot \int v \otimes v f_{\varepsilon} d v=0, \\
\partial_{t} \int|v|^{2} f_{\varepsilon} d v+\frac{1}{\varepsilon} \nabla_{x} \cdot \int|v|^{2} v f_{\varepsilon} d v=0 .
\end{gathered}
$$

In particular, if the sequence of initial fluctuations $\left(g_{\varepsilon}^{0}\right)$, defined by $g_{\varepsilon}^{0}=\frac{1}{\varepsilon}\left(\frac{f_{\varepsilon}^{0}}{M}-1\right)$, is bounded in $L^{2}\left(\mathbb{R}^{d} \times \mathbb{R}^{d}, d x M d v\right)$, it is proved in [4] that the entropy bound

$$
\frac{1}{\varepsilon^{2}} H\left(f_{\varepsilon} / M\right) \leqslant \frac{1}{\varepsilon^{2}} H\left(M\left(1+\varepsilon g_{\varepsilon}^{0}\right) / M\right) \leqslant C_{0}
$$

provides weak compactness on $\left(M g_{\varepsilon}\right)$ in $L_{\text {loc }}^{1}\left(\mathbb{R}_{t}^{+} \times \mathbb{R}_{x}^{d}, L^{1}\left(\mathbb{R}_{v}^{d}\right)\right)$.

In this framework, it is meaningful to ask whether the moments of the limiting fluctuation satisfy the incompressible Navier-Stokes equations. Before stating the main result, let introduce the abbreviations $w-L^{p}$ for the weak topology $\sigma\left(L^{p}, L^{p^{\prime}}\right)$ if $p<+\infty$, and $w-L^{\infty}$ for the weak-* topology $\sigma\left(L^{\infty}, L^{1}\right)$.

THEOREM 1.2. - Let $\left(g_{\varepsilon}^{0}\right)$ be a family of measurable functions on $\mathbb{R}^{d} \times \mathbb{R}^{d}$ satisfying

$$
1+\varepsilon g_{\varepsilon}^{0} \geqslant 0 \text { a.e., } \quad H\left(M\left(1+\varepsilon g_{\varepsilon}^{0}\right) / M\right) \leqslant C_{0} \varepsilon^{2},
$$

as well as

$$
\begin{aligned}
& \left\langle g_{\varepsilon}^{0} v\right\rangle \rightarrow u_{0} \text { with } \nabla_{x} . u_{0}=0 \quad \text { in } w-L^{2}\left(\mathbb{R}^{d}\right) \text { as } \varepsilon \rightarrow 0, \\
& \left\langle g_{\varepsilon}^{0}\left(\frac{|v|^{2}}{d+2}-1\right)\right\rangle \rightarrow \theta_{0} \quad \text { in } w-L^{2}\left(\mathbb{R}^{d}\right) \text { as } \varepsilon \rightarrow 0 .
\end{aligned}
$$

Denote by $f_{\varepsilon}=M\left(1+\varepsilon g_{\varepsilon}\right)$ a solution of (1.2). Then there exist

$$
\rho, u, \theta \in L^{\infty}\left(\mathbb{R}^{+}, L^{2}\left(\mathbb{R}^{d}\right)\right) \cap L^{2}\left(\mathbb{R}^{+}, H^{1}\left(\mathbb{R}^{d}\right)\right)
$$

such that, up to extraction of a sequence $\varepsilon_{n}$ going to zero,

$$
\begin{aligned}
& \left\langle g_{\varepsilon}\right\rangle \rightarrow \rho \quad \text { in } w-L_{\mathrm{loc}}^{1}\left(\mathbb{R}^{+} \times \mathbb{R}^{d}\right) \text { as } \varepsilon \rightarrow 0, \\
& \left\langle g_{\varepsilon} v\right\rangle \rightarrow u \quad \text { in } w-L_{\mathrm{loc}}^{1}\left(\mathbb{R}^{+} \times \mathbb{R}^{d}\right) \text { as } \varepsilon \rightarrow 0, \\
& \left\langle g_{\varepsilon}\left(\frac{|v|^{2}}{d}-1\right)\right\rangle \rightarrow \theta \quad \text { in } w-L_{\mathrm{loc}}^{1}\left(\mathbb{R}^{+} \times \mathbb{R}^{d}\right) \text { as } \varepsilon \rightarrow 0,
\end{aligned}
$$

and $\rho$, $u$ and $\theta$ satisfy the Navier-Stokes-Fourier equations

$$
\left\{\begin{array}{l}
\partial_{t} u+\nabla_{x} \cdot(u \otimes u)+\nabla_{x} \pi=\nu \Delta u, \quad \nabla_{x} . u=0, \\
\partial_{t} \theta+\nabla_{x} .(\theta u)=\nu \Delta \theta, \quad \rho+\theta=0, \\
u(0, .)=u_{0}, \quad \theta(0, .)=\theta_{0}
\end{array}\right.
$$

where $\pi: \mathbb{R}^{+} \times \mathbb{R}^{d} \rightarrow \mathbb{R}$, the so-called pressure, is prescribed by the incompressibility constraint.

\section{Outline of the convergence proof}

For the sake of clarity, we will take $\nu=1$ in all the sequel. 


\subsection{Entropy estimates}

As said in the introduction a basic tool to study hydrodynamic limits is the relative entropy. Define the positive convex function

$$
h: z \in]-1,+\infty[\mapsto h(z)=(1+z) \log (1+z)-z .
$$

The classical entropy estimate (1.11), the so-called H-Theorem, coupled with the scaling assumption (1.13), shows that

$$
\forall t>0, \quad \varepsilon^{-2} \int\left\langle h\left(\varepsilon g_{\varepsilon}(t)\right)\right\rangle d x \leqslant C_{0} .
$$

Since $h(z) \sim \frac{1}{2} z^{2}$ near $z=0$, the entropy control is as good as a $L^{\infty}\left(\mathbb{R}^{+}, L^{2}\left(\mathbb{R}^{2 d}, d x M d v\right)\right)$ control but only for the part of $g_{\varepsilon}$ that does not exceed $1 / \varepsilon$ in size. This suggests the decomposition of Hilbert type $f_{\varepsilon}=M\left(1+\varepsilon^{b} g_{\varepsilon}+\varepsilon^{2 \sharp} g_{\varepsilon}\right)$ with

$$
{ }^{b} g_{\varepsilon}=g_{\varepsilon} \gamma\left(1+\varepsilon g_{\varepsilon}\right), \quad{ }^{\sharp} g_{\varepsilon}=\frac{1}{\varepsilon} g_{\varepsilon}\left(1-\gamma\left(1+\varepsilon g_{\varepsilon}\right)\right)
$$

and

$$
\gamma \in C^{\infty}\left(\mathbb{R}^{+}\right) \text {is supported in }\left[\frac{1}{2}, \frac{3}{2}\right], \text { and satisfies } \gamma\left(\left[\frac{3}{4}, \frac{5}{4}\right]\right)=\{1\} .
$$

This decomposition is not exactly the same as the one given in [4], but it shares most of its key properties. Moreover, with the present definition, the main part of the fluctuation ${ }^{b} g_{\varepsilon}$ is expected to contain all the informations leading to the Navier-Stokes asymptotic. A priori entropy-based estimates on ${ }^{b} g_{\varepsilon}$ and $\sharp g_{\varepsilon}$ will be detailed in Section 3 .

Nevertheless we are not able to derive rigorously the Navier-Stokes asymptotic using only these estimates. In order to take limits in the fluxes $\varepsilon^{-1}\left\langle g_{\varepsilon} A(v)\right\rangle$ and $\varepsilon^{-1}\left\langle g_{\varepsilon} B(v)\right\rangle$ and to establish a statement like (1.8), a natural idea is to decompose the fluctuation $g_{\varepsilon}$ by (2.2). We then deal with the main part as in the above formal derivation: the identity

$$
{ }^{b} g_{\varepsilon}=\Pi^{b} g_{\varepsilon}+\left({ }^{b} g_{\varepsilon}-\Pi^{b} g_{\varepsilon}\right)=\Pi^{b} g_{\varepsilon}+L^{b} g_{\varepsilon}
$$

allows to decompose each flux in a convection term and a diffusion term. The difficulty is therefore to obtain the convergence of the convection term, which should require strong compactness in space and time on the moments of ${ }^{b} g_{\varepsilon}$. The former will be a consequence of averaging lemma [14], and an extension of the results of [20] will show that the presence of high frequency oscillations contributes only to the limiting pressure term, but only provided that

$$
\left(M^{\mathrm{b}} g_{\varepsilon}^{2}\right) \text { is relatively compact in } w-L_{\text {loc }}^{1}\left(\mathbb{R}^{+} \times \mathbb{R}_{x}^{d}, L^{1}\left(\mathbb{R}_{v}^{d}\right)\right) \text {. }
$$

It remains then to obtain the convergence of the remainder, i.e. to establish that ${ }^{\sharp} g_{\varepsilon}$ converges to 0 and does not bring any contribution to the limiting equation, which should hold if

$$
\left(M^{\sharp} g_{\varepsilon}\left(1+|v|^{2}\right)\right) \text { is relatively compact in } w-L_{\text {loc }}^{1}\left(\mathbb{R}^{+} \times \mathbb{R}_{x}^{d}, L^{1}\left(\mathbb{R}_{v}^{d}\right)\right) .
$$

Both statements (E1) and (E2) were consequences in [4] and [20] of the assumption (H2). Here (E1) and a weak form of (E2) will be established using the entropy dissipation bound and some properties of the advection operator $\left(\varepsilon \partial_{t}+v \cdot \nabla_{x}\right)$ which have already allowed to relax assumption (H2) in a previous paper on the BGK model [26]. 


\subsection{Dissipation estimates}

A first remark is that the H-theorem (1.11) provides another essential bound

$$
\forall t>0, \quad \frac{1}{\varepsilon^{4}} \int_{0}^{t} \iint D\left(f_{\varepsilon}\right)(s) d v d x d s=\frac{1}{\varepsilon^{4}} \int_{0}^{t} \iint M_{f_{\varepsilon}} r\left(\frac{f_{\varepsilon}}{M_{f_{\varepsilon}}}-1\right)(s) d s d x d v \leqslant C_{0}
$$

where $r$ denotes the positive convex function

$$
r: z \in]-1,+\infty[\mapsto r(z)=z \log (1+z) .
$$

Since $r(z) \sim \frac{1}{2} z^{2}$ near $z=0$ and because we expect $M_{f_{\varepsilon}}$ to be close to $M$, this indicates that

$$
q_{\varepsilon}=\frac{f_{\varepsilon}-M_{f_{\varepsilon}}}{\varepsilon^{2} M_{f_{\varepsilon}}}
$$

is bounded in a convenient space which is almost as good as $L^{2}\left(\mathbb{R}^{+} \times \mathbb{R}^{2 d}, d t d x M d v\right)$. It seems then natural to introduce the decomposition of Chapman-Enskog type

$$
f_{\varepsilon}=M_{f_{\varepsilon}}\left(1+\varepsilon^{2} q_{\varepsilon}\right)=M_{f_{\varepsilon}}\left(1+\varepsilon^{2 b} q_{\varepsilon}+\varepsilon^{4 \sharp} q_{\varepsilon}\right)
$$

with

$$
{ }^{b} q_{\varepsilon}=q_{\varepsilon} \gamma\left(1+\varepsilon^{2} q_{\varepsilon}\right), \quad{ }^{\sharp} q_{\varepsilon}=\frac{1}{\varepsilon^{2}} q_{\varepsilon}\left(1-\gamma\left(1+\varepsilon^{2} q_{\varepsilon}\right)\right)
$$

and $\gamma$ defined by (2.3). Note that $M_{f_{\varepsilon}}$ is expected to be very regular with respect to the velocity variables, at least if the moments of $f_{\varepsilon}$ (and consequently of $M_{f_{\varepsilon}}$ ) do not deviate too much from their mean values. In Section 4, we will deduce from (2.4) and (2.5) precise a priori bounds on $g_{\varepsilon}$, called dissipation-based estimates.

These estimates are not yet sufficient to establish (E1) and (E2), even in a weak form. They improve the $L^{p}$-regularity in the variable $v$, but it remains to transfer some of this extra $L^{p}$-regularity on the variable $x$. Doing so rests on a dispersion property of the operator $\left(\varepsilon \partial_{t}+v \cdot \nabla_{x}\right)$.

\subsection{Interpolation estimates}

The result which will be used here to improve the $L^{p}$-regularity in the variable $x$ is a variant of the following

PROPOSITION 2.1 [26]. - Let $\left(g_{n}\right)$ be a bounded sequence of $L^{1}\left(\mathbb{R}^{d} \times \mathbb{R}^{d}\right)$ such that $\left(v . \nabla_{x} g_{n}\right)$ is bounded in $L^{1}\left(\mathbb{R}^{d} \times \mathbb{R}^{d}\right)$. Assume that $g_{n}$ is equiintegrable with respect to $v$, i.e. that there exists $\left(k_{n}\right)$ a bounded sequence of $L^{1}\left(\mathbb{R}^{d}\right)$ such that

$$
\forall \delta>0, \exists \alpha>0, \forall x, n, O /|O| \leqslant \alpha, \quad \int_{O}\left|g_{n}(x, v)\right| d v \leqslant \delta k_{n}(x) .
$$

Then the sequence $\left(g_{n}\right)$ is equiintegrable (with respect to all variables $x$ and $v$ ). 
The equiintegrability in $x$ can be proved by optimizing in $\tau$ in the formula

$$
\iint g_{n} \mathbb{1}_{A}(x) d x d v=\iint g_{n} \Phi(\tau, \cdot, \cdot) d x d v-\tau \int_{0}^{1} \iint\left(v \cdot \nabla_{x} g_{n}\right) \Phi(s \tau, . ., .) d s d x d v
$$

where the function $\Phi \equiv \Phi(\tau, x, v)$ is the solution of the Cauchy problem for the free transport equation

$$
\partial_{\tau} \Phi+v \cdot \nabla_{x} \Phi=0 \quad \text { for }(\tau, x, v) \in \mathbb{R} \times \mathbb{R}^{d} \times \mathbb{R}^{d}
$$

with $\Phi(0, x, v)=\mathbb{1}_{A}(x)$.

The second term in the right-side is controlled thanks to the uniform $L^{1}$-bound on $v \cdot \nabla_{x} g_{n}$, while the first one is estimated in terms of $|A|=\int \mathbb{1}_{A}(x) d x$ by using the classical dispersion estimates for the free transport equation $[10,6]$

$$
\|\Phi(\tau, ., .)\|_{L_{x}^{q}\left(L_{v}^{1}\right)} \leqslant \tau^{-d\left(1-\frac{1}{q}\right)}\|\Phi(0, . .)\|_{L_{x}^{1}\left(L_{v}^{q}\right)}
$$

and the equiintegrability in $v$.

Combining the equiintegrability in $v$ coming from (2.5) with a local version of the above dispersion result, we will prove in Section 5 a variant of (E1) and (E2), namely

THEOREM 2.2. - Let $\left(g_{\varepsilon}^{0}\right)$ be a family of measurable functions on $\mathbb{R}^{d} \times \mathbb{R}^{d}$ satisfying (1.13) and (1.14). For each $\varepsilon>0$, consider a solution $f_{\varepsilon}=M\left(1+\varepsilon g_{\varepsilon}\right)$ of (1.2), and define ${ }^{b} g_{\varepsilon}$ and ${ }^{\sharp} g_{\varepsilon}$ by (2.2) where $\gamma$ denotes any bump function satisfying (2.3). Then

(i) Up to extraction of a sequence, $\left(M^{b} g_{\varepsilon}^{2}\right)$ is relatively compact in $w-L_{\mathrm{loc}}^{1}\left(\mathbb{R}^{+} \times \mathbb{R}^{d}\right.$, $\left.L^{1}\left(\mathbb{R}^{d}\right)\right)$,

(ii) $M^{\sharp} g_{\varepsilon} \rightarrow 0$ in $L_{\text {loc }}^{1}\left(\mathbb{R}^{+} \times \mathbb{R}^{d}, L^{1}\left(\mathbb{R}^{d}\right)\right)$.

\subsection{The chain-rule for ${ }^{b} g_{\varepsilon}$}

These a priori estimates will be sufficient to derive the Navier-Stokes asymptotic, but not directly from the local conservations of momentum and energy. Indeed they do not provide weak compactness on $\left(M^{\sharp} g_{\varepsilon}|v|^{p}\right)$ for $p \geqslant 2$; in particular, we are not able to take limits in $\left\langle{ }^{\sharp} g_{\varepsilon} A(v)\right\rangle$ and $\left\langle{ }^{\sharp} g_{\varepsilon} B(v)\right\rangle$, and to justify (1.8). Then, in order to overcome such a difficulty, we will consider the chain-rule for ${ }^{b} g_{\varepsilon}$ and estimate the conservation defects for the associated moments. In Section 6, we will establish the following

THEOREM 2.3. - Let $\left(g_{\varepsilon}^{0}\right)$ be a family of measurable functions on $\mathbb{R}^{d} \times \mathbb{R}^{d}$ satisfying (1.13) and (1.14). For each $\varepsilon>0$, consider a solution $f_{\varepsilon}=M\left(1+\varepsilon g_{\varepsilon}\right)$ of (1.2), and define ${ }^{b} g_{\varepsilon}$ by (2.2) where $\gamma$ denotes any bump function satisfying (2.3). Then

$$
\begin{gathered}
\partial_{t}\left\langle{ }^{b} g_{\varepsilon}\right\rangle+\frac{1}{\varepsilon} \nabla_{x} \cdot\left\langle{ }^{b} g_{\varepsilon} v\right\rangle \rightarrow 0 \quad \text { in } L_{\text {loc }}^{1}\left(\mathbb{R}^{+} \times \mathbb{R}^{d}\right), \\
\partial_{t}\left\langle{ }^{b} g_{\varepsilon} v\right\rangle+\frac{1}{\varepsilon} \nabla_{x} \cdot\left\langle{ }^{b} g_{\varepsilon} v \otimes v\right\rangle \rightarrow 0 \quad \text { in } L_{\text {loc }}^{1}\left(\mathbb{R}^{+} \times \mathbb{R}^{d}\right), \\
\partial_{t}\left\langle{ }^{b} g_{\varepsilon}|v|^{2}\right\rangle+\frac{1}{\varepsilon} \nabla_{x} \cdot\left\langle{ }^{b} g_{\varepsilon} v|v|^{2}\right\rangle \rightarrow 0 \quad \text { in } L_{\text {loc }}^{1}\left(\mathbb{R}^{+} \times \mathbb{R}^{d}\right) .
\end{gathered}
$$

Note that using such a method we do not mind whether the local conservation laws hold for fixed $\varepsilon>0$ or not: we recover the mass, motion and heat equations in the limit by controlling 
the local conservation defects. Thus a similar method can be used to study hydrodynamic limits for the renormalized solutions of the Boltzmann equation, which has been done successfully by Golse and Levermore in the acoustic and Stokes regimes [13], and more recently by Golse and the author in the Navier-Stokes regime [15].

\subsection{Passage to the limit}

Equipped with the previous tools, we will achieve in Section 7 the convergence proof sketched in [4]. We will start by proving that the limiting fluctuation belongs to the kernel of the linearized collision operator, i.e. is an infinitesimal Maxwellian. Then we will establish the incompressibility and Boussinesq relations. The last step will be to take limits in (2.8)-(2.10). Using decomposition (2.5) shows that each one of the flux terms $\frac{1}{\varepsilon} \nabla_{x} \cdot\left\langle{ }^{b} g_{\varepsilon}\left(v \otimes v-|v|^{2} / d\right)\right\rangle$ and $\frac{1}{\varepsilon} \nabla_{x} \cdot\left\langle{ }^{b} g_{\varepsilon} v|v|^{2}\right\rangle$ can be split in a diffusion term, a convection term (which is quadratic in the moments of ${ }^{b} g_{\varepsilon}$ ), and a remainder. Using Theorem 2.2 and the compactness arguments given [4] and [20], we will establish the convergence of the convection term which is the most delicate part of the proof.

\section{Entropy-based estimates}

For any Borel measure $\mu$ on $\mathbb{R}^{n}$, we denote by $L^{p}(d \mu)$ the Lebesgue space $L^{p}\left(\mathbb{R}^{n}, d \mu\right)$; the notation $L_{x}^{p}$ designates $L^{p}\left(\mathbb{R}^{n}, d x\right)$ where $d x$ is the $n$-dimensional Lebesgue measure.

\subsection{Basic estimates on ${ }^{b} g_{\varepsilon}$ and ${ }^{\sharp} g_{\varepsilon}$}

LEMMA 3.1. - Let $\left(g_{\varepsilon}^{0}\right)$ be a family of measurable functions on $\mathbb{R}^{d} \times \mathbb{R}^{d}$ satisfying (1.13) and (1.14). For each $\varepsilon>0$, consider a solution $f_{\varepsilon}=M\left(1+\varepsilon g_{\varepsilon}\right)$ of (1.2), and define ${ }^{b} g_{\varepsilon}$ and ${ }^{\sharp} g_{\varepsilon}$ by (2.2) where $\gamma$ denotes any bump function satisfying (2.3). Then there exists a nonnegative constant $C$ such that, for all $\varepsilon>0$,

(i) $\left\|g_{\varepsilon}\right\|_{L^{\infty}\left(d t, L^{2}(d x M d v)\right)} \leqslant C,\left\|\sharp g_{\varepsilon}\right\|_{L^{\infty}\left(d t, L^{1}(d x M d v)\right)} \leqslant C$,

(ii) $\forall \lambda \geqslant 2 e^{2},\left\|\frac{1}{\varepsilon} g_{\varepsilon} \mathbb{1}_{\varepsilon g_{\varepsilon} \geqslant \lambda}\right\|_{L^{\infty}\left(d t, L^{1}(d x M d v)\right)} \leqslant C / \log (1+\lambda)$,

(iii) $\left|{ }^{b} g_{\varepsilon}\right| \leqslant \frac{\|\gamma\|_{\infty}}{2 \varepsilon},\left|1-\gamma\left(f_{\varepsilon} / M\right)\right|\left(f_{\varepsilon}+M\right) \leqslant\left. 9 \varepsilon^{2} M\right|^{\sharp} g_{\varepsilon} \mid$ for almost every $(t, x, v)$.

Proof. - Both assertions (i) and (ii) are direct consequences of the entropy bound (2.1), while (iii) gives pointwise estimates coming from (2.3).

(i) From the elementary inequalities

$$
\left.\left.z^{2} \leqslant C_{1} h(z) \text { for }|z| \leqslant \frac{1}{2}, \quad 1+|z| \leqslant C_{1} h(z) \text { for } z \in\right]-1,-\frac{1}{4}\right] \cup\left[\frac{1}{4},+\infty[\right.
$$

which hold for some constant $C_{1}>0$, we deduce that

$$
\varepsilon^{2 b} g_{\varepsilon}^{2} \leqslant C_{1} h\left(\varepsilon g_{\varepsilon}\right)\|\gamma\|_{\infty}^{2}, \quad \varepsilon^{2}\left|{ }^{\sharp} g_{\varepsilon}\right| \leqslant C_{1} h\left(\varepsilon g_{\varepsilon}\right)\|1-\gamma\|_{\infty} .
$$

Combining (2.1), (2.3) and (3.2) leads then to the expected bounds.

(ii) Observe that the function $z \mapsto \frac{h(z)}{z}$ is increasing on $\mathbb{R}^{+}$. Then, for $\lambda \geqslant 2 e^{2}$,

$$
\varepsilon g_{\varepsilon} \mathbb{1}_{\varepsilon g_{\varepsilon} \geqslant \lambda} \leqslant h\left(\varepsilon g_{\varepsilon}\right) \frac{\lambda}{h(\lambda)} \leqslant h\left(\varepsilon g_{\varepsilon}\right) \frac{1}{\log (1+\lambda)-1} \leqslant h\left(\varepsilon g_{\varepsilon}\right) \frac{2}{\log (1+\lambda)}
$$

which coupled with (2.1) gives the second control. 
(iii) $\operatorname{As} \operatorname{supp}(\gamma) \subset\left[\frac{1}{2}, \frac{3}{2}\right]$ and $\operatorname{supp}(1-\gamma) \subset\left[0, \frac{3}{4}\right] \cup\left[\frac{5}{4},+\infty[\right.$,

$$
\begin{aligned}
& \left.\varepsilon\right|^{b} g_{\varepsilon}|\leqslant \varepsilon| g_{\varepsilon}|| \gamma\left(1+\varepsilon g_{\varepsilon}\right) \mid \leqslant \frac{1}{2}\|\gamma\|_{\infty}, \\
& \varepsilon^{2}\left|{ }^{\sharp} g_{\varepsilon}\right| \geqslant \varepsilon\left|g_{\varepsilon}\right|\left|1-\gamma\left(1+\varepsilon g_{\varepsilon}\right)\right| \geqslant \frac{\left(1+\varepsilon g_{\varepsilon}\right)+1}{9}\left|1-\gamma\left(1+\varepsilon g_{\varepsilon}\right)\right| .
\end{aligned}
$$

Note that combining (i) and (iii) provides a uniform bound on $\frac{1}{\varepsilon^{2}}\left(f_{\varepsilon}+M\right)\left(1-\gamma\left(1+\varepsilon g_{\varepsilon}\right)\right)$ in $L_{t}^{\infty}\left(L_{x, v}^{1}\right)$.

\subsection{Estimates on the moments $R_{\varepsilon}, U_{\varepsilon}$ and $T_{\varepsilon}$}

LEMMA 3.2. - Let $\left(g_{\varepsilon}^{0}\right)$ be a family of measurable functions on $\mathbb{R}^{d} \times \mathbb{R}^{d}$ satisfying (1.13) and (1.14). For each $\varepsilon>0$, consider a solution $f_{\varepsilon}=M\left(1+\varepsilon g_{\varepsilon}\right)$ of (1.2). Denote by $k$ the convex function $z \in]-1,+\infty\left[\mapsto z-\log (1+z)\right.$. Define $\chi_{\varepsilon}$ as the indicator function of $\left\{(t, x)|| R_{\varepsilon}(t, x)-1\left|\leqslant \frac{1}{2},\right| U_{\varepsilon}(t, x)\left|\leqslant \frac{1}{2},\right| T_{\varepsilon}(t, x)-1 \mid \leqslant \frac{1}{2}\right\}$. Then there exists a nonnegative constant $C$ such that

(i)

$$
\sup _{t \geqslant 0} \int\left(h\left(R_{\varepsilon}-1\right)+\frac{1}{2} R_{\varepsilon} U_{\varepsilon}^{2}+\frac{d}{2} R_{\varepsilon} k\left(T_{\varepsilon}-1\right)\right) d x \leqslant C_{0} \varepsilon^{2}
$$

(ii)

$$
\begin{aligned}
& \left\|\chi \chi_{\varepsilon} \frac{R_{\varepsilon}-1}{\varepsilon}\right\|_{L_{t}^{\infty}\left(L_{x}^{2}\right)}+\left\|\chi \chi_{\varepsilon} \frac{U_{\varepsilon}}{\varepsilon}\right\|_{L_{t}^{\infty}\left(L_{x}^{2}\right)}+\left\|\chi_{\varepsilon} \frac{T_{\varepsilon}-1}{\varepsilon}\right\|_{L_{t}^{\infty}\left(L_{x}^{2}\right)} \leqslant C, \\
& \left\|\frac{1-\chi_{\varepsilon}}{\varepsilon^{2}}\left(1+R_{\varepsilon}+R_{\varepsilon} U_{\varepsilon}^{2}+R_{\varepsilon} T_{\varepsilon}\right)\right\|_{L_{t}^{\infty}\left(L_{x}^{1}\right)} \leqslant C .
\end{aligned}
$$

Proof. - By definition of $M_{f_{\varepsilon}}$,

$$
\begin{aligned}
H\left(M_{f_{\varepsilon}} / M\right) & =\iint_{\mathbb{R}^{d} \times \mathbb{R}^{d}}\left(M_{f_{\varepsilon}} \log \frac{M_{f_{\varepsilon}}}{M}-M_{f_{\varepsilon}}+M\right) d x d v \\
& =\iint_{\mathbb{R}^{d} \times \mathbb{R}^{d}}\left(f_{\varepsilon} \log \frac{M_{f_{\varepsilon}}}{M}-M_{f_{\varepsilon}}+M\right) d x d v \\
& =\iint_{\mathbb{R}^{d} \times \mathbb{R}^{d}}\left(\left(f_{\varepsilon} \log \frac{f_{\varepsilon}}{M}-f_{\varepsilon}+M\right)-\left(f_{\varepsilon} \log \frac{f_{\varepsilon}}{M_{f_{\varepsilon}}}-M_{f_{\varepsilon}}+f_{\varepsilon}\right)\right) d x d v \\
& =H\left(f_{\varepsilon} / M\right)-H\left(f_{\varepsilon} / M_{f_{\varepsilon}}\right) \leqslant H\left(f_{\varepsilon} / M\right)
\end{aligned}
$$

which, coupled with (2.1), provides

$$
\sup _{t \geqslant 0} H\left(M_{f_{\varepsilon}} / M\right) \leqslant C_{0} \varepsilon^{2} .
$$

The inequality (i) follows from (3.5) and the elementary formula

$$
H\left(M_{f_{\varepsilon}} / M\right)=\int\left(h\left(R_{\varepsilon}-1\right)+\frac{1}{2} R_{\varepsilon} U_{\varepsilon}^{2}+\frac{d}{2} R_{\varepsilon} k\left(T_{\varepsilon}-1\right)\right) d x .
$$


As for (ii), it is similar to the first assertion in Lemma 3.1. Similarly to (3.1),

$$
\left.\left.z^{2} \leqslant C_{2} k(z) \quad \text { for }|z| \leqslant \frac{1}{2}, \quad 1+|z| \leqslant C_{2} k(z) \quad \text { for } z \in\right]-1,-\frac{1}{4}\right] \cup\left[\frac{1}{4},+\infty[\right.
$$

which hold for some constant $C_{2}>0$. The first statement comes from (i) and the inequality

$$
\chi_{\varepsilon}\left(\left|R_{\varepsilon}-1\right|^{2}+\left|U_{\varepsilon}\right|^{2}+\left|T_{\varepsilon}-1\right|^{2}\right) \leqslant \chi_{\varepsilon}\left(C_{1} h\left(R_{\varepsilon}-1\right)+2 R_{\varepsilon}\left|U_{\varepsilon}\right|^{2}+2 C_{2} R_{\varepsilon} k\left(T_{\varepsilon}-1\right)\right)
$$

that follows from (3.1) and (3.7). In order to establish the second statement, we first prove that the size of the support of $\left(1-\chi_{\varepsilon}\right)$ is of order $\varepsilon^{2}$. Indeed, by (3.1),

$$
\begin{gathered}
\int \mathbb{1}_{\left|R_{\varepsilon}-1\right| \geqslant 1 / 2} d x \leqslant C_{1} \int h\left(R_{\varepsilon}-1\right) \mathbb{1}_{\left|R_{\varepsilon}-1\right| \geqslant 1 / 2} d x \leqslant C_{1} C_{0} \varepsilon^{2}, \\
\int \mathbb{1}_{\left|R_{\varepsilon}-1\right| \leqslant 1 / 2} \mathbb{1}_{\left|U_{\varepsilon}\right| \geqslant 1 / 2} d x \leqslant 8 \int R_{\varepsilon} U_{\varepsilon}^{2} \mathbb{1}_{\left|R_{\varepsilon}-1\right| \leqslant 1 / 2} \mathbb{1}_{\left|U_{\varepsilon}\right| \geqslant 1 / 2} d x \leqslant 16 C_{0} \varepsilon^{2},
\end{gathered}
$$

while by (3.7),

$$
\begin{aligned}
\int \mathbb{1}_{\left|R_{\varepsilon}-1\right| \leqslant 1 / 2} \mathbb{1}_{\left|T_{\varepsilon}-1\right| \geqslant 1 / 2} d x & \leqslant 2 C_{2} \int R_{\varepsilon} k\left(T_{\varepsilon}-1\right) \mathbb{1}_{\left|R_{\varepsilon}-1\right| \leqslant 1 / 2} \mathbb{1}_{\left|T_{\varepsilon}-1\right| \geqslant 1 / 2} d x \\
& \leqslant \frac{4}{d} C_{2} C_{0} \varepsilon^{2}
\end{aligned}
$$

from which we deduce that

$$
\sup _{t \geqslant 0} \int\left(1-\chi_{\varepsilon}\right) d x \leqslant C_{0}\left(C_{1}+16+\frac{4}{d} C_{2}\right) \varepsilon^{2} .
$$

By (3.1) and (3.7),

$$
1+z \leqslant \frac{3}{2}+C_{1} h(z), \quad 1+z \leqslant \frac{3}{2}+C_{2} k(z) \text { for } z>-1 .
$$

Then

$$
\begin{aligned}
\left(1-\chi_{\varepsilon}\right)\left(1+R_{\varepsilon}+R_{\varepsilon} U_{\varepsilon}^{2}+R_{\varepsilon} T_{\varepsilon}\right) \\
\quad \leqslant\left(1-\chi_{\varepsilon}\right)\left(1+R_{\varepsilon}+R_{\varepsilon} U_{\varepsilon}^{2}+R_{\varepsilon}\left(\frac{3}{2}+C_{2} k\left(T_{\varepsilon}-1\right)\right)\right) \\
\quad \leqslant\left(1-\chi_{\varepsilon}\right)\left(1+\frac{5}{2}\left(\frac{3}{2}+C_{1} h\left(R_{\varepsilon}-1\right)\right)+R_{\varepsilon} U_{\varepsilon}^{2}+C_{2} R_{\varepsilon} k\left(T_{\varepsilon}-1\right)\right) \\
\quad \leqslant \frac{19}{4}\left(1-\chi_{\varepsilon}\right)+\left(\frac{5}{2} C_{1} h\left(R_{\varepsilon}-1\right)+R_{\varepsilon} U_{\varepsilon}^{2}+C_{2} R_{\varepsilon} k\left(T_{\varepsilon}-1\right)\right)
\end{aligned}
$$

which, coupled with (3.9) and (i), provides the expected bound. 


\section{Dissipation-based estimates}

4.1. Basic estimates on $M_{f_{\varepsilon}}-f_{\varepsilon}$

LEMMA 4.1. - Let $\left(g_{\varepsilon}^{0}\right)$ be a family of measurable functions on $\mathbb{R}^{d} \times \mathbb{R}^{d}$ satisfying (1.13) and (1.14). For each $\varepsilon>0$, consider a solution $f_{\varepsilon}=M\left(1+\varepsilon g_{\varepsilon}\right)$ of (1.2). Denote by $\gamma$ any bump function satisfying (2.3). Then,

(i) For all $t^{*}>0$, there exists a nonnegative constant $C$ such that, for all $\varepsilon>0$,

$$
\begin{aligned}
& \left\|\frac{1}{\varepsilon^{2}} \frac{M_{f_{\varepsilon}}-f_{\varepsilon}}{f_{\varepsilon}^{1 / 2}} \gamma\left(\frac{f_{\varepsilon}}{M_{f_{\varepsilon}}}\right)\right\|_{L^{2}\left(\left[0, t^{*}\right], L_{x, v}^{2}\right)} \leqslant C, \\
& \left\|\frac{M_{f_{\varepsilon}}-f_{\varepsilon}}{\varepsilon^{4}}\left(1-\gamma\left(\frac{f_{\varepsilon}}{M_{f_{\varepsilon}}}\right)\right)\right\|_{L^{1}\left(\left[0, t^{*}\right], L_{x, v}^{1}\right)} \leqslant C .
\end{aligned}
$$

(ii) For all family $\left(\eta_{\varepsilon}\right)$ such that $\eta_{\varepsilon} \geqslant \varepsilon$,

$$
\frac{1}{\varepsilon^{2}} \frac{M_{f_{\varepsilon}}-f_{\varepsilon}}{M}=\mathrm{O}\left(\eta_{\varepsilon}\right)_{L_{\mathrm{loc}}^{1}\left(d t, L^{1}(d x M d v)\right)}+\mathrm{O}\left(\frac{1}{\eta_{\varepsilon}}\right)_{L_{t, x, v}^{\infty}} .
$$

Proof. - The first assertion is just a consequence of the dissipation bound (2.4), while the second one uses moreover the previous estimates on the fluctuation $g_{\varepsilon}$.

(i) As $r(z) \geqslant h(z)$ for all $z>-1$, we deduce from inequalities (3.1) and the properties (2.3) that

$$
\begin{aligned}
\frac{1}{\varepsilon^{4}} f_{\varepsilon} \frac{\left(M_{f_{\varepsilon}}-f_{\varepsilon}\right)^{2}}{f_{\varepsilon}^{2}} \gamma^{2}\left(\frac{f_{\varepsilon}}{M_{f_{\varepsilon}}}\right) & \leqslant \frac{C_{1}}{\varepsilon^{4}} f_{\varepsilon} h\left(\frac{M_{f_{\varepsilon}}}{f_{\varepsilon}}-1\right)\|\gamma\|_{\infty}^{2} \\
& \leqslant \frac{C_{1}}{\varepsilon^{4}} f_{\varepsilon} r\left(\frac{M_{f_{\varepsilon}}}{f_{\varepsilon}}-1\right)\|\gamma\|_{\infty}^{2}, \\
\frac{1}{\varepsilon^{4}}\left|M_{f_{\varepsilon}}-f_{\varepsilon}\right|\left|1-\gamma\left(\frac{f_{\varepsilon}}{M_{f_{\varepsilon}}}\right)\right| & \leqslant \frac{C_{1}}{\varepsilon^{4}} f_{\varepsilon} h\left(\frac{M_{f_{\varepsilon}}}{f_{\varepsilon}}-1\right)\|1-\gamma\|_{\infty} \\
& \leqslant \frac{C_{1}}{\varepsilon^{4}} f_{\varepsilon} r\left(\frac{M_{f_{\varepsilon}}}{f_{\varepsilon}}-1\right)\|1-\gamma\|_{\infty}
\end{aligned}
$$

which, coupled with (2.4), give the expected controls.

(ii) Apply Young's inequality (A.4) with $p=1, z=\frac{M_{f_{\varepsilon}}}{f_{\varepsilon}}-1$ and $\lambda=\varepsilon^{2} / \eta_{\varepsilon}$

$$
\begin{aligned}
\frac{1}{\varepsilon^{2}}\left|M_{f_{\varepsilon}}-f_{\varepsilon}\right| & \leqslant \frac{f_{\varepsilon}}{\varepsilon^{2}}\left(\frac{\eta_{\varepsilon}}{\varepsilon^{2}} r\left(\frac{M_{f_{\varepsilon}}}{f_{\varepsilon}}-1\right)+\frac{\varepsilon^{2}}{\eta_{\varepsilon}} h^{*}(1)\right) \\
& \leqslant \eta_{\varepsilon}\left(\frac{1}{\varepsilon^{4}} D\left(f_{\varepsilon}\right)+h^{*}(1) \frac{\varepsilon^{2}}{\eta_{\varepsilon}^{2}} \frac{1}{\varepsilon^{2}} f_{\varepsilon}\left(1-\gamma\left(\frac{f_{\varepsilon}}{M}\right)\right)\right)+\frac{h^{*}(1)}{\eta_{\varepsilon}} f_{\varepsilon} \gamma\left(\frac{f_{\varepsilon}}{M}\right) .
\end{aligned}
$$

As $\eta_{\varepsilon} \geqslant \varepsilon$, by (2.4) and Lemma 3.1, the first two terms are $\mathrm{O}\left(\eta_{\varepsilon}\right)$ in $L_{\mathrm{loc}}^{1}\left(d t, L_{x, v}^{1}\right)$. By (2.3), the last term is bounded by $\frac{3 h^{*}(1)\|\gamma\|_{\infty}}{2 \eta_{\varepsilon}} M$.

\subsection{Estimates on $M_{f_{\varepsilon}}-M$}

LEMMA 4.2. - Let $\left(g_{\varepsilon}^{0}\right)$ be a family of measurable functions on $\mathbb{R}^{d} \times \mathbb{R}^{d}$ satisfying (1.13) and (1.14). For each $\varepsilon>0$, consider a solution $f_{\varepsilon}=M\left(1+\varepsilon g_{\varepsilon}\right)$ of (1.2). Let $\chi_{\varepsilon}$ be the indicator 
function of $\left\{(t, x)|| R_{\varepsilon}-1\left|\leqslant \frac{1}{2},\right| U_{\varepsilon}\left|\leqslant \frac{1}{2},\right| T_{\varepsilon}-1 \mid \leqslant \frac{1}{2}\right\}$.

$$
\forall \alpha \in\left[0, \frac{2}{3}\left[, \quad \frac{\chi_{\varepsilon}}{\varepsilon} \frac{M_{f_{\varepsilon}}-M}{M^{\alpha}}=\mathrm{O}(1)_{L_{t}^{\infty}\left(L_{x}^{2}\left(L_{v}^{\infty}\right)\right)} .\right.\right.
$$

Proof. - Define the function $\mathcal{M}$ by

$$
\begin{aligned}
\mathcal{M}:(R, U, T, v) \in \mathbb{R}^{+} \times \mathbb{R}^{d} \times \mathbb{R}^{+} \times \mathbb{R}^{d} & \\
& \mapsto \mathcal{M}(R, U, T, v)=\frac{R}{(2 \pi T)^{d / 2}} \exp \left(-\frac{|v-U|^{2}}{2 T}\right)
\end{aligned}
$$

and denote by $K=\left[\frac{1}{2}, \frac{3}{2}\right] \times\left[-\frac{1}{2}, \frac{1}{2}\right]^{d} \times\left[\frac{1}{2}, \frac{3}{2}\right]$. Direct computations show that

$$
\begin{gathered}
\frac{D_{R} \mathcal{M}}{\mathcal{M}}(R, U, T, v)=\frac{1}{R}, \quad \frac{D_{U} \mathcal{M}}{\mathcal{M}}(R, U, T, v)=\frac{(v-U)}{T}, \\
\frac{D_{T} \mathcal{M}}{\mathcal{M}}(R, U, T, v)=-\frac{d}{2 T}+\frac{|v-U|^{2}}{2 T^{2}} .
\end{gathered}
$$

Then, as $T \leqslant \frac{3}{2}$ on $K$, for all $\alpha<\frac{2}{3}$, there exists $C_{\alpha}<+\infty$ such that

$$
\left\|\frac{D_{(R, U, T)} \mathcal{M}}{M^{\alpha}}\right\|_{L^{\infty}\left(K \times \mathbb{R}^{d}\right)} \leqslant C_{\alpha} .
$$

By Taylor's formula,

$$
\begin{aligned}
\chi_{\varepsilon} \frac{\left|M_{f_{\varepsilon}}-M\right|}{M^{\alpha}} & \leqslant\left\|\frac{D_{(R, U, T)} \mathcal{M}}{M^{\alpha}}\right\|_{L^{\infty}\left(K \times \mathbb{R}^{d}\right)} \chi_{\varepsilon}\left(\left|R_{\varepsilon}-1\right|+\left|U_{\varepsilon}\right|+\left|T_{\varepsilon}-1\right|\right) \\
& \leqslant C_{\alpha} \chi_{\varepsilon}\left(\left|R_{\varepsilon}-1\right|+\left|U_{\varepsilon}\right|+\left|T_{\varepsilon}-1\right|\right)
\end{aligned}
$$

for some $C_{\alpha}>0$. Lemma 3.2(ii) provides then the expected bound.

\subsection{Strengthened estimates on $f_{\varepsilon}-M$}

Combining Lemmas 4.1 and 4.2 allows to obtain some extra-integrability with respect to $v$ on the fluctuation $g_{\varepsilon}$.

LEMMA 4.3. - Let $\left(g_{\varepsilon}^{0}\right)$ be a family of measurable functions on $\mathbb{R}^{d} \times \mathbb{R}^{d}$ satisfying (1.13) and (1.14). For each $\varepsilon>0$, consider a solution $f_{\varepsilon}=M\left(1+\varepsilon g_{\varepsilon}\right)$ of (1.2). For all $\alpha \in\left[0, \frac{2}{3}[\right.$,

$$
\begin{aligned}
\frac{1}{\varepsilon^{2}} \frac{f_{\varepsilon}-M}{M^{\alpha}}= & \mathrm{O}\left(\frac{1}{|\log \varepsilon|}\right)_{L_{\mathrm{loc}}^{1}\left(d t, L^{1}\left(d x M^{\alpha} d v\right)\right)}+\mathrm{O}(|\log \varepsilon|)_{L_{t, x, v}^{\infty}} \\
& +\mathrm{O}\left(\frac{1}{\varepsilon}\right)_{L_{t}^{\infty}\left(L_{x}^{2}\left(L_{v}^{\infty}\right)\right)}+\mathrm{O}\left(\frac{1}{\varepsilon}\right)_{L_{t}^{\infty}\left(L_{x}^{1}\left(L_{v}^{\infty}\right)\right)} .
\end{aligned}
$$

Proof. - Let us first introduce the decomposition

$$
\begin{aligned}
\frac{1}{\varepsilon^{2}} \frac{f_{\varepsilon}-M}{M^{\alpha}}= & \frac{\left(1-\chi_{\varepsilon}\right)}{\varepsilon^{2}} \frac{f_{\varepsilon}-M}{M^{\alpha}} \mathbb{1}_{\frac{f_{\varepsilon}}{M}>\frac{1}{\varepsilon}}+\frac{\left(1-\chi_{\varepsilon}\right)}{\varepsilon^{2}} \frac{f_{\varepsilon}-M}{M^{\alpha}} \mathbb{1}_{\frac{f_{\varepsilon}}{M} \leqslant \frac{1}{\varepsilon}} \\
& +\frac{\chi_{\varepsilon}}{\varepsilon^{2}} \frac{f_{\varepsilon}-M_{f_{\varepsilon}}}{M^{\alpha}}+\frac{\chi_{\varepsilon}}{\varepsilon^{2}} \frac{M_{f_{\varepsilon}}-M}{M^{\alpha}}
\end{aligned}
$$


which is a refinement of decomposition (2.5). By Lemma 3.1(ii),

$$
\begin{aligned}
\left\|\frac{\left(1-\chi_{\varepsilon}\right)}{\varepsilon^{2}} \frac{f_{\varepsilon}-M}{M^{\alpha}} \mathbb{1}_{\frac{f_{\varepsilon}}{M}>\frac{1}{\varepsilon}}\right\|_{L^{\infty}\left(d t, L^{1}\left(d x M^{\alpha} d v\right)\right)} & \leqslant\left\|\frac{1}{\varepsilon^{2}}\left(f_{\varepsilon}-M\right) \mathbb{1}_{\frac{f_{\varepsilon}}{M}>\frac{1}{\varepsilon}}\right\|_{L_{t}^{\infty}\left(L_{x, v}^{1}\right)} \\
& \leqslant \frac{C}{|\log \varepsilon|}
\end{aligned}
$$

for some $C>0$, while by Lemma 3.2(ii)

$$
\left\|\frac{\left(1-\chi_{\varepsilon}\right)}{\varepsilon^{2}} \frac{f_{\varepsilon}-M}{M^{\alpha}} \mathbb{1}_{\frac{f_{\varepsilon}}{M} \leqslant \frac{1}{\varepsilon}}\right\|_{L_{t}^{\infty}\left(L_{x}^{1}\left(L_{v}^{\infty}\right)\right)} \leqslant\left\|\frac{\left(1-\chi_{\varepsilon}\right)}{\varepsilon^{3}} M^{1-\alpha}\right\|_{L_{t}^{\infty}\left(L_{x}^{1}\left(L_{v}^{\infty}\right)\right)} \leqslant \frac{C}{\varepsilon}
$$

for some $C>0$. Applying Lemma 4.1(ii) with $\eta_{\varepsilon}=|\log \varepsilon|^{-1}$ provides

$$
\frac{\chi_{\varepsilon}}{\varepsilon^{2}} \frac{M_{f_{\varepsilon}}-f_{\varepsilon}}{M^{\alpha}}=\mathrm{O}\left(\frac{1}{|\log \varepsilon|}\right)_{L_{\mathrm{loc}}^{1}\left(d t, L^{1}\left(d x M^{\alpha} d v\right)\right)}+\mathrm{O}(|\log \varepsilon|)_{L_{t, x, v}^{\infty}} .
$$

Finally, by Lemma 4.2,

$$
\left\|\frac{\chi_{\varepsilon}}{\varepsilon^{2}} \frac{M_{f_{\varepsilon}}-M}{M^{\alpha}}\right\|_{L_{t}^{\infty}\left(L_{x}^{2}\left(L_{v}^{\infty}\right)\right)} \leqslant \frac{C_{\alpha}}{\varepsilon}
$$

for some $C_{\alpha}>0$. Combining (4.3)-(4.7) leads to the expected estimate.

\section{5. $(x, v)$-interpolation estimates}

5.1. An auxiliary estimate on $\varepsilon^{-2}\left(1-\chi_{\varepsilon}\right)\left|f_{\varepsilon}-M\right|$

While the entropy-based estimates given in Lemma 3.2(ii) provide only a uniform bound on $\frac{1-\chi_{\varepsilon}}{\varepsilon^{2}}\left|f_{\varepsilon}-M\right|$ in $L_{t}^{\infty}\left(L_{x, v}^{1}\right)$, the strenghtened dissipation-based estimates stated in Lemma 4.3, coupled with the mixing properties of the operator $\left(\varepsilon \partial_{t}+v \cdot \nabla_{x}\right)$, imply that this same quantity actually converges to 0 as $\varepsilon \rightarrow 0$.

PROPOSITION 5.1. - Let $\left(g_{\varepsilon}^{0}\right)$ be a family of measurable functions on $\mathbb{R}^{d} \times \mathbb{R}^{d}$ satisfying (1.13) and (1.14). For each $\varepsilon>0$, consider a solution $f_{\varepsilon}=M\left(1+\varepsilon g_{\varepsilon}\right)$ of (1.2). Let $\alpha \in\left[0, \frac{2}{3}[\right.$ be a fixed constant. Define $\chi_{\varepsilon}$ as the indicator function of $\left\{(t, x)|| R_{\varepsilon}-1\left|\leqslant \frac{1}{2},\right| U_{\varepsilon} \mid \leqslant \frac{1}{2}\right.$, $\left.\left|T_{\varepsilon}-1\right| \leqslant \frac{1}{2}\right\}$. Then,

$$
\begin{aligned}
& \text { (i) } \frac{1-\chi_{\varepsilon}}{\varepsilon^{2}}\left(f_{\varepsilon}-M\right)=\mathrm{O}\left(\frac{1}{|\log \varepsilon|}\right)_{L_{\mathrm{loc}}^{1}\left(d t, L_{x, v}^{1}\right)}, \\
& \text { (ii) } \frac{1}{\varepsilon^{2}} \frac{f_{\varepsilon}-M}{M^{\alpha}}=\mathrm{O}\left(\frac{1}{|\log \varepsilon|}\right)_{L_{\mathrm{loc}}^{1}\left(d t, L^{1}\left(d x M^{\alpha} d v\right)\right)}+\mathrm{O}(|\log \varepsilon|)_{L_{t, x, v}^{\infty}}+\mathrm{O}\left(\frac{1}{\varepsilon}\right)_{L_{t}^{\infty}\left(L_{x}^{2}\left(L_{v}^{\infty}\right)\right)} \text {. }
\end{aligned}
$$

Proof. - Assertion (i) is obtained from Lemma 4.3 using mixing properties of the operator $\left(\varepsilon \partial_{t}+v \cdot \nabla_{x}\right)$, while assertion (ii) is a refinement of Lemma 4.3 coming directly from (i).

(i) Denote by $\phi=M^{\alpha}\left(1-\chi_{\varepsilon}\right) \geqslant 0$ for some fixed $\left.\alpha \in\right] 0, \frac{2}{3}$ [, and by $\Phi$ the solution of

$$
\partial_{\tau} \Phi+\left(\varepsilon \partial_{t}+v \cdot \nabla_{x}\right) \Phi=0
$$

such that $\Phi(\tau=0) \equiv \phi$. The maximum principle gives

$$
\left\|M^{-\alpha} \Phi\right\|_{L_{\tau, t, x, v}^{\infty}} \leqslant\left\|M^{-\alpha} \phi\right\|_{L_{t, x, v}^{\infty}} \leqslant 1 .
$$

$4^{\mathrm{e}}$ SÉRIE - TOME $36-2003-\mathrm{N}^{\circ} 2$ 
The following generalization of (2.7)

$$
\|\Phi(\sigma)\|_{L_{t}^{\infty}\left(L_{x}^{q}\left(L_{v}^{1}\right)\right)} \leqslant|\sigma|^{-d\left(1-\frac{1}{q}\right)}\|\phi\|_{L_{t}^{\infty}\left(L_{x}^{1}\left(L_{v}^{q}\right)\right)}
$$

coupled with Lemma 3.2(ii) provides, for all $1 \leqslant q \leqslant+\infty$,

$$
\|\Phi(\sigma)\|_{L_{t}^{\infty}\left(L_{x}^{q}\left(L_{v}^{1}\right)\right)} \leqslant C|\sigma|^{-d\left(1-\frac{1}{q}\right)} \varepsilon^{2} .
$$

Denote by $k=\varepsilon^{-2}\left|f_{\varepsilon}-M\right| / M^{\alpha}$. By Lemma 4.3,

$$
\begin{aligned}
k= & \mathrm{O}\left(\frac{1}{|\log \varepsilon|}\right)_{L_{\mathrm{loc}}^{1}\left(d t, L^{1}\left(d x M^{\alpha} d v\right)\right)}+\mathrm{O}(|\log \varepsilon|)_{L_{t, x, v}^{\infty}} \\
& +\mathrm{O}\left(\frac{1}{\varepsilon}\right)_{L_{t}^{\infty}\left(L_{x}^{2}\left(L_{v}^{\infty}\right)\right)}+\mathrm{O}\left(\frac{1}{\varepsilon}\right)_{L_{t}^{\infty}\left(L_{x}^{1}\left(L_{v}^{\infty}\right)\right)} .
\end{aligned}
$$

By (1.2),

$$
\left(\varepsilon \partial_{t}+v \cdot \nabla_{x}\right) k=\frac{1}{\varepsilon^{3}} \frac{M_{f_{\varepsilon}}-f_{\varepsilon}}{M^{\alpha}} \operatorname{sgn}\left(f_{\varepsilon}-M\right) .
$$

Then, by Lemma 4.1(ii) with $\eta_{\varepsilon}=\varepsilon$, we have

$$
\left(\varepsilon \partial_{t}+v \cdot \nabla_{x}\right) k=\mathrm{O}(1)_{L_{\mathrm{loc}}^{1}\left(d t, L^{1}\left(d x M^{\alpha} d v\right)\right)}+\mathrm{O}\left(\frac{1}{\varepsilon^{2}}\right)_{L_{t, x, v}^{\infty}} .
$$

Equipped with these a priori bounds, we deduce from Green's formula an estimate on $\frac{1-\chi_{\varepsilon}}{\varepsilon^{2}}\left|f_{\varepsilon}-M\right|=k \phi$

$$
\begin{aligned}
\iiint k \phi d x d v d t= & \int_{\varepsilon \tau}^{t^{*}} \iint_{0} k \Phi(\tau) d x d v d t \\
& -\int_{0}^{\tau} \int_{\varepsilon \sigma}^{t^{*}+\varepsilon \sigma} \iint \Phi(\sigma)\left(\varepsilon \partial_{t}+v \cdot \nabla_{x}\right) k d x d v d t d \sigma .
\end{aligned}
$$

Indeed, because of (5.5) and (5.2), (5.4), the first term in the right side of (5.7) satisfies

$$
\begin{aligned}
\left|\int_{\varepsilon \tau}^{t^{*}+\varepsilon \tau} \iint k \Phi(\tau) d x d v d t\right| \leqslant & \frac{C}{|\log \varepsilon|}\left\|M^{-\alpha} \Phi(\tau)\right\|_{L_{t, x, v}^{\infty}}+C t^{*}|\log \varepsilon|\|\Phi(\tau)\|_{L_{t}^{\infty}\left(L_{x, v}^{1}\right)} \\
& +\frac{C t^{*}}{\varepsilon}\|\Phi(\tau)\|_{L_{t}^{\infty}\left(L_{x}^{2}\left(L_{v}^{1}\right)\right)}+\frac{C t^{*}}{\varepsilon}\|\Phi(\tau)\|_{L_{t, x}^{\infty}\left(L_{v}^{1}\right)} \\
\leqslant & C\left(\frac{1}{|\log \varepsilon|}+\varepsilon^{2}|\log \varepsilon|+\varepsilon|\tau|^{-d / 2}+\varepsilon|\tau|^{-d}\right)
\end{aligned}
$$

while, because of (5.6) and (5.2), (5.4), the second term is estimated by

$$
\begin{aligned}
& \left|\int_{0}^{\tau} \int_{\varepsilon \sigma}^{t^{*}+\varepsilon \sigma} \iint \Phi(\sigma)\left(\varepsilon \partial_{t}+v \cdot \nabla_{x}\right) k d x d v d t d \sigma\right| \\
& \quad \leqslant C \tau\left(\left\|M^{-\alpha} \Phi\right\|_{L_{\tau, t, x, v}^{\infty}}+\frac{t^{*}}{\varepsilon^{2}}\|\Phi\|_{L_{\tau, t}^{\infty} L_{x, v}^{1}}\right) \leqslant C \tau .
\end{aligned}
$$


Then choosing $\tau=|\log \varepsilon|^{-1}$ in (5.8)-(5.9) and using identity (5.7) lead to

$$
\left\|\frac{1-\chi_{\varepsilon}}{\varepsilon^{2}}\left(f_{\varepsilon}-M\right)\right\|_{L^{1}\left(\left[0, t^{*}\right], L_{x, v}^{1}\right)}=\iint_{0}^{t^{*}} \iint k \phi d t d x d v \leqslant \frac{C}{|\log \varepsilon|} .
$$

(ii) A variant of decomposition (4.3) is the following

$$
\frac{1}{\varepsilon^{2}} \frac{f_{\varepsilon}-M}{M^{\alpha}}=\frac{\left(1-\chi_{\varepsilon}\right)}{\varepsilon^{2}} \frac{f_{\varepsilon}-M}{M^{\alpha}}+\frac{\chi_{\varepsilon}}{\varepsilon^{2}} \frac{f_{\varepsilon}-M_{f_{\varepsilon}}}{M^{\alpha}}+\frac{\chi_{\varepsilon}}{\varepsilon^{2}} \frac{M_{f_{\varepsilon}}-M}{M^{\alpha}} .
$$

By assertion (i),

$$
\frac{1-\chi_{\varepsilon}}{\varepsilon^{2}} \frac{f_{\varepsilon}-M}{M^{\alpha}}=\mathrm{O}\left(\frac{1}{|\log \varepsilon|}\right)_{L_{\mathrm{loc}}^{1}\left(d t, L^{1}\left(d x M^{\alpha} d v\right)\right)} .
$$

Then, estimating the two other terms by (4.6) and (4.7) gives assertion (ii).

\subsection{Convergence of ${ }^{\sharp} g_{\varepsilon}$ to 0}

This convergence result is very similar to the previous one, it uses a dual form of the classical dispersion estimates, and the refined estimates on the fluctuation $g_{\varepsilon}$ given in Proposition 5.1(ii). In order to establish it, we need the following preliminary results.

LEMMA 5.2. - Let $\left(g_{\varepsilon}^{0}\right)$ be a family of measurable functions on $\mathbb{R}^{d} \times \mathbb{R}^{d}$ satisfying (1.13) and (1.14). For each $\varepsilon>0$, consider a solution $f_{\varepsilon}=M\left(1+\varepsilon g_{\varepsilon}\right)$ of (1.2). Let $Q$ be any compact subset of $\mathbb{R}^{d}$, and $\gamma$ a bump function satisfying (2.3). Denote by

$$
\phi=\mathbb{1}_{x \in Q} M\left|1-\gamma\left(\frac{f_{\varepsilon}}{M}\right)\right|
$$

Then, for all $\alpha \in\left[\frac{1}{2}, 1\right]$, $\phi$ can be decomposed as $\phi_{1}+\phi_{2}$ with

(i)

$$
\begin{aligned}
& M^{-1} \phi_{j}=\mathrm{O}(1)_{L_{t, x, v}^{\infty}}, \phi_{j}=\mathrm{O}\left(\varepsilon^{2}\right)_{L_{t}^{\infty}\left(L_{x, v}^{1}\right)} \\
& \text { and } \forall \alpha^{\prime}<\alpha \leqslant 1, M^{-(1-\alpha)} \phi_{j}=\mathrm{O}\left(\varepsilon^{2 \alpha^{\prime}}\right)_{L_{t}^{\infty}\left(L_{x, v}^{1}\right)}
\end{aligned}
$$

(ii) $\forall \alpha^{\prime}<\alpha$,

$$
M^{-(1-\alpha)} \phi_{1}=\mathrm{O}\left(\varepsilon^{\alpha^{\prime}+1 / 2}\right)_{L_{t}^{\infty}\left(L_{x}^{1}\left(L_{v}^{2}\right)\right)} \quad \text { and } \quad M^{-(1-\alpha)} \phi_{2}=\mathrm{O}\left(\varepsilon^{\alpha^{\prime}+1 / 2}\right)_{L_{t}^{\infty}\left(L_{x}^{2}\left(L_{v}^{1}\right)\right)} .
$$

Proof. - We first establish a priori estimates on $\phi$ which $\phi_{1}$ and $\phi_{2}$ will inherit provided that $0 \leqslant \phi_{1}, \phi_{2} \leqslant \phi$. In a second step, we will introduce the convenient decomposition.

(i) By (2.3),

$$
\left\|M^{-1} \phi\right\|_{L_{t, x, v}^{\infty}} \leqslant\|1-\gamma\|_{\infty} \leqslant C
$$

while, by Lemma 3.1(i) and (iii),

$$
\|\phi\|_{L_{t}^{\infty}\left(L_{x, v}^{1}\right)} \leqslant C \varepsilon^{2} .
$$

Then, for all $\alpha^{\prime}<\alpha \leqslant 1$, 


$$
\begin{aligned}
\left\|M^{-(1-\alpha)} \phi\right\|_{L_{t}^{\infty}\left(L_{x, v}^{1}\right)} \leqslant & \left(\sup _{|v|^{2} \leqslant 4|\log \varepsilon|} M^{-(1-\alpha)}\right)\|\phi\|_{L_{t}^{\infty}\left(L_{x, v}^{1}\right)} \\
& +\left(\sup _{|v|^{2} \geqslant 4|\log \varepsilon|} M^{\alpha^{\prime}}\right)\left(\iint \mathbb{1}_{x \in Q} M^{\alpha-\alpha^{\prime}} d x d v\right)\left\|M^{-1} \phi\right\|_{L_{t, x, v}^{\infty}} \\
\leqslant & C \varepsilon^{-2(1-\alpha)}\|\phi\|_{L_{t}^{\infty} L_{x, v}^{1}}+C \varepsilon^{2 \alpha^{\prime}}\left\|M^{-1} \phi\right\|_{L_{t, x, v}^{\infty}}
\end{aligned}
$$

from which we deduce by (5.10) and (5.11) that

$$
\left\|M^{-(1-\alpha)} \phi\right\|_{L_{t}^{\infty}\left(L_{x, v}^{1}\right)} \leqslant C \varepsilon^{2 \alpha^{\prime}}
$$

for some $C>0$ depending only on $\alpha$ and $\alpha^{\prime}$.

(ii) Let $\alpha \leqslant 1$ be a fixed constant. Define

$$
\phi_{1}=\phi \mathbb{1}_{\int M^{-(1-\alpha)} \phi d v>\varepsilon}, \quad \phi_{2}=\phi \mathbb{1}_{\int M^{-(1-\alpha)} \phi d v \leqslant \varepsilon} .
$$

Then $\phi_{1}$ and $\phi_{2}$ satisfy estimates (5.9)-(5.11), as well as

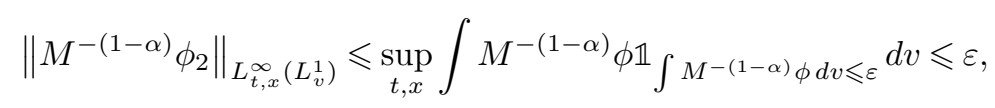

and, by (5.10), Chebyshev inequality and (5.12),

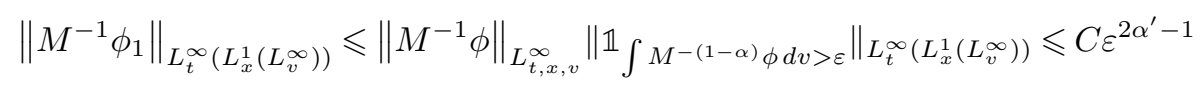

for all $\alpha^{\prime}<\alpha$. Then, combining (5.10)-(5.13) leads to

$$
\begin{aligned}
\left\|M^{-(1-\alpha)} \phi_{2}\right\|_{L_{t}^{\infty}\left(L_{x}^{2}\left(L_{v}^{1}\right)\right)} & \leqslant\left\|M^{-(1-\alpha)} \phi_{2}\right\|_{L_{t}^{\infty}\left(L_{x, v}^{1}\right)}^{1 / 2}\left\|M^{-(1-\alpha)} \phi_{2}\right\|_{L_{t}^{\infty}\left(L_{x}^{\infty}\left(L_{v}^{1}\right)\right)}^{1 / 2} \\
& \leqslant C \varepsilon^{\alpha^{\prime}} \varepsilon^{1 / 2}, \\
\left\|M^{-(1-\alpha)} \phi_{1}\right\|_{L_{t}^{\infty}\left(L_{x}^{1}\left(L_{v}^{2}\right)\right)} & \leqslant\left\|M^{\alpha-1 / 2}\right\|_{L_{v}^{\infty}}\left\|\phi_{1}\right\|_{L_{t}^{\infty}\left(L_{x, v}^{1}\right)}^{1 / 2}\left\|M^{-1} \phi_{1}\right\|_{L_{t}^{\infty}\left(L_{x}^{1}\left(L_{v}^{\infty}\right)\right)}^{1 / 2} \\
& \leqslant C \varepsilon \varepsilon^{\left(2 \alpha^{\prime}-1\right) / 2},
\end{aligned}
$$

for some $C>0$ depending on $\alpha$ and $\alpha^{\prime}$, and provided that $\alpha>1 / 2$.

PROPOSITION 5.3. - Let $\left(g_{\varepsilon}^{0}\right)$ be a family of measurable functions on $\mathbb{R}^{d} \times \mathbb{R}^{d}$ satisfying (1.13) and (1.14). For each $\varepsilon>0$, consider a solution $f_{\varepsilon}=M\left(1+\varepsilon g_{\varepsilon}\right)$ of (1.2), and define ${ }^{b} g_{\varepsilon}$ and ${ }^{\sharp} g_{\varepsilon}$ by (2.2) where $\gamma$ denotes any bump function satisfying (2.3). Let $\chi_{\varepsilon}$ be the indicator function of $\left\{(t, x)|| R_{\varepsilon}-1\left|\leqslant \frac{1}{2},\right| U_{\varepsilon}\left|\leqslant \frac{1}{2},\right| T_{\varepsilon}-1 \mid \leqslant \frac{1}{2}\right\}$. Then

(i) ${ }^{\sharp} g_{\varepsilon}=\mathrm{O}(1 /|\log \varepsilon|)_{L_{\mathrm{loc}}^{1}}\left(d t d x, L^{1}(M d v)\right)$,

(ii) $\forall p<+\infty,{ }^{\sharp} g_{\varepsilon} \chi_{\varepsilon}\left(1+|v|^{p}\right) \mathbb{1}_{|v|^{2} \leqslant|\log \varepsilon|^{2}}=\mathrm{o}(1)_{L_{\mathrm{loc}}^{1}\left(d t d x, L^{1}(M d v)\right)}$,

(iii) ${ }^{\sharp} g_{\varepsilon} \chi_{\varepsilon}\left(1+|v|^{2}\right)=\mathrm{o}(1)_{L_{\text {loc }}^{1}}\left(d t d x, L^{1}(M d v)\right)$.

Proof. - The first assertion is obtained from Proposition 5.1(ii) and Lemma 5.2 using again mixing properties of the operator $\left(\varepsilon \partial_{t}+v \cdot \nabla_{x}\right)$, while the two other assertions are deduced from the first one by interpolation.

(i) Let $Q$ be any compact subset of $\mathbb{R}^{d}$ and $\left.\alpha \in\right] \frac{1}{2}, \frac{2}{3}\left[\right.$ be a fixed number. Define $\phi, \phi_{1}$ and $\phi_{2}$ as in Lemma 5.2, and $\Phi_{1}$ as the solution of (5.1) with $\Phi_{1}(\tau=0) \equiv \phi_{1}$. By Lemma 5.2 and (5.3), for all $\alpha^{\prime}<\alpha$, 


$$
\begin{aligned}
& M^{-1} \phi_{2}=\mathrm{O}(1)_{L_{t, x, v}^{\infty}}, \quad M^{\alpha-1} \phi_{2}=\mathrm{O}\left(\varepsilon^{2 \alpha^{\prime}}\right)_{L_{t}^{\infty}\left(L_{x, v}^{1}\right)} \\
& M^{\alpha-1} \phi_{2}=\mathrm{O}\left(\varepsilon^{\alpha^{\prime}+1 / 2}\right)_{L_{t}^{\infty}\left(L_{x}^{2}\left(L_{v}^{1}\right)\right)}, \quad M^{-1} \Phi_{1}=\mathrm{O}(1)_{L_{\tau, t, x, v}^{\infty}}, \\
& M^{\alpha-1} \Phi_{1}=\mathrm{O}\left(\varepsilon^{2 \alpha^{\prime}}\right)_{L_{\tau, t}^{\infty}\left(L_{x, v}^{1}\right)}, \quad M^{\alpha-1} \Phi_{1}(\tau)=\mathrm{O}\left(|\tau|^{-\frac{d}{2}} \varepsilon^{\alpha^{\prime}+\frac{1}{2}}\right)_{L_{t}^{\infty}\left(L_{x}^{2}\left(L_{v}^{1}\right)\right)}
\end{aligned}
$$

Denote by $k=\varepsilon^{-2}\left|f_{\varepsilon}-M\right| / M$. By Proposition 5.1(ii),

$$
\begin{aligned}
k= & \mathrm{O}\left(\frac{1}{|\log \varepsilon|}\right)_{L_{\mathrm{loc}}^{1}\left(d t, L^{1}(d x M d v)\right)}+\mathrm{O}(|\log \varepsilon|)_{L^{\infty}\left(d t d x M^{1-\alpha} d v\right)} \\
& +\mathrm{O}\left(\frac{1}{\varepsilon}\right)_{L_{t}^{\infty}\left(L_{x}^{2}\left(L^{\infty}\left(M^{1-\alpha} d v\right)\right)\right)}
\end{aligned}
$$

and by (1.2) and Lemma 4.1(ii) with $\eta_{\varepsilon}=\varepsilon$,

$$
\left(\varepsilon \partial_{t}+v \cdot \nabla_{x}\right) k=\mathrm{O}(1)_{L_{\mathrm{loc}}^{1}\left(d t, L^{1}(d x M d v)\right)}+\mathrm{O}\left(\frac{1}{\varepsilon^{2}}\right)_{L_{t, x, v}^{\infty}}
$$

Then, in order to obtain an estimate on the quantity $\left.M\right|^{\sharp} g_{\varepsilon} \mid \mathbb{1}_{x \in Q}=k \phi$, we use essentially the same trick as in the proof of Proposition 5.1(i). Decompose $\int_{0}^{t^{*}} \iint k \phi d x d v d t$ according to Green's formula

$$
\begin{aligned}
\left.\iiint M\right|^{\sharp} g_{\varepsilon} \mid \mathbb{1}_{x \in Q} d x d v d t= & \int_{0}^{t^{*}} \iint k \phi_{2} d x d v d t+\int_{0}^{t^{*}} \iint k \phi_{1} d x d v d t \\
= & \iiint_{0}^{t^{*}} k \phi_{2} d x d v d t+\int_{\varepsilon \tau}^{t^{*}+\varepsilon \tau} \iint k \Phi_{1}(\tau) d x d v d t \\
& -\int_{0}^{\tau} \int_{\varepsilon \sigma}^{t^{*}+\varepsilon \sigma} \iint \Phi_{1}(\sigma)\left(\varepsilon \partial_{t}+v \cdot \nabla_{x}\right) k d x d v d t d \sigma .
\end{aligned}
$$

By (5.14) and (5.15), the first term in the right-side of (5.17) satisfies

$$
\begin{aligned}
& || \iint_{0}^{t^{*}} \iint_{2} d t d x d v \mid \\
& \quad \leqslant C\left(\frac{\left\|M^{-1} \phi_{2}\right\|_{L_{t, x, v}^{\infty}}}{|\log \varepsilon|}+t^{*}|\log \varepsilon|\left\|M^{\alpha-1} \phi_{2}\right\|_{L_{t}^{\infty}\left(L_{x, v}^{1}\right)}+\frac{t^{*}}{\varepsilon}\left\|M^{\alpha-1} \phi_{2}\right\|_{L_{t}^{\infty}\left(L_{x}^{2}\left(L_{v}^{1}\right)\right)}\right) \\
& \text { (5.18) } \leqslant C\left(\frac{1}{|\log \varepsilon|}+|\log \varepsilon| \varepsilon^{2 \alpha^{\prime}}+\varepsilon^{\alpha^{\prime}-\frac{1}{2}}\right)
\end{aligned}
$$

while the second term is estimated by

$$
\left|\int_{\varepsilon \tau}^{t^{*}+\varepsilon \tau} \iint k \Phi_{1}(\tau) d t d x d v\right| \leqslant C\left(\frac{1}{|\log \varepsilon|}+|\log \varepsilon| \varepsilon^{2 \alpha^{\prime}}+\varepsilon^{\alpha^{\prime}-\frac{1}{2}}|\tau|^{-\frac{d}{2}}\right)
$$

for some $C>0$ depending on $Q$ and $t^{*}$. From (5.14) and (5.16) we deduce that 


$$
\begin{aligned}
& \left|\int_{0}^{\tau} \int_{\varepsilon \sigma}^{t^{*}+\varepsilon \sigma} \iint \Phi_{1}(\sigma)\left(\varepsilon \partial_{t}+v \cdot \nabla_{x}\right) k d \sigma d t d x d v\right| \\
& \quad \leqslant C \tau\left(\left\|M^{-\alpha} \Phi_{1}\right\|_{L_{\tau, t, x, v}^{\infty}}+\frac{t^{*}}{\varepsilon^{2}}\left\|\Phi_{1}\right\|_{L_{\tau, t}^{\infty}\left(L_{x, v}^{1}\right)}\right) \leqslant C \tau
\end{aligned}
$$

holds for some $C>0$. Choose $\left.\alpha^{\prime} \in\right] \frac{1}{2}, \alpha\left[\right.$ and $\tau=|\log \varepsilon|^{-1}$ in (5.18)-(5.20) and plug these estimates in (5.17) to show that

$$
\left.\iiint M\right|^{\sharp} g_{\varepsilon} \mid \mathbb{1}_{x \in Q} d t d x d v \leqslant \frac{C}{|\log \varepsilon|}
$$

for some $C>0$ depending on $Q$ and $t^{*}$.

(ii) Let $Q$ be any compact subset of $\mathbb{R}^{d}$. From the previous bound we deduce that

$$
\left|{ }^{\sharp} g_{\varepsilon}\right|\left(1+|v|^{p}\right) \mathbb{1}_{|v|^{2} \leqslant 8 \log |\log \varepsilon|}=\mathrm{O}\left(\frac{(\log |\log \varepsilon|)^{p / 2}}{|\log \varepsilon|}\right)_{L^{1}\left(\left[0, t^{*}\right] \times Q, L^{1}(M d v)\right)} .
$$

In order to obtain a control on large velocities, we use the fact that $f_{\varepsilon}$ remains close to $M_{f_{\varepsilon}}$ which is known to have a good decay with respect to $v$ provided that the moments do not deviate too much from their mean values: Lemma 4.2 implies in particular that

$$
\forall \alpha<\frac{2}{3}, \exists C_{\alpha}>0, \quad \chi_{\varepsilon} M_{f_{\varepsilon}} \leqslant C_{\alpha} M^{\alpha} \text { uniformly in } \varepsilon .
$$

In order to use such a property, we rewrite the remainder $M^{\sharp} g_{\varepsilon}$ in terms of $M_{f_{\varepsilon}}$. Define $\Gamma: z \mapsto(z-1)(1-\gamma(z))$.

$$
\begin{aligned}
M^{\sharp} g_{\varepsilon} & =\frac{M}{\varepsilon^{2}} \Gamma\left(\frac{f_{\varepsilon}}{M}\right)=\frac{M}{\varepsilon^{2}} \Gamma\left(\frac{M_{f_{\varepsilon}}}{M}\right)+\frac{M}{\varepsilon^{2}}\left(\Gamma\left(\frac{f_{\varepsilon}}{M}\right)-\Gamma\left(\frac{M_{f_{\varepsilon}}}{M}\right)\right) \\
& =\frac{M}{\varepsilon^{2}} \Gamma\left(\frac{M_{f_{\varepsilon}}}{M}\right)-\frac{M}{\varepsilon^{2}} \int_{0}^{1} \Gamma^{\prime}\left(\frac{s M_{f_{\varepsilon}}+(1-s) f_{\varepsilon}}{M}\right) \frac{M_{f_{\varepsilon}}-f_{\varepsilon}}{M} d s
\end{aligned}
$$

from which we deduce that

$$
M^{\sharp} g_{\varepsilon}=\frac{M}{\varepsilon^{2}} \Gamma\left(\frac{M_{f_{\varepsilon}}}{M}\right)-\frac{M_{f_{\varepsilon}}-f_{\varepsilon}}{\varepsilon^{2}} \int_{0}^{1}(1-\tilde{\gamma})\left(\frac{s M_{f_{\varepsilon}}+(1-s) f_{\varepsilon}}{M}\right) d s
$$

where $\tilde{\gamma}: z \mapsto \gamma(z)+(z-1) \gamma^{\prime}(z)$.

From (2.3) we deduce that

$$
|\Gamma(z)|=|1-\gamma(z)||z-1| \leqslant 4|1-\gamma(z)||z-1|^{2} \leqslant 4\|1-\gamma\|_{\infty}|z-1|^{2} .
$$

Then, by Lemma 4.2, the first term in the right-side of (5.22) satisfies for any $\alpha \in] \frac{5}{8}, \frac{2}{3}[$

$$
\iint\left|\frac{M}{\varepsilon^{2}} \Gamma\left(\frac{M_{f_{\varepsilon}}}{M}\right)\right| \chi_{\varepsilon}\left(1+|v|^{p}\right) \mathbb{1}_{|v|^{2} \geqslant 8 \log |\log \varepsilon|} d v d x
$$




$$
\begin{aligned}
& \leqslant \frac{4\|1-\gamma\|_{\infty}}{\varepsilon^{2}} \int \frac{\left(M_{f_{\varepsilon}}-M\right)^{2}}{M} \chi_{\varepsilon}\left(1+|v|^{p}\right) \mathbb{1}_{|v|^{2} \geqslant 8 \log |\log \varepsilon|} d v \\
& \leqslant C_{\alpha} \int M^{2 \alpha-1}\left(1+|v|^{p}\right) \mathbb{1}_{|v|^{2} \geqslant 8 \log |\log \varepsilon|} d v \leqslant \frac{\widetilde{C}_{\alpha}}{|\log \varepsilon|}
\end{aligned}
$$

for some $C_{\alpha}, \widetilde{C}_{\alpha}>0$ depending on $\alpha$.

From (2.3) we also deduce that

$$
\|1-\tilde{\gamma}\|_{\infty} \leqslant\|1-\gamma\|_{\infty}+\frac{1}{2}\left\|\gamma^{\prime}\right\|_{\infty}<+\infty
$$

Then, in order to estimate the second term in the right-hand side of (5.22), decompose $q_{\varepsilon}=\left(f_{\varepsilon}-M_{f_{\varepsilon}}\right) / \varepsilon^{2} M_{f_{\varepsilon}}$ according to its size as in (2.5)

$$
\frac{f_{\varepsilon}-M_{f_{\varepsilon}}}{\varepsilon^{2} M_{f_{\varepsilon}}}=\frac{f_{\varepsilon}-M_{f_{\varepsilon}}}{\varepsilon^{2} M_{f_{\varepsilon}}} \gamma\left(\frac{f_{\varepsilon}}{M_{f_{\varepsilon}}}\right)+\frac{f_{\varepsilon}-M_{f_{\varepsilon}}}{\varepsilon^{2} M_{f_{\varepsilon}}}\left(1-\gamma\left(\frac{f_{\varepsilon}}{M_{f_{\varepsilon}}}\right)\right)={ }^{b} q_{\varepsilon}+\varepsilon^{2 \sharp} q_{\varepsilon} .
$$

By Lemma 4.1(i) and (2.3),

$$
\begin{aligned}
& \left\|M_{f_{\varepsilon}}{ }^{b} q_{\varepsilon}^{2}\right\|_{L^{1}\left(\left[0, t^{*}\right] \times \mathbb{R}^{2 d}\right)} \leqslant 2\left\|\frac{1}{\varepsilon^{2}} \frac{M_{f_{\varepsilon}}-f_{\varepsilon}}{f_{\varepsilon}{ }^{1 / 2}} \gamma\left(\frac{f_{\varepsilon}}{M_{f_{\varepsilon}}}\right)\right\|_{L^{2}\left(\left[0, t^{*}\right], L_{x, v}^{2}\right)}^{2} \leqslant C, \\
& \left\|M_{f_{\varepsilon}}^{\sharp} q_{\varepsilon}\right\|_{L^{1}\left(\left[0, t^{*}\right] \times \mathbb{R}^{2 d}\right)}=\left\|\frac{M_{f_{\varepsilon}}-f_{\varepsilon}}{\varepsilon^{4}}\left(1-\gamma\left(\frac{f_{\varepsilon}}{M_{f_{\varepsilon}}}\right)\right)\right\|_{L^{1}\left(\left[0, t^{*}\right], L_{x, v}^{1}\right)} \leqslant C,
\end{aligned}
$$

for some $C>0$. Then,

$$
\begin{aligned}
& \iint_{0}^{t^{*}} \iint_{f_{\varepsilon}} \frac{\left|M_{f_{\varepsilon}}\right|}{\varepsilon^{2}} \chi_{\varepsilon}\left(1+|v|^{p}\right) \mathbb{1}_{8 \log |\log \varepsilon| \leqslant|v|^{2} \leqslant|\log \varepsilon|^{2} \mathbb{1}_{x \in Q} d v d x d t} \\
& \leqslant\left.\iint_{0}^{t^{*}} \iint_{f_{\varepsilon}}\right|^{b} q_{\varepsilon} \mid \chi_{\varepsilon}\left(1+|v|^{p}\right) \mathbb{1}_{8 \log |\log \varepsilon| \leqslant|v|^{2} \mathbb{1}_{x \in Q} d v d x d t} \\
& \quad+\left.\varepsilon^{2} \iiint_{0}^{t^{*}} M_{f_{\varepsilon}}\right|^{\sharp} q_{\varepsilon} \mid\left(1+|v|^{p}\right) \mathbb{1}_{|v|^{2} \leqslant|\log \varepsilon|^{2}} d v d x d t \\
& \leqslant\left\|M_{f_{\varepsilon}}{ }^{b} q_{\varepsilon}^{2}\right\|_{L^{1}\left(\left[0, t^{*}\right] \times \mathbb{R}^{2 d}\right)}^{1 / 2} \\
& \quad \times\left(\int_{0}^{t^{*}} \iint_{f_{\varepsilon}} M_{\varepsilon}\left(1+|v|^{p}\right)^{2} \mathbb{1}_{|v|^{2} \geqslant 8 \log |\log \varepsilon|^{\mathbb{1}}} \chi_{x \in Q} d v d x d t\right)^{1 / 2} \\
& \quad+\varepsilon^{2}\left\|M_{f_{\varepsilon}}^{\sharp} q_{\varepsilon}\right\|_{L^{1}\left(\left[0, t^{*}\right] \times \mathbb{R}^{2 d}\right)}\left(1+|\log \varepsilon|^{p}\right) \\
& \leqslant C\left(C_{\alpha} t^{*}|Q| \int M^{\alpha}\left(1+|v|^{p}\right)^{2} \mathbb{1}_{|v|^{2} \geqslant 8 \log |\log \varepsilon|} d v\right)^{1 / 2}+C \varepsilon^{2}|\log \varepsilon|^{p} \leqslant \frac{\widetilde{C}_{\alpha}}{|\log \varepsilon|}
\end{aligned}
$$

for some $\widetilde{C}_{\alpha}>0$ depending on $\alpha, t^{*}$ and $|Q|$. Combining (5.22), (5.23) and (5.26) leads to 


$$
\left|{ }^{\sharp} g_{\varepsilon}\right|\left(1+|v|^{p}\right) \mathbb{1}_{8 \log |\log \varepsilon| \leqslant|v|^{2} \leqslant|\log \varepsilon|^{2}}=\mathrm{O}\left(\frac{1}{|\log \varepsilon|}\right)_{L_{\mathrm{loc}}^{1}\left(d t d x, L^{1}(M d v)\right)}
$$

which coupled with (5.21) gives the expected convergence.

(iii) Another way to estimate the second term in the right-hand side of (5.22) is to apply Young's inequality (A.4) with $p=\left(1+|v|^{2}\right) / 8, z=\left(f_{\varepsilon}-M_{f_{\varepsilon}}\right) / M_{f_{\varepsilon}}$ and $\lambda=\varepsilon^{2}|\log \varepsilon|^{1 / 2}$

$$
\begin{aligned}
\frac{1}{\varepsilon^{2}}\left|M_{f_{\varepsilon}}-f_{\varepsilon}\right|\left(1+|v|^{2}\right) & \leqslant \frac{8 M_{f_{\varepsilon}}}{\varepsilon^{2}}\left(\frac{1}{\varepsilon^{2}|\log \varepsilon|^{1 / 2}} r\left(\frac{f_{\varepsilon}-M_{f_{\varepsilon}}}{M_{f_{\varepsilon}}}\right)+\varepsilon^{2}|\log \varepsilon|^{1 / 2} h^{*}\left(\frac{1+|v|^{2}}{8}\right)\right) \\
& \leqslant \frac{8 M_{f_{\varepsilon}}}{\varepsilon^{2}}\left(\frac{1}{\varepsilon^{2}|\log \varepsilon|^{1 / 2}} r\left(\frac{f_{\varepsilon}-M_{f_{\varepsilon}}}{M_{f_{\varepsilon}}}\right)+\varepsilon^{2}|\log \varepsilon|^{1 / 2} e^{\left(1+|v|^{2}\right) / 8}\right)
\end{aligned}
$$

from which we deduce by (2.4) and Lemma 4.2 that for any $\alpha \in] \frac{5}{8}, \frac{2}{3}[$

$$
\begin{aligned}
& \iint_{0}^{t^{*}} \iint \frac{1}{\varepsilon^{2}}\left|M_{f_{\varepsilon}}-f_{\varepsilon}\right| \chi_{\varepsilon}\left(1+|v|^{2}\right) \mathbb{1}_{|v|^{2} \geqslant 8 \log |\log \varepsilon|} \mathbb{1}_{x \in Q} d v d x d t \\
& \leqslant \frac{8}{|\log \varepsilon|^{1 / 2}} \int_{0}^{t^{*}} \iint \frac{D\left(f_{\varepsilon}\right)}{\varepsilon^{4}} d x d v d t \\
& +8|\log \varepsilon|^{1 / 2} \iint_{0}^{t^{*}} \iint M_{f_{\varepsilon}} \chi_{\varepsilon} e^{\left(1+|v|^{2}\right) / 8} \mathbb{1}_{|v|^{2} \geqslant 8 \log |\log \varepsilon|} \mathbb{1}_{x \in Q} d v d x d t \\
& \leqslant \frac{8 C_{0}}{|\log \varepsilon|^{1 / 2}}+8 C_{\alpha}|\log \varepsilon|^{1 / 2} \iiint_{0}^{t^{*}} M^{\alpha} e^{\left(1+|v|^{2}\right) / 8} \mathbb{1}_{|v|^{2} \geqslant 8 \log |\log \varepsilon| \mathbb{1}_{x \in Q}} d v d x d t \\
& \leqslant \frac{\widetilde{C}_{\alpha}}{|\log \varepsilon|^{1 / 2}}
\end{aligned}
$$

for some $\widetilde{C}_{\alpha}>0$ depending on $\alpha, t^{*}$ and $|Q|$. Combining (5.22), (5.23), (5.26) and (5.21) allows to establish the last assertion.

\subsection{Weak compactness of $M\left({ }^{b} g_{\varepsilon}\right)^{2}$}

Proposition 5.4. - Let $\left(g_{\varepsilon}^{0}\right)$ be a family of measurable functions on $\mathbb{R}^{d} \times \mathbb{R}^{d}$ satisfying (1.13) and (1.14). For each $\varepsilon>0$, consider a solution $f_{\varepsilon}=M\left(1+\varepsilon g_{\varepsilon}\right)$ of (1.2), and define ${ }^{b} g_{\varepsilon}$ and ${ }^{\sharp} g_{\varepsilon}$ by (2.2) where $\gamma$ denotes any bump function satisfying (2.3). Then

(i) $M^{b} g_{\varepsilon}^{2}=\mathrm{O}(1 /|\log \varepsilon|)_{L_{\text {loc }}^{1}\left(d t, L_{x, v}^{1}\right)}+\mathrm{O}(\varepsilon|\log \varepsilon|)_{L_{t}^{\infty}\left(L_{x, v}^{2}\right)}+\mathrm{O}(1)_{L_{t}^{\infty}\left(L_{x}^{1}\left(L_{v}^{2}\right)\right)}$,

(ii) $\forall\left(\varepsilon_{n}\right)_{n \in \mathbb{N}}$ with $\varepsilon_{n} \rightarrow 0,\left(M^{b} g_{\varepsilon_{n}}^{2}\right)$ is relatively compact in $w-L_{\mathrm{loc}}^{1}\left(d t, L_{x, v}^{1}\right)$.

Proof. - Assertion (i) is obtained as a corollary of Proposition 5.1, while assertion (ii) is obtained from (i) by using the mixing properties of the free-transport operator $\left(\varepsilon \partial_{t}+v \cdot \nabla_{x}\right)$.

(i) Lemma 3.1 gives

$$
M^{1 / 2} g_{\varepsilon}=\mathrm{O}(1)_{L_{t}^{\infty}\left(L_{x, v}^{2}\right)}, \quad{ }^{b} g_{\varepsilon}=\mathrm{O}\left(\frac{1}{\varepsilon}\right)_{L_{t, x, v}^{\infty}} .
$$

As $\gamma$ is bounded in $L^{\infty}$, choosing $\alpha=\frac{1}{2}$ in Proposition 5.1(ii) leads to 


$$
\begin{aligned}
M^{1 / 2}{ }^{b} g_{\varepsilon} & =\frac{1}{\varepsilon} \frac{f_{\varepsilon}-M}{M^{1 / 2}} \gamma\left(\frac{f_{\varepsilon}}{M}\right) \\
& =\mathrm{O}\left(\frac{\varepsilon}{|\log \varepsilon|}\right)_{L_{\mathrm{loc}}^{1}\left(d t, L^{1}\left(d x M^{1 / 2} d v\right)\right)}+\mathrm{O}(\varepsilon|\log \varepsilon|)_{L_{t, x, v}^{\infty}}+\mathrm{O}(1)_{L_{t}^{\infty}\left(L_{x}^{2}\left(L_{v}^{\infty}\right)\right)} .
\end{aligned}
$$

Combining (5.27), (5.28) gives the expected estimate.

(ii) The estimate stated in assertion (i) shows that, up to a quantity that converges to 0 as $\varepsilon \rightarrow 0$, the family $\left(M^{b} g_{\varepsilon}^{2}\right)$ is essentially uniformly bounded in $L_{t}^{\infty}\left(L_{x}^{1}\left(L_{v}^{2}\right)\right)$, which means that any sequence $\left(M^{\mathrm{b}} g_{\varepsilon_{n}}^{2}\right)$ with $\varepsilon_{n} \rightarrow 0$ has extra-integrability with respect to $t$ and $v$. It remains to see that such a sequence is actually locally equiintegrable in all variables, using an interpolation argument as proposed in Section 2.4. Let $\Omega$ be any subset of $\left[0, t^{*}\right] \times \mathbb{R}^{d} \times \mathbb{R}^{d}$ of finite measure. Denote by $\phi$ the indicator function of $\Omega$, by $\phi_{0}, \phi_{1}$ and $\phi_{2}$ the following functions

$$
\begin{aligned}
& \phi_{0}=\phi \mathbb{1} \iint \phi d v d x>|\Omega|^{2 / 3}, \quad \phi_{1}=\left(\phi-\phi_{0}\right) \mathbb{1} \int\left(\phi-\phi_{0}\right) d v>|\Omega|^{1 / 3}, \\
& \phi_{2}=\left(\phi-\phi_{0}\right) \mathbb{1} \int\left(\phi-\phi_{0}\right) d v \leqslant|\Omega|^{1 / 3}
\end{aligned}
$$

and by $\Phi_{1}$ the solution of (5.1) with $\Phi_{1}(\tau=0)=\phi_{1}$. Using the same arguments as in the proof of Lemma 5.2, we obtain the following bounds: for all $j \in\{0,1,2\}$

$$
\left\|\phi_{j}\right\|_{L_{t, x, v}^{\infty}} \leqslant 1, \quad\left\|\phi_{j}\right\|_{L_{t, x, v}^{1}} \leqslant|\Omega|
$$

because $\phi_{j}$ is an indicator function,

$$
\begin{aligned}
& \left\|\phi-\phi_{0}\right\|_{L_{t}^{\infty}\left(L_{x, v}^{1}\right)} \leqslant|\Omega|^{2 / 3}, \quad\left\|\phi_{0}\right\|_{L_{t}^{1}\left(L_{x, v}^{\infty}\right)} \leqslant|\Omega|^{1 / 3}, \\
& \left\|\phi_{2}\right\|_{L_{t, x}^{\infty}\left(L_{v}^{1}\right)} \leqslant|\Omega|^{1 / 3}, \quad\left\|\phi_{1}\right\|_{L_{t}^{\infty}\left(L_{x}^{1}\left(L_{v}^{\infty}\right)\right)} \leqslant|\Omega|^{1 / 3},
\end{aligned}
$$

by Chebyshev inequality, and

$$
\left\|\Phi_{1}(\tau)\right\|_{L_{t, x}^{\infty}\left(L_{v}^{1}\right)} \leqslant|\Omega|^{1 / 3}|\tau|^{-d}
$$

by (5.3). Denote by $k=M^{b} g_{\varepsilon}^{2}$. By Lemma 3.1,

$$
k=\mathrm{O}(1)_{L_{t}^{\infty}\left(L_{x, v}^{1}\right)}
$$

while, by the assertion proved above,

$$
k=\mathrm{O}\left(\frac{1}{|\log \varepsilon|}\right)_{L_{\mathrm{loc}}^{1}\left(d t, L_{x, v}^{1}\right)}+\mathrm{O}(\varepsilon|\log \varepsilon|)_{L_{t}^{\infty} L_{x, v}^{2}}+\mathrm{O}(1)_{L_{t}^{\infty} L_{x}^{1} L_{v}^{2}}
$$

By (1.2),

$$
\left(\varepsilon \partial_{t}+v \cdot \nabla_{x}\right) k=\frac{2}{\varepsilon^{2}}\left(M_{f_{\varepsilon}}-f_{\varepsilon}\right) \tilde{\gamma}\left(\frac{f_{\varepsilon}}{M}\right)^{b} g_{\varepsilon}
$$

where $\tilde{\gamma}$ is defined as previously by $\tilde{\gamma}(z)=\gamma(z)+(z-1) \gamma^{\prime}(z)$. Applying Young's inequality (A.4) with $\lambda=1, z=\frac{M_{f_{\varepsilon}}}{f_{\varepsilon}}-1$ and $p=\varepsilon^{2 b} g_{\varepsilon} \tilde{\gamma}\left(\frac{f_{\varepsilon}}{M}\right)$ leads to

$$
\frac{1}{\varepsilon^{2}}\left|M_{f_{\varepsilon}}-f_{\varepsilon}\right|\left|\tilde{\gamma}\left(\frac{f_{\varepsilon}}{M}\right) g_{\varepsilon}\right| \leqslant \frac{f_{\varepsilon}}{\varepsilon^{4}}\left(r\left(\frac{M_{f_{\varepsilon}}}{f_{\varepsilon}}-1\right)+h^{*}\left(\left|\varepsilon^{2} \tilde{\gamma}\left(\frac{f_{\varepsilon}}{M}\right){ }^{b} g_{\varepsilon}\right|\right)\right) \text {. }
$$


Because $\left|\varepsilon^{b} g_{\varepsilon}\right| \leqslant 1$ and $\|\tilde{\gamma}\|_{\infty}<\infty$, the superquadratic homogeneity of $h^{*}$ stated in (A.3) provides for $\varepsilon$ sufficiently small,

$$
\begin{aligned}
\frac{1}{\varepsilon^{2}}\left|M_{f_{\varepsilon}}-f_{\varepsilon}\right|\left|\tilde{\gamma}\left(\frac{f_{\varepsilon}}{M}\right) b_{\varepsilon}\right| & \leqslant \frac{1}{\varepsilon^{4}} D\left(f_{\varepsilon}\right)+h^{*}(1) f_{\varepsilon} \tilde{\gamma}^{2}\left(\frac{f_{\varepsilon}}{M}\right) g_{\varepsilon}^{2} \\
& \leqslant \frac{1}{\varepsilon^{4}} D\left(f_{\varepsilon}\right)+\frac{3}{2} h^{*}(1)\|\tilde{\gamma}\|_{\infty}^{2} M^{b} g_{\varepsilon}^{2}
\end{aligned}
$$

from which we deduce that

$$
\left(\varepsilon \partial_{t}+v \cdot \nabla_{x}\right) k=\mathrm{O}(1)_{L_{\mathrm{loc}}^{1}\left(d t, L_{x, v}^{1}\right)} .
$$

Then, combine (5.29)-(5.34) and use Green's formula as in (5.17) to obtain the following estimate

$$
\begin{aligned}
\iiint_{\Omega} M^{\mathrm{b}} g_{\varepsilon}^{2} d v d x d t= & \iint_{0}^{t^{*}} \iint_{0} k \phi_{0} d v d x d t+\iiint_{0}^{t^{*}} k \phi_{2} d v d x d t+\int_{\varepsilon \tau}^{t^{*}+\varepsilon \tau} \iint_{0} k \Phi_{1}(\tau) d v d x d t \\
& -\int_{0}^{\tau} \int_{\varepsilon \sigma}^{t^{*}+\varepsilon \sigma} \iint_{1}(\sigma)\left(\varepsilon \partial_{t}+v \cdot \nabla_{x}\right) k d \sigma d v d x d t \\
= & \mathrm{O}\left(|\Omega|^{1 / 3}\right)+\left(\mathrm{O}\left(\frac{1}{|\log \varepsilon|}\right)+\mathrm{O}\left(\varepsilon|\log \varepsilon||\Omega|^{1 / 2}\right)+\mathrm{O}\left(|\Omega|^{1 / 6}\right)\right) \\
& +\left(\mathrm{O}\left(\frac{1}{|\log \varepsilon|}\right)+\mathrm{O}\left(\varepsilon|\log \varepsilon||\Omega|^{1 / 2}\right)+\mathrm{O}\left(|\Omega|^{1 / 6}|\tau|^{-d / 2}\right)\right)+\mathrm{O}(|\tau|)
\end{aligned}
$$

Choosing $|\tau|=|\Omega|^{1 / 6 d}$ shows that any subsequence of $\left(M^{b} g_{\varepsilon}^{2}\right)$ is locally equiintegrable in $L^{1}\left(\left[0, t^{*}\right] \times \mathbb{R}^{d} \times \mathbb{R}^{d}\right)$, which implies the expected weak compactness by Dunford Pettis theorem.

Propositions 5.3 and 5.4 imply together Theorem 2.2. They further give some control on large velocities, which will be useful to establish the convergence of the conservation defects (Section 6). In order to take limits in the fluxes (Section 7), we will also need some strong compactness on the hydrodynamic fields $\left\langle{ }^{b} g_{\varepsilon}\right\rangle,\left\langle{ }^{b} g_{\varepsilon} v\right\rangle$ and $\left\langle{ }^{b} g_{\varepsilon}|v|^{2}\right\rangle$. Velocity averaging lemmas provide some regularity with respect to the variable $x$ on the moments of ${ }^{b} g_{\varepsilon}$, from which we can deduce that the non-oscillating parts of the moments are strongly compact in $L_{\text {loc }}^{2}\left(\mathbb{R}^{+} \times \mathbb{R}^{d}\right)$ (see (7.26)). Moreover, this extra regularity in $x$ allows to get a rigorous description of the acoustic waves, i.e. of the oscillating parts of the moments (see (7.29)).

COROLlARY 5.5. - Let $\left(g_{\varepsilon}^{0}\right)$ be a family of measurable functions on $\mathbb{R}^{d} \times \mathbb{R}^{d}$ satisfying (1.13) and (1.14). For each $\varepsilon>0$, consider a solution $f_{\varepsilon}=M\left(1+\varepsilon g_{\varepsilon}\right)$ of (1.2), and define ${ }^{b} g_{\varepsilon}$ and ${ }^{\sharp} g_{\varepsilon}$ by (2.2) where $\gamma$ denotes any bump function satisfying (2.3). Consider a sequence $\left(\varepsilon_{n}\right)$ with $\varepsilon_{n} \rightarrow 0$. Then, for each function $\psi \equiv \psi(v)$ in $L^{2}(M d v)$, each $t^{*}>0$ and each compact $Q \subset \mathbb{R}^{d}$, there exists a function $\eta$ in $C\left(\mathbb{R}_{+}\right)$such that

$$
\left\|\int{ }^{b} g_{\varepsilon_{n}}(t, x+\delta y, v) \psi(v) M(v) d v-\int{ }^{b} g_{\varepsilon_{n}}(t, x, v) \psi(v) M(v) d v\right\|_{L^{2}\left(\left[0, t^{*}\right] \times Q\right)} \leqslant \eta(\delta)
$$

for each $\delta>0, y \in B(0,1)$, uniformly in $n \in \mathbb{N}$. 
Proof. - In order to establish this last a priori estimate, we just have to check that the sequence ${ }^{b} g_{\varepsilon_{n}}$ satisfies the assumptions of the velocity averaging result stated in Proposition B.1. By Lemma 3.1(i), for all $\varepsilon>0,{ }^{b} g_{\varepsilon}$ belongs to $L_{\text {loc }}^{2}\left(d t, L^{2}(d x M d v)\right)$.

By Proposition 5.4, the sequence ${ }^{b} g_{\varepsilon_{n}}^{2}$ is relatively compact in $w-L_{\mathrm{loc}}^{1}\left(d t d x, L^{1}(M d v)\right)$.

Let $t^{*}$ be a nonnegative constant, $Q$ be a compact subset of $\mathbb{R}^{d}$, and $\Omega$ be some subset of $\left[0, t^{*}\right] \times Q \times \mathbb{R}^{d}$. By (1.2),

$$
\left(\varepsilon \partial_{t}+v \cdot \nabla_{x}\right) M^{b} g_{\varepsilon}=\frac{2}{\varepsilon^{2}}\left(M_{f_{\varepsilon}}-f_{\varepsilon}\right) \tilde{\gamma}\left(\frac{f_{\varepsilon}}{M}\right)
$$

where $\tilde{\gamma}$ is defined as previously.Young's inequality (A.4) provides, for all $\eta \geqslant \varepsilon^{2}$,

$$
\begin{aligned}
& \int_{0}^{t^{*}} \iint_{Q}\left|\frac{1}{\varepsilon^{2}}\left(M_{f_{\varepsilon}}-f_{\varepsilon}\right) \tilde{\gamma}\left(\frac{f_{\varepsilon}}{M}\right)\right| d v d x d t \\
& \leqslant \frac{\eta}{\varepsilon^{4}} \int_{0}^{t^{*}} \iint_{Q} D\left(f_{\varepsilon}\right) d v d x d t+\frac{3\|\tilde{\gamma}\|_{\infty}^{2}}{2 \eta} \iiint_{\Omega} M d v d x d t
\end{aligned}
$$

from which we deduce that $\left(\varepsilon \partial_{t}+v \cdot \nabla_{x}\right) M^{b} g_{\varepsilon_{n}}$ is locally equiintegrable, and thus compact in $w-L^{1}\left(\left[0, t^{*}\right] \times Q \times \mathbb{R}^{d}\right)$.

\section{The chain-rule for ${ }^{b} g_{\varepsilon}$}

Let $\left(g_{\varepsilon}^{0}\right)$ be a family of measurable functions on $\mathbb{R}^{d} \times \mathbb{R}^{d}$ satisfying (1.13) and (1.14). For each $\varepsilon>0$, consider a solution $f_{\varepsilon}=M\left(1+\varepsilon g_{\varepsilon}\right)$ of (1.2), and define ${ }^{b} g_{\varepsilon}$ and ${ }^{\sharp} g_{\varepsilon}$ by (2.2) where $\gamma$ denotes any bump function satisfying (2.3). Denote by $\xi(v)$ either 1 , or $v_{i}$ for some $i \in\{1,2, \ldots, d\}$, or $|v|^{2}$. From (1.2), we deduce that

$$
\partial_{t}\left\langle{ }^{b} g_{\varepsilon} \xi(v)\right\rangle+\frac{1}{\varepsilon} \nabla_{x} \cdot\left\langle{ }^{b} g_{\varepsilon} \xi(v) v\right\rangle=\frac{1}{\varepsilon^{3}} \int\left(M_{f_{\varepsilon}}-f_{\varepsilon}\right) \tilde{\gamma}\left(\frac{f_{\varepsilon}}{M}\right) \xi(v) d v
$$

where $\tilde{\gamma}$ is defined by

$$
\tilde{\gamma}(z)=(z-1) \gamma^{\prime}(z)+\gamma(z)
$$

and thus satisfies properties (2.3).

By definition of $M_{f_{\varepsilon}}, \int M_{f_{\varepsilon}} \xi(v) d v=\int f_{\varepsilon} \xi(v) d v$. Then, the conservation defects satisfy

$$
\frac{1}{\varepsilon^{3}} \int\left(M_{f_{\varepsilon}}-f_{\varepsilon}\right) \tilde{\gamma}\left(\frac{f_{\varepsilon}}{M}\right) \xi(v) d v=\frac{1}{\varepsilon^{3}} \int\left(M_{f_{\varepsilon}}-f_{\varepsilon}\right)\left(\tilde{\gamma}\left(\frac{f_{\varepsilon}}{M}\right)-1\right) \xi(v) d v
$$

If $\xi(v)=1$, the estimates on $\left(M_{f_{\varepsilon}}-f_{\varepsilon}\right)$ and on ${ }^{\sharp} g_{\varepsilon}$ established in Sections 3-5 allow to prove that the conservation defect converges to 0 as $\varepsilon \rightarrow 0$. In the other cases, because of the contribution of large velocities, we need refined estimates in order to establish such a convergence. The key idea is that the decay of $M_{f_{\varepsilon}}$ with respect to $v$ depends only on the values of the moments $R_{\varepsilon}$, $U_{\varepsilon}$ and $T_{\varepsilon}$.

The proof of Theorem 2.3 is then based on the following identity 


$$
\begin{aligned}
\partial_{t}\left\langle{ }^{b} g_{\varepsilon} \xi(v)\right\rangle+\frac{1}{\varepsilon} \nabla_{x} \cdot\left\langle{ }^{b} g_{\varepsilon} \xi(v) v\right\rangle= & \frac{1-\tilde{\chi}_{\varepsilon}}{\varepsilon^{3}} \int\left(M_{f_{\varepsilon}}-f_{\varepsilon}\right) \tilde{\gamma}\left(\frac{f_{\varepsilon}}{M}\right) \xi(v) d v \\
& +\frac{\tilde{\chi}_{\varepsilon}}{\varepsilon^{3}} \int\left(M_{f_{\varepsilon}}-f_{\varepsilon}\right)\left(\tilde{\gamma}\left(\frac{f_{\varepsilon}}{M}\right)-1\right) \xi(v) d v
\end{aligned}
$$

where $\tilde{\chi}_{\varepsilon}$ is a convenient macroscopic truncation, such that $1-\tilde{\chi}_{\varepsilon}=\mathrm{o}\left(\varepsilon^{2}\right)_{L_{\mathrm{loc}}^{1}(d t d x)}$ and $\tilde{\chi}_{\varepsilon} M_{f_{\varepsilon}}$ has a good decay in $v$.

\subsection{Introducing a new macroscopic truncation}

Proposition 6.1. - Let $\left(g_{\varepsilon}^{0}\right)$ be a family of measurable functions on $\mathbb{R}^{d} \times \mathbb{R}^{d}$ satisfying (1.13) and (1.14). For each $\varepsilon>0$, consider a solution $f_{\varepsilon}=M\left(1+\varepsilon g_{\varepsilon}\right)$ of (1.2). Define $\tilde{\chi}_{\varepsilon}$ as the indicator function of $\left\{(t, x)|| R_{\varepsilon}-\left.1\left|\leqslant \frac{1}{2},\right| U_{\varepsilon}\left|\leqslant \frac{1}{2},\right| \log \varepsilon\right|^{-1} \leqslant T_{\varepsilon} \leqslant \log |\log \varepsilon|\right\}$. Then,

$$
\frac{1-\tilde{\chi}_{\varepsilon}}{\varepsilon^{2}}=\mathrm{O}\left(\frac{1}{\log |\log \varepsilon|}\right)_{L_{\mathrm{loc}}^{1}\left(d t, L_{x}^{1}\right)} .
$$

Proof. - By Proposition 5.1,

$$
\frac{1}{\varepsilon^{2}} \int_{0}^{t^{*}} \int\left(1-\chi_{\varepsilon}\right)\left|R_{\varepsilon}-1\right| d x d t \leqslant \frac{1}{\varepsilon^{2}} \int_{0}^{t^{*}} \iint\left(1-\chi_{\varepsilon}\right)\left|f_{\varepsilon}-M\right| d v d x d t \leqslant \frac{C}{|\log \varepsilon|}
$$

and

$$
\begin{aligned}
\frac{1}{\varepsilon^{2}} \int_{0}^{t^{*}} \int\left(1-\chi_{\varepsilon}\right)\left|R_{\varepsilon} U_{\varepsilon}\right| d x d t & \leqslant \frac{1}{\varepsilon^{2}} \int_{0}^{t^{*}} \iint_{0}\left(1-\chi_{\varepsilon}\right)\left|f_{\varepsilon}-M\right||v| d v d x d t \\
\leqslant & \left(\frac{1}{\varepsilon^{2}} \iiint_{0}^{t^{*}}\left(1-\chi_{\varepsilon}\right)\left|f_{\varepsilon}-M\right| d v d x d t\right)^{1 / 2} \\
& \times\left(\frac{1}{\varepsilon^{2}} \iiint\left(1-\chi_{\varepsilon}\right)\left|f_{\varepsilon}-M\right||v|^{2} d v d x d t\right)^{1 / 2} \\
& \leqslant \frac{C}{|\log \varepsilon|^{1 / 2}}
\end{aligned}
$$

using the second estimate in Lemma 3.2(ii) to control the second factor in the right-hand side of (6.4). From (6.3) we deduce that

$$
\frac{1}{\varepsilon^{2}} \int_{0}^{t^{*}} \int_{\left|R_{\varepsilon}-1\right| \geqslant 1 / 2} d x d t=\mathrm{O}\left(\frac{1}{|\log \varepsilon|}\right)
$$

while (6.4) implies

$$
\frac{1}{\varepsilon^{2}} \int_{0}^{t^{*}} \int_{\left|R_{\varepsilon}-1\right| \leqslant 1 / 2} \mathbb{1}_{\left|U_{\varepsilon}\right| \geqslant 1 / 2} d x d t=\mathrm{O}\left(\frac{1}{|\log \varepsilon|^{1 / 2}}\right) .
$$


If the temperature satisfies $T_{\varepsilon} \leqslant|\log \varepsilon|^{-1}$ or $T_{\varepsilon} \geqslant \log |\log \varepsilon|$, for $\varepsilon$ sufficiently small, we have $k\left(T_{\varepsilon}-1\right)=T_{\varepsilon}-\log T_{\varepsilon}-1 \geqslant \frac{1}{2} \log |\log \varepsilon|$. Then, by Lemma 3.2(i),

$$
\begin{aligned}
& \frac{1}{\varepsilon^{2}} \int_{0}^{t^{*}} \int_{\left|R_{\varepsilon}-1\right| \leqslant 1 / 2}\left(\mathbb{1}_{T_{\varepsilon} \leqslant|\log \varepsilon|^{-1}}+\mathbb{1}_{T_{\varepsilon} \geqslant \log |\log \varepsilon|}\right) d x d t \\
& \quad \leqslant \frac{4}{\varepsilon^{2}} \int_{0}^{t^{*}} \int_{\varepsilon} \frac{k\left(T_{\varepsilon}-1\right)}{\log |\log \varepsilon|} d x d t \leqslant \frac{C}{\log |\log \varepsilon|}
\end{aligned}
$$

for some $C>0$. Combining (6.5)-(6.7) gives the expected estimate.

COROLlARY 6.2. - Let $\left(g_{\varepsilon}^{0}\right)$ be a family of measurable functions on $\mathbb{R}^{d} \times \mathbb{R}^{d}$ satisfying (1.13) and (1.14). For each $\varepsilon>0$, consider a solution $f_{\varepsilon}=M\left(1+\varepsilon g_{\varepsilon}\right)$ of (1.2). Define $\hat{\gamma}$ by (6.1) where $\gamma$ denotes any bump function satisfying (2.3), and $\tilde{\chi}_{\varepsilon}$ as the indicator function of $\left\{(t, x)|| R_{\varepsilon}-\left.1\left|\leqslant \frac{1}{2},\right| U_{\varepsilon}\left|\leqslant \frac{1}{2},\right| \log \varepsilon\right|^{-1} \leqslant T_{\varepsilon} \leqslant \log |\log \varepsilon|\right\}$. Then,

$$
\frac{1-\tilde{\chi}_{\varepsilon}}{\varepsilon^{3}} \int\left(M_{f_{\varepsilon}}-f_{\varepsilon}\right) \tilde{\gamma}\left(\frac{f_{\varepsilon}}{M}\right) \xi(v) d v \rightarrow 0 \quad \text { in } L_{\mathrm{loc}}^{1}(d t d x)
$$

Proof. - It is easy to check that the function $\tilde{\gamma}$ defined by (6.1) satisfies properties (2.3). Then, applying Young's inequality (A.4) with

$$
p=|\xi(v)| / 4, \quad z=\left(M_{f_{\varepsilon}}-f_{\varepsilon}\right) / f_{\varepsilon} \quad \text { and } \quad \lambda=\varepsilon \sqrt{\log |\log \varepsilon|} \leqslant 1
$$

leads to

$$
\begin{aligned}
& \frac{1-\tilde{\chi}_{\varepsilon}}{\varepsilon^{3}}\left|\left(M_{f_{\varepsilon}}-f_{\varepsilon}\right) \tilde{\gamma}\left(\frac{f_{\varepsilon}}{M}\right) \xi(v)\right| \\
& \leqslant \frac{4\left(1-\tilde{\chi}_{\varepsilon}\right)}{\varepsilon^{3}} f_{\varepsilon}\left|\tilde{\gamma}\left(\frac{f_{\varepsilon}}{M}\right)\right| \\
& \quad \times\left(\frac{1}{\varepsilon \sqrt{\log |\log \varepsilon|}} r\left(\frac{M_{f_{\varepsilon}}-f_{\varepsilon}}{f_{\varepsilon}}\right)+\varepsilon \sqrt{\log |\log \varepsilon|} h^{*}\left(\frac{|\xi(v)|}{4}\right)\right) \\
& \leqslant \frac{4\|\tilde{\gamma}\|_{\infty}}{\sqrt{\log |\log \varepsilon|}} \frac{D\left(f_{\varepsilon}\right)}{\varepsilon^{4}}+6 \sqrt{\log |\log \varepsilon|}\|\tilde{\gamma}\|_{\infty} \frac{\left(1-\tilde{\chi}_{\varepsilon}\right)}{\varepsilon^{2}} M h^{*}\left(\frac{|\xi(v)|}{4}\right) .
\end{aligned}
$$

Using (2.4) and Proposition 6.1 shows that the right-side of (6.8) converges to 0 in $L_{\mathrm{loc}}^{1}\left(d t d x, L_{v}^{1}\right)$.

\subsection{Establishing decay estimates on Maxwellian distributions}

LEMma 6.3. - Fix $p \in \mathbb{N}$. Let $\left(g_{\varepsilon}^{0}\right)$ be a family of measurable functions on $\mathbb{R}^{d} \times \mathbb{R}^{d}$ satisfying (1.13) and (1.14). For each $\varepsilon>0$, consider a solution $f_{\varepsilon}=M\left(1+\varepsilon g_{\varepsilon}\right)$ of (1.2). Define $\tilde{\chi}_{\varepsilon}$ as the indicator function of $\left\{(t, x)|| R_{\varepsilon}-\left.1\left|\leqslant \frac{1}{2},\right| U_{\varepsilon}\left|\leqslant \frac{1}{2},\right| \log \varepsilon\right|^{-1} \leqslant T_{\varepsilon} \leqslant \log |\log \varepsilon|\right\}$. Then

(i) $\tilde{\chi}_{\varepsilon} M_{f_{\varepsilon}}|v|^{p} \mathbb{1}_{|v|^{2} \geqslant(\log |\log \varepsilon|)^{2}}=\mathrm{o}(1)_{L_{t, x}^{\infty}\left(L_{v}^{1}\right)}$,

(ii) $\forall p<+\infty, \tilde{\chi}_{\varepsilon} M_{f_{\varepsilon}} h^{*}\left(\frac{|\xi(v)|}{4 \log |\log \varepsilon|}\right) \mathbb{1}_{|v|^{2} \geqslant|\log \varepsilon|^{2}}=\mathrm{o}\left(\varepsilon^{p}\right)_{L_{t, x}^{\infty}\left(L_{v}^{1}\right)}$. 
Proof. - In order to establish Lemma 6.3, we use classical estimates on Maxwellian distributions:

$$
\int_{\lambda}^{+\infty} e^{-r^{2} / 2} r^{q} d r \sim_{\lambda \rightarrow+\infty} e^{-\lambda^{2} / 2} \lambda^{q-1} .
$$

(i) By definition of $\tilde{\chi}_{\varepsilon}$, the following inequalities hold: $\tilde{\chi}_{\varepsilon} R_{\varepsilon} \leqslant 3 / 2, \tilde{\chi}_{\varepsilon}\left|U_{\varepsilon}\right| \leqslant 1$ and $\tilde{\chi}_{\varepsilon} T_{\varepsilon} \leqslant \log |\log \varepsilon|$. Then, define the variable $r=\left|v-U_{\varepsilon}\right| / \sqrt{T_{\varepsilon}}$. For almost all $t, x$,

$$
\begin{aligned}
& \int \tilde{\chi}_{\varepsilon} M_{f_{\varepsilon}}|v|^{p} \mathbb{1}_{|v|^{2} \geqslant(\log |\log \varepsilon|)^{2}} d v \\
& \leqslant \int \tilde{\chi}_{\varepsilon} M_{f_{\varepsilon}}\left(\left|v-U_{\varepsilon}\right|+1\right)^{p} \mathbb{1}_{\left|v-U_{\varepsilon}\right|^{2} \geqslant \frac{1}{2}(\log |\log \varepsilon|)^{2}} d v \\
& \leqslant \frac{1}{(2 \pi)^{d / 2}}\left|S^{d-1}\right| \tilde{\chi}_{\varepsilon} R_{\varepsilon}\left(T_{\varepsilon}^{1 / 2}+1\right)^{p} \int_{\sqrt{\frac{1}{2} \log |\log \varepsilon|}}^{\infty} \exp \left(-\frac{r^{2}}{2}\right)(r+1)^{p} r^{d-1} d r \\
& \leqslant \frac{C_{p}(\log |\log \varepsilon|)^{p+d / 2-1}}{|\log \varepsilon|^{1 / 4}}
\end{aligned}
$$

for some $C_{p}>0$ depending on $p$.

(ii) Fix $p<+\infty$. For $\varepsilon$ sufficiently small, the same method as previously gives for almost all $t, x$,

$$
\begin{aligned}
& \int \tilde{\chi}_{\varepsilon} M_{f_{\varepsilon}} h^{*}\left(\frac{|\xi(v)|}{4 \log |\log \varepsilon|}\right) \mathbb{1}_{|v|^{2} \geqslant|\log \varepsilon|^{2}} d v \\
& \leqslant \int \tilde{\chi}_{\varepsilon} M_{f_{\varepsilon}} \exp \left(\frac{\left|v-U_{\varepsilon}\right|^{2}+2}{4 \log |\log \varepsilon|}\right) \mathbb{1}_{|v|^{2} \geqslant|\log \varepsilon|^{2}} d v \\
& \leqslant e^{1 / 2} \int \tilde{\chi}_{\varepsilon} M_{f_{\varepsilon}} \exp \left(\frac{\left|v-U_{\varepsilon}\right|^{2}}{4 T_{\varepsilon}}\right) \mathbb{1}_{\left|v-U_{\varepsilon}\right|^{2} \geqslant 5 p|\log \varepsilon| T_{\varepsilon}} d v \\
& \leqslant e^{1 / 2} \int \tilde{\chi}_{\varepsilon} \frac{R_{\varepsilon}}{\left(2 \pi T_{\varepsilon}\right)^{d / 2}} \exp \left(-\frac{\left|v-U_{\varepsilon}\right|^{2}}{4 T_{\varepsilon}}\right) \mathbb{1}_{\left|v-U_{\varepsilon}\right|^{2} \geqslant 5 p|\log \varepsilon| T_{\varepsilon}} d v \\
& \leqslant \frac{3}{2} e^{1 / 2} \frac{\left|S^{d-1}\right|}{(2 \pi)^{d / 2}} \int_{\sqrt{5 p|\log \varepsilon|}}^{\infty} \exp \left(-\frac{r^{2}}{4}\right) r^{d-1} d r \leqslant C_{p} \varepsilon^{5 p / 4}|\log \varepsilon|^{d / 2-1}
\end{aligned}
$$

for some $C_{p}>0$ depending on $p$.

COROLlARY 6.4. - Let $\left(g_{\varepsilon}^{0}\right)$ be a family of measurable functions on $\mathbb{R}^{d} \times \mathbb{R}^{d}$ satisfying (1.13) and (1.14). For each $\varepsilon>0$, consider a solution $f_{\varepsilon}=M\left(1+\varepsilon g_{\varepsilon}\right)$ of (1.2), and define ${ }^{b} g_{\varepsilon}$ and ${ }^{\sharp} g_{\varepsilon}$ by (2.2) where $\gamma$ denotes any bump function satisfying (2.3). Let $\tilde{\chi}_{\varepsilon}$ be the indicator function of $\left\{(t, x)|| R_{\varepsilon}-\left.1\left|\leqslant \frac{1}{2},\right| U_{\varepsilon}\left|\leqslant \frac{1}{2},\right| \log \varepsilon\right|^{-1} \leqslant T_{\varepsilon} \leqslant \log |\log \varepsilon|\right\}$. Then,

$$
\forall p<+\infty, \quad{ }^{\sharp} g_{\varepsilon} \tilde{\chi}_{\varepsilon}\left(1+|v|^{p}\right) \mathbb{1}_{|v| \leqslant|\log \varepsilon|}=\mathrm{o}(1)_{L_{\mathrm{loc}}^{1}\left(d t d x, L^{1}(M d v)\right)} .
$$

Proof. - This estimate is obtained from Proposition 5.3 and Lemma 6.3 by using the following decomposition

$$
\begin{aligned}
& { }^{\sharp} g_{\varepsilon} \tilde{\chi}_{\varepsilon}\left(1+|v|^{p}\right) \mathbb{1}_{|v| \leqslant|\log \varepsilon|} \\
& \quad={ }^{\sharp} g_{\varepsilon} \chi_{\varepsilon}\left(1+|v|^{p}\right) \mathbb{1}_{|v| \leqslant|\log \varepsilon|}+{ }^{\sharp} g_{\varepsilon} \tilde{\chi}_{\varepsilon}\left(1-\chi_{\varepsilon}\right)\left(1+|v|^{p}\right) \mathbb{1}_{|v| \leqslant|\log \varepsilon|}
\end{aligned}
$$


where $\chi_{\varepsilon}$ denotes the indicator function of $\left\{(t, x)|| R_{\varepsilon}-1\left|\leqslant \frac{1}{2},\right| U_{\varepsilon}\left|\leqslant \frac{1}{2},\right| T_{\varepsilon}-1 \mid \leqslant \frac{1}{2}\right\}$. By Proposition 5.3(ii), the first term satisfies

$$
{ }^{\sharp} g_{\varepsilon} \chi_{\varepsilon}\left(1+|v|^{p}\right) \mathbb{1}_{|v| \leqslant|\log \varepsilon|}=\mathrm{o}(1)_{L_{\mathrm{loc}}^{1}\left(d t d x, L^{1}(M d v)\right)} .
$$

In order to estimate the second term in the right-side of (6.9), we introduce a new decomposition based on the velocity tail

$$
\begin{aligned}
\left.M\right|^{\sharp} g_{\varepsilon} \mid \tilde{\chi}_{\varepsilon}\left(1-\chi_{\varepsilon}\right)\left(1+|v|^{p}\right) \mathbb{1}_{|v| \leqslant|\log \varepsilon|} & =\left.M\right|^{\sharp} g_{\varepsilon} \mid \tilde{\chi}_{\varepsilon}\left(1-\chi_{\varepsilon}\right)\left(1+|v|^{p}\right) \mathbb{1}_{|v| \leqslant \log |\log \varepsilon|} \\
& +\left.M\right|^{\sharp} g_{\varepsilon} \mid \tilde{\chi}_{\varepsilon}\left(1-\chi_{\varepsilon}\right)\left(1+|v|^{p}\right) \mathbb{1}_{\log |\log \varepsilon|<|v| \leqslant|\log \varepsilon|} \\
\leqslant & \left.C M\right|^{\sharp} g_{\varepsilon} \mid(\log |\log \varepsilon|)^{p} \\
& +\frac{C}{\varepsilon^{2}}\left(f_{\varepsilon}-M_{f_{\varepsilon}}+M_{f_{\varepsilon}}+M\right) \tilde{\chi}_{\varepsilon}\left(1-\chi_{\varepsilon}\right)\left(1+|v|^{p}\right) \mathbb{1}_{\log |\log \varepsilon|<|v| \leqslant|\log \varepsilon|}
\end{aligned}
$$

By Proposition 5.3(i),

$$
{ }^{\sharp} g_{\varepsilon}(\log |\log \varepsilon|)^{p}=\mathrm{o}(1)_{L_{\mathrm{loc}}^{1}\left(d t d x, L^{1}(M d v)\right)} .
$$

Decompose $\frac{1}{\varepsilon^{2}}\left(f_{\varepsilon}-M_{f_{\varepsilon}}\right)=M_{f_{\varepsilon}}\left({ }^{b} q_{\varepsilon}+\varepsilon^{2 \sharp} q_{\varepsilon}\right)$ as in (2.5) where ${ }^{b} q_{\varepsilon}$ and ${ }^{\sharp} q_{\varepsilon}$ satisfy (5.24). Then,

$$
\begin{aligned}
& \left\|\frac{1}{\varepsilon^{2}}\left(f_{\varepsilon}-M_{f_{\varepsilon}}\right) \tilde{\chi}_{\varepsilon}\left(1+|v|^{p}\right) \mathbb{1}_{\log |\log \varepsilon| \leqslant|v| \leqslant|\log \varepsilon|}\right\|_{L^{1}\left(\left[0, t^{*}\right] \times Q \times \mathbb{R}^{d}\right)} \\
& \leqslant\left\|M_{f_{\varepsilon}}{ }^{b} q_{\varepsilon} \tilde{\chi}_{\varepsilon}\left(1+|v|^{p}\right) \mathbb{1}_{\log |\log \varepsilon|<|v|}\right\|_{L^{1}\left(\left[0, t^{*}\right] \times Q \times \mathbb{R}^{d}\right)} \\
& \quad+\varepsilon^{2}\left\|M_{f_{\varepsilon}}^{\sharp} q_{\varepsilon} \tilde{\chi}_{\varepsilon}\left(1+|v|^{p}\right) \mathbb{1}_{|v| \leqslant|\log \varepsilon|}\right\|_{L^{1}\left(\left[0, t^{*}\right] \times Q \times \mathbb{R}^{d}\right)} \\
& \leqslant\left\|M_{f_{\varepsilon}}{ }^{b} q_{\varepsilon}^{2}\right\|_{L^{1}\left(\left[0, t^{*}\right] \times \mathbb{R}^{2 d}\right)}^{1 / 2}\left\|M_{f_{\varepsilon}} \tilde{\chi}_{\varepsilon}\left(1+|v|^{p}\right)^{2} \mathbb{1}_{\log |\log \varepsilon|<|v|}\right\|_{L^{1}\left(\left[0, t^{*}\right] \times Q \times \mathbb{R}^{d}\right)}^{1 / 2} \\
& \quad+\varepsilon^{2}\left\|M_{f_{\varepsilon}}^{\sharp} q_{\varepsilon}\right\|_{L^{1}\left(\left[0, t^{*}\right] \times \mathbb{R}^{2 d}\right)}\left\|\left(1+|v|^{p}\right) \mathbb{1}_{|v| \leqslant|\log \varepsilon|}\right\|_{L^{\infty}\left(\mathbb{R}^{d}\right)}
\end{aligned}
$$

from which we deduce by (5.24) and Lemma 6.3(i) that

$$
\frac{1}{\varepsilon^{2}}\left(f_{\varepsilon}-M_{f_{\varepsilon}}\right) \tilde{\chi}_{\varepsilon}\left(1+|v|^{p}\right) \mathbb{1}_{\log |\log \varepsilon|<|v|^{2} \leqslant|\log \varepsilon|^{2}}=\mathrm{o}(1)_{L_{\mathrm{loc}}^{1}\left(d t d x, L_{v}^{1}\right)} .
$$

By Lemma 3.2(ii) and Lemma 6.3(i),

$$
\frac{\left(1-\chi_{\varepsilon}\right)}{\varepsilon^{2}} M_{f_{\varepsilon}} \tilde{\chi}_{\varepsilon}\left(1+|v|^{p}\right) \mathbb{1}_{\log |\log \varepsilon|<|v| \leqslant|\log \varepsilon|}=\mathrm{o}(1)_{L_{\mathrm{loc}}^{1}}\left(d t d x, L_{v}^{1}\right)
$$

and

$$
\frac{\left(1-\chi_{\varepsilon}\right)}{\varepsilon^{2}} M \tilde{\chi}_{\varepsilon}\left(1+|v|^{p}\right) \mathbb{1}_{\log |\log \varepsilon|<|v| \leqslant|\log \varepsilon|}=\mathrm{o}(1)_{L_{\operatorname{loc}}^{1}\left(d t d x, L_{v}^{1}\right)} .
$$

Combining (6.11)-(6.15) shows that

$$
{ }^{\sharp} g_{\varepsilon} \tilde{\chi}_{\varepsilon}\left(1-\chi_{\varepsilon}\right)\left(1+|v|^{p}\right) \mathbb{1}_{|v| \leqslant|\log \varepsilon|}=\mathrm{o}(1)_{L_{\mathrm{loc}}^{1}\left(d t d x, L^{1}(M d v)\right)}
$$

which, coupled with (6.9), (6.10), gives the expected estimate. 


\subsection{Estimating the conservation defects}

By Corollary 6.2, the first term in the right-side of (6.2) converges to 0 in $L_{\mathrm{loc}}^{1}(d t d x)$. It remains then to estimate the second term

$$
\begin{aligned}
& \frac{\tilde{\chi}_{\varepsilon}}{\varepsilon^{3}} \int\left(M_{f_{\varepsilon}}-f_{\varepsilon}\right)\left(\tilde{\gamma}\left(\frac{f_{\varepsilon}}{M}\right)-1\right) \xi(v) d v \\
& =\frac{\tilde{\chi}_{\varepsilon}}{\varepsilon^{3}} \int\left(M_{f_{\varepsilon}}-f_{\varepsilon}\right)\left(\tilde{\gamma}\left(\frac{f_{\varepsilon}}{M}\right)-1\right) \xi(v) \mathbb{1}_{|v|^{2}>|\log \varepsilon|^{2}} d v \\
& \quad+\frac{\tilde{\chi}_{\varepsilon}}{\varepsilon^{3}} \int\left(M_{f_{\varepsilon}}-f_{\varepsilon}\right)\left(\tilde{\gamma}\left(\frac{f_{\varepsilon}}{M}\right)-1\right) \xi(v) \mathbb{1}_{|v|^{2} \leqslant|\log \varepsilon|^{2}} d v
\end{aligned}
$$

where $\xi(v)$ denotes any collision invariant (i.e. either 1 , or $v_{i}$ for some $i \in\{1,2, \ldots, d\}$ or $|v|^{2}$ ). Apply Young's inequality (A.4) with $p=|\xi(v)| /(4 \log |\log \varepsilon|), z=\left(f_{\varepsilon}-M_{f_{\varepsilon}}\right) / M_{f_{\varepsilon}}$ and $\lambda=1$ and use (2.4) and Lemma 6.3(ii) to estimate the first term in the right-side of (6.17)

$$
\begin{aligned}
& \frac{\tilde{\chi}_{\varepsilon}}{\varepsilon^{3}} \int\left|M_{f_{\varepsilon}}-f_{\varepsilon}\right||\xi(v)| \mathbb{1}_{|v|^{2} \geqslant|\log \varepsilon|^{2}} d v \\
& \quad \leqslant \frac{4 \log |\log \varepsilon|}{\varepsilon^{3}} \int D\left(f_{\varepsilon}\right) d v+\frac{4 \tilde{\chi}_{\varepsilon} \log |\log \varepsilon|}{\varepsilon^{3}} \int M_{f_{\varepsilon}} h^{*}\left(\frac{|\xi(v)|}{4 \log |\log \varepsilon|}\right) \mathbb{1}_{|v|^{2} \geqslant|\log \varepsilon|^{2}} d v
\end{aligned}
$$

from which we deduce that

$$
\frac{\tilde{\chi}_{\varepsilon}}{\varepsilon^{3}} \int\left(M_{f_{\varepsilon}}-f_{\varepsilon}\right)\left(\tilde{\gamma}\left(\frac{f_{\varepsilon}}{M}\right)-1\right) \xi(v) \mathbb{1}_{|v|^{2} \geqslant|\log \varepsilon|^{2}} d v=\mathrm{o}(1)_{L_{\mathrm{loc}}^{1}(d t d x)} .
$$

In order to control the part with moderate velocities, we use the decomposition given in Lemma 4.1(i),

$$
\begin{aligned}
& \frac{\tilde{\chi}_{\varepsilon}}{\varepsilon^{3}} \int_{0}^{t^{*}} \iint_{Q}\left|\left(M_{f_{\varepsilon}}-f_{\varepsilon}\right)\left(\tilde{\gamma}\left(\frac{f_{\varepsilon}}{M}\right)-1\right) \xi(v)\right| \mathbb{1}_{|v|^{2} \leqslant|\log \varepsilon|^{2}} d v d x d t \\
& \leqslant \frac{\tilde{\chi}_{\varepsilon}}{\varepsilon} \iint_{0}^{t^{*}} \iint_{Q}\left|\frac{\left(M_{f_{\varepsilon}}-f_{\varepsilon}\right)}{\varepsilon^{2}} \gamma\left(\frac{f_{\varepsilon}}{M_{f_{\varepsilon}}}\right)\left(\tilde{\gamma}\left(\frac{f_{\varepsilon}}{M}\right)-1\right) \xi(v)\right| \mathbb{1}_{|v|^{2} \leqslant|\log \varepsilon|^{2}} d v d x d t \\
& +\varepsilon \iint_{0}^{t^{*}} \iint_{Q}\left|\frac{\left(M_{f_{\varepsilon}}-f_{\varepsilon}\right)}{\varepsilon^{4}}\left(1-\gamma\left(\frac{f_{\varepsilon}}{M_{f_{\varepsilon}}}\right)\right)\left(\tilde{\gamma}\left(\frac{f_{\varepsilon}}{M}\right)-1\right) \xi(v)\right| \mathbb{1}_{|v|^{2} \leqslant|\log \varepsilon|^{2}} d v d x d t \\
& \leqslant\left\|\frac{1}{\varepsilon^{2}} \frac{M_{f_{\varepsilon}}-f_{\varepsilon}}{f_{\varepsilon}^{1 / 2}} \gamma\left(\frac{f_{\varepsilon}}{M_{f_{\varepsilon}}}\right)\right\|_{L^{2}\left(\left[0, t^{*}\right], L_{x, v}^{2}\right)} \\
& \times\left\|\frac{\tilde{\chi}_{\varepsilon}}{\varepsilon} f_{\varepsilon}^{1 / 2}\left(\tilde{\gamma}\left(\frac{f_{\varepsilon}}{M}\right)-1\right) \xi(v) \mathbb{1}_{|v|^{2} \leqslant|\log \varepsilon|^{2}}\right\|_{L^{2}\left(\left[0, t^{*}\right] \times Q \times \mathbb{R}^{d}\right)} \\
& +\varepsilon\left\|\frac{M_{f_{\varepsilon}}-f_{\varepsilon}}{\varepsilon^{4}}\left(1-\gamma\left(\frac{f_{\varepsilon}}{M_{f_{\varepsilon}}}\right)\right)\right\|_{L^{1}\left(\left[0, t^{*}\right], L_{x, v}^{1}\right)}\|1-\tilde{\gamma}\|_{\infty}
\end{aligned}
$$


As $\tilde{\gamma}$ satisfies properties (2.3), by Corollary 6.4,

$$
\frac{1}{\varepsilon^{2}}\left|f_{\varepsilon}-M\right|\left|1-\tilde{\gamma}\left(\frac{f_{\varepsilon}}{M}\right)\right| \tilde{\chi}_{\varepsilon}\left(1+|v|^{4}\right) \mathbb{1}_{|v| \leqslant|\log \varepsilon|}=\mathrm{o}(1)_{L_{\mathrm{loc}}^{1}\left(d t d x, L_{v}^{1}\right)} .
$$

Moreover, by Lemma 3.1(iii),

$$
\begin{aligned}
\frac{1}{\varepsilon^{2}} f_{\varepsilon}\left(\tilde{\gamma}\left(\frac{f_{\varepsilon}}{M}\right)-1\right)^{2} & \leqslant \frac{1}{\varepsilon^{2}} f_{\varepsilon}\left|\tilde{\gamma}\left(\frac{f_{\varepsilon}}{M}\right)-1\right|\|1-\tilde{\gamma}\|_{\infty} \\
& \leqslant 9\|1-\tilde{\gamma}\|_{\infty} \frac{\left|f_{\varepsilon}-M\right|}{\varepsilon^{2}}\left|1-\tilde{\gamma}\left(\frac{f_{\varepsilon}}{M}\right)\right| .
\end{aligned}
$$

Combining (6.20) and (6.21) and the first control in Lemma 4.1(i) shows that the first term in the right-side of (6.19) converges to 0

$$
\begin{aligned}
& \left\|\frac{1}{\varepsilon^{2}} \frac{M_{f_{\varepsilon}}-f_{\varepsilon}}{f_{\varepsilon}^{1 / 2}} \gamma\left(\frac{f_{\varepsilon}}{M_{f_{\varepsilon}}}\right)\right\|_{L^{2}\left(\left[0, t^{*}\right], L_{x, v}^{2}\right)} \\
& \quad \times\left\|\frac{\tilde{\chi}_{\varepsilon}}{\varepsilon} f_{\varepsilon}^{1 / 2}\left(\tilde{\gamma}\left(\frac{f_{\varepsilon}}{M}\right)-1\right) \xi(v) \mathbb{1}_{|v|^{2} \leqslant|\log \varepsilon|^{2}}\right\|_{L^{2}\left(\left[0, t^{*}\right], L_{x, v}^{2}\right)} \rightarrow 0 .
\end{aligned}
$$

The second control in Lemma 4.1(i) shows that the second term in the right-side of (6.19) converges to 0

$$
\begin{aligned}
& \varepsilon\left\|\frac{M_{f_{\varepsilon}}-f_{\varepsilon}}{\varepsilon^{4}}\left(1-\gamma\left(\frac{f_{\varepsilon}}{M_{f_{\varepsilon}}}\right)\right)\right\|_{L^{1}\left(\left[0, t^{*}\right], L_{x, v}^{1}\right)} \\
& \quad \times\|1-\tilde{\gamma}\|_{\infty}\left\|\xi(v) \mathbb{1}_{|v|^{2} \leqslant|\log \varepsilon|^{2}}\right\|_{L^{\infty}\left(\mathbb{R}^{d}\right)} \rightarrow 0 .
\end{aligned}
$$

Combining (6.19), (6.22) and (6.23) gives

$$
\frac{\tilde{\chi}_{\varepsilon}}{\varepsilon^{3}} \int\left|\left(M_{f_{\varepsilon}}-f_{\varepsilon}\right)\left(\tilde{\gamma}\left(\frac{f_{\varepsilon}}{M}\right)-1\right) \xi(v)\right| \mathbb{1}_{|v|^{2} \leqslant|\log \varepsilon|^{2}} d v=\mathrm{o}(1)_{L_{\mathrm{loc}}^{1}}(d t d x)
$$

which, coupled with (6.17) and (6.18) shows that the second term in the right-side of (6.2) converges to 0 in $L_{\text {loc }}^{1}(d t d x)$. Theorem 2.3 is then established.

\section{Derivation of the incompressible Navier-Stokes equations}

\subsection{The limiting BGK equation}

Equipped with the a priori estimates established in Sections 3-5, we can establish the limiting form of the fluctuation $g_{\varepsilon}$ and of $\varepsilon^{-2}\left(M_{f_{\varepsilon}}-f_{\varepsilon}\right)$.

LEMMA 7.1. - Let $\left(g_{\varepsilon}^{0}\right)$ be a family of measurable functions on $\mathbb{R}^{d} \times \mathbb{R}^{d}$ satisfying (1.13) and (1.14). For each $\varepsilon>0$, consider a solution $f_{\varepsilon}=M\left(1+\varepsilon g_{\varepsilon}\right)$ of (1.2), and define ${ }^{b} g_{\varepsilon}$ by (2.2) where $\gamma$ denotes any bump function satisfying (2.3). Any limit point of the family $\left({ }^{b} g_{\varepsilon}\right)$ is a function $g \in L^{\infty}\left(d t, L^{2}(d x M d v)\right)$ such that

$$
g(t, x, v)=\rho(t, x)+u(t, x) \cdot v+\theta(t, x) \frac{|v|^{2}-d}{2} .
$$


Proof. - By Lemma 3.1(i), the family $\left({ }^{b} g_{\varepsilon}\right)$ is compact in $w-L^{\infty}\left(d t, w-L^{2}(d x M d v)\right)$. Let $g$ be any of its limit points and consider a sequence $\left(\varepsilon_{n}\right)$ such that $\varepsilon_{n} \rightarrow 0$ and ${ }^{b} g_{\varepsilon_{n}} \rightarrow g$ in $w-L^{\infty}\left(d t, L^{2}(d x M d v)\right)$. Define $\chi_{\varepsilon}$ as in Lemma 3.2. Then,

$$
\begin{aligned}
& M {\left[\frac{\chi_{\varepsilon}}{\varepsilon}\left(\left(R_{\varepsilon}-1\right)+U_{\varepsilon} \cdot v+\left(T_{\varepsilon}-1\right) \frac{|v|^{2}-d}{2}\right)-{ }^{b} g_{\varepsilon}\right] } \\
&= M \frac{\chi_{\varepsilon}}{\varepsilon}\left[\left(\left(R_{\varepsilon}-1\right)+U_{\varepsilon} \cdot v+\left(T_{\varepsilon}-1\right) \frac{|v|^{2}-d}{2}\right)-\varepsilon g_{\varepsilon}\right]+\varepsilon M \chi_{\varepsilon}^{\sharp} g_{\varepsilon}-M\left(1-\chi_{\varepsilon}\right)^{b} g_{\varepsilon} \\
&= \frac{\chi_{\varepsilon}}{\varepsilon}\left[M\left(\left(R_{\varepsilon}-1\right)+U_{\varepsilon} \cdot v+\left(T_{\varepsilon}-1\right) \frac{|v|^{2}-d}{2}\right)+M-M_{f_{\varepsilon}}\right]+\frac{\chi_{\varepsilon}}{\varepsilon}\left(M_{f_{\varepsilon}}-f_{\varepsilon}\right) \\
& \text { (7.2) } \quad+\varepsilon M \chi_{\varepsilon}^{\sharp} g_{\varepsilon}-M\left(1-\chi_{\varepsilon}\right)^{b} g_{\varepsilon} .
\end{aligned}
$$

By Lemma 4.1(ii) with $\eta_{\varepsilon}=1$, the second term in the right-side of (7.2) converges to zero in $L_{\mathrm{loc}}^{1}\left(d t d x, L_{v}^{1}\right)$

$$
\frac{\chi_{\varepsilon}}{\varepsilon}\left(M_{f_{\varepsilon}}-f_{\varepsilon}\right)=\mathrm{O}(\varepsilon)_{L_{\mathrm{loc}}^{1}\left(d t d x, L_{v}^{1}\right)} .
$$

By Lemma 3.1(i) and Lemma 3.2(ii), the last two terms converge to 0 in $L_{\text {loc }}^{1}\left(d t d x, L_{v}^{1}\right)$.

$$
\varepsilon M \chi_{\varepsilon}^{\sharp} g_{\varepsilon}-M\left(1-\chi_{\varepsilon}\right)^{b} g_{\varepsilon}=\mathrm{O}(\varepsilon)_{L_{\mathrm{loc}}^{1}\left(d t d x, L_{v}^{1}\right)} .
$$

Define the function $\mathcal{M}$ by (4.2) and denote by $K=\left[\frac{1}{2}, \frac{3}{2}\right] \times\left[-\frac{1}{2}, \frac{1}{2}\right]^{d} \times\left[\frac{1}{2}, \frac{3}{2}\right]$. By Taylor's formula,

$$
\begin{gathered}
\chi_{\varepsilon}\left|M_{f_{\varepsilon}}-M-\left(\left(R_{\varepsilon}-1\right)+U_{\varepsilon} \cdot v+\left(T_{\varepsilon}-1\right) \frac{|v|^{2}-d}{2}\right) M\right| \\
\leqslant \chi_{\varepsilon}\left[\left(R_{\varepsilon}-1\right)^{2}+U_{\varepsilon}^{2}+\left(T_{\varepsilon}-1\right)^{2}\right] \sup _{K}\left|D_{(R, U, T)}^{2} \mathcal{M}\right| .
\end{gathered}
$$

Direct computations like in Section 4.2 show that for all $\alpha<\frac{2}{3}$, there exists $C_{\alpha}<+\infty$ such that, for all $v \in \mathbb{R}^{d}$,

$$
\sup _{K}\left|D_{(R, U, T)}^{2} \mathcal{M}\right| \leqslant C_{\alpha} M^{\alpha} .
$$

Then, by the above estimate and Lemma 3.2(ii), the first term in the right-side of (7.2) converges to 0 in $L_{\text {loc }}^{1}\left(d t, L_{x, v}^{1}\right)$

$$
\frac{\chi_{\varepsilon}}{\varepsilon}\left[M\left(\left(R_{\varepsilon}-1\right)+U_{\varepsilon} \cdot v+\left(T_{\varepsilon}-1\right) \frac{|v|^{2}-d}{2}\right)+M-M_{f_{\varepsilon}}\right]=\mathrm{O}(\varepsilon)_{L_{\mathrm{loc}}^{1}\left(d t, L_{x, v}^{1}\right)} .
$$

Finally, we get

$$
\frac{\chi_{\varepsilon}}{\varepsilon}\left(\left(R_{\varepsilon}-1\right)+U_{\varepsilon} \cdot v+\left(T_{\varepsilon}-1\right) \frac{|v|^{2}-d}{2}\right)-{ }^{b} g_{\varepsilon}=\mathrm{O}(\varepsilon) \quad \text { in } L_{\mathrm{loc}}^{1}\left(d t d x, L^{1}(M d v)\right)
$$

From Lemma 3.2(ii) we deduce that there exist $\rho, u, \theta \in L_{t}^{\infty}\left(L_{x}^{2}\right)$ such that, up to extraction of a subsequence,

$$
\chi_{\varepsilon} \varepsilon^{-1}\left(R_{\varepsilon}-1\right) \rightarrow \rho, \quad \chi_{\varepsilon} \varepsilon^{-1} U_{\varepsilon} \rightarrow u, \quad \chi_{\varepsilon} \varepsilon^{-1}\left(T_{\varepsilon}-1\right) \rightarrow \theta \quad \text { in } w-L_{t}^{\infty}\left(L_{x}^{2}\right) .
$$

Combining (7.3) and (7.4) gives the expected identity. 
In all the sequel, we will consider a sequence $\left(\varepsilon_{n}\right)$ such that $\varepsilon_{n} \rightarrow 0$ and

$$
\begin{aligned}
& { }^{b} g_{\varepsilon_{n}} \rightarrow g \text { in } w-L^{\infty}\left(d t, L^{2}(d x M d v)\right), \\
& R_{\varepsilon_{n}} \rightarrow 1 \text { almost everywhere on } \mathbb{R}^{+} \times \mathbb{R}^{d} \text { as } n \rightarrow \infty .
\end{aligned}
$$

LEMMA 7.2. - Let $\left(g_{\varepsilon}^{0}\right)$ be a family of measurable functions on $\mathbb{R}^{d} \times \mathbb{R}^{d}$ satisfying (1.13) and (1.14). For each $\varepsilon>0$, consider a solution $f_{\varepsilon}=M\left(1+\varepsilon g_{\varepsilon}\right)$ of (1.2). Consider a sequence $\left(\varepsilon_{n}\right)$ such that $\varepsilon_{n} \rightarrow 0$, satisfying (7.5). Then,

$$
\varepsilon^{-2}\left(M_{f_{\varepsilon}}-f_{\varepsilon}\right) \rightarrow v \cdot \nabla_{x} M g \quad \text { in } w-L_{\mathrm{loc}}^{1}\left(d t d x, L_{v}^{1}\right)
$$

and consequently, for all $t^{*}>0$,

$$
\left\|v \cdot \nabla_{x} g\right\|_{L^{2}\left(\left[0, t^{*}\right], L^{2}(d x M d v)\right)} \leqslant \liminf _{\varepsilon \rightarrow 0} \frac{1}{\varepsilon^{2}} H\left(M\left(1+\varepsilon g_{\varepsilon}^{0}\right) / M\right) \leqslant C_{0} .
$$

Proof. - By Lemma 4.1(ii) with $\eta_{\varepsilon}=1$, the sequence $\left(\varepsilon^{-2}\left(M_{f_{\varepsilon}}-f_{\varepsilon}\right)\right)$ is bounded in $L_{\text {loc }}^{1}\left(d t d x, L_{v}^{1}\right)$. Fix $\delta>0$ and let $\Omega=\left[0, t^{*}\right] \times \mathbb{R}^{d} \times \mathbb{R}^{d}$ be any bounded set of measure $|\Omega| \leqslant \delta^{2}$. By Lemma 4.1(ii) with $\eta_{\varepsilon}=\delta$, for all $\varepsilon \leqslant \delta$

$$
\iint_{0}^{t^{*}} \iint \frac{1}{\varepsilon^{2}}\left|M_{f_{\varepsilon}}-f_{\varepsilon}\right| \mathbb{1}_{\Omega} d x d v d t \leqslant C \delta\left\|\mathbb{1}_{\Omega}\right\|_{L_{t, x, v}^{\infty}}+\frac{C}{\delta}\left\|M \mathbb{1}_{\Omega}\right\|_{L_{t, x, v}^{1}} \leqslant \widetilde{C} \delta
$$

for some $C, \widetilde{C}>0$ which do not depend on $\delta$. The finite number of $\varepsilon_{n}$ with $\varepsilon_{n} \geqslant \delta$ can be accomodated by picking the measure of $\Omega$ sufficiently small. Then, the sequence $\left(\varepsilon^{-2}\left(M_{f_{\varepsilon}}-f_{\varepsilon}\right)\right)$ is locally equiintegrable in $L_{\text {loc }}^{1}\left(d t d x, L_{v}^{1}\right)$, and thus by Dunford-Pettis theorem relatively compact in $w-L_{\text {loc }}^{1}\left(d t d x, L_{v}^{1}\right)$.

Denote by $X$ any of its limit point. Taking limits in the kinetic equation

$$
\varepsilon \partial_{t}\left(M g_{\varepsilon}\right)+v \cdot \nabla_{x}\left(M g_{\varepsilon}\right)=\varepsilon^{-2}\left(M_{f_{\varepsilon}}-f_{\varepsilon}\right)
$$

provides then

$$
v \cdot \nabla_{x} M g=X .
$$

The uniqueness of the limit point, coupled with the compactness of the sequence, implies the convergence of the whole sequence $\left(\varepsilon^{-2}\left(M_{f_{\varepsilon}}-f_{\varepsilon}\right)\right)_{\varepsilon_{n}}$.

Taking limits in the dissipation bound provides then the second assertion in Lemma 7.2. Indeed, the convexity of $r$ gives the inequality

$$
\frac{f_{\varepsilon}}{\varepsilon^{4}} r\left(\varepsilon^{2} v \cdot \nabla_{x} g\right)+\frac{f_{\varepsilon}}{\varepsilon^{2}} r^{\prime}\left(\varepsilon^{2} v \cdot \nabla_{x} g\right)\left(\frac{f_{\varepsilon}-M_{f_{\varepsilon}}}{\varepsilon^{2} f_{\varepsilon}}-v \cdot \nabla_{x} g\right) \leqslant \frac{f_{\varepsilon}}{\varepsilon^{4}} r\left(\varepsilon^{2} \frac{f_{\varepsilon}-M_{f_{\varepsilon}}}{\varepsilon^{2} f_{\varepsilon}}\right) .
$$

Fix $\lambda>0$, multiply this inequality by the indicator function $\mathbb{1}_{\left|v \cdot \nabla_{x} g\right| \leqslant \lambda}$, average this over $\mathbb{R}^{d} \times \mathbb{R}^{d}$ and then consider its limit as $\varepsilon \rightarrow 0$. Use the strong $L^{\infty}$ limits

$$
\begin{aligned}
& \frac{1}{\varepsilon^{4}} r\left(\varepsilon^{2} v \cdot \nabla_{x} g\right) \mathbb{1}_{\left|v \cdot \nabla_{x} g\right| \leqslant \lambda} \rightarrow \frac{1}{2}\left(v \cdot \nabla_{x} g\right)^{2} \mathbb{1}_{\left|v \cdot \nabla_{x} g\right| \leqslant \lambda}, \\
& \frac{1}{\varepsilon^{2}} r^{\prime}\left(\varepsilon^{2} v \cdot \nabla_{x} g\right) \mathbb{1}_{\left|v \cdot \nabla_{x} g\right| \leqslant \lambda} \rightarrow v \cdot \nabla_{x} g \mathbb{1}_{\left|v \cdot \nabla_{x} g\right| \leqslant \lambda}
\end{aligned}
$$


and the weak $L^{1}$ limits

$$
f_{\varepsilon} \rightarrow M, \quad \frac{1}{\varepsilon^{2}}\left(f_{\varepsilon}-M_{f_{\varepsilon}}\right)-M v \cdot \nabla_{x} g \rightarrow 0
$$

to show that

$$
\iint M\left(v \cdot \nabla_{x} g\right)^{2} \mathbb{1}_{\left|v \cdot \nabla_{x} g\right| \leqslant \lambda} d x d v \leqslant \liminf _{\varepsilon \rightarrow 0} \frac{1}{\varepsilon^{4}} \iint f_{\varepsilon} r\left(\varepsilon^{2} \frac{f_{\varepsilon}-M_{f_{\varepsilon}}}{\varepsilon^{2} f_{\varepsilon}}\right) d x d v \leqslant C_{0} .
$$

Taking $\lambda \rightarrow+\infty$ completes the proof.

Lemmas 7.1 and 7.2 show together that the limiting form of the entropy inequality is essentially the Leray energy inequality for the limiting thermodynamic fields. In particular,

$$
v \cdot \nabla_{x} g=v \cdot \nabla_{x}\left(\rho+u \cdot v+\theta \frac{|v|^{2}-d}{2}\right)
$$

belongs to $L_{\text {loc }}^{2}\left(d t, L^{2}(d x M d v)\right)$, from which we deduce that $\nabla_{x} \rho,\left(\nabla_{x} u+\left(\nabla_{x} u\right)^{T}\right)$ and $\nabla_{x} \theta$ belong to $L_{\text {loc }}^{2}\left(d t, L_{x}^{2}\right)$.

\subsection{Incompressiblity and Boussinesq relations}

Taking limits in the local conservations of mass and momentum leads to the following constraints.

LEMMA 7.3. - Let $\left(g_{\varepsilon}^{0}\right)$ be a family of measurable functions on $\mathbb{R}^{d} \times \mathbb{R}^{d}$ satisfying (1.13) and (1.14). For each $\varepsilon>0$, consider a solution $f_{\varepsilon}=M\left(1+\varepsilon g_{\varepsilon}\right)$ of (1.2). Consider a sequence $\left(\varepsilon_{n}\right)$ such that $\varepsilon_{n} \rightarrow 0$, satisfying (7.5). Then g satisfies (7.1) as well as

$$
\nabla_{x} \cdot u=0, \quad \nabla_{x}(\rho+\theta)=0 .
$$

Proof. - By Theorem 2.3,

$$
\begin{aligned}
& \varepsilon \partial_{t}\left\langle{ }^{b} g_{\varepsilon}\right\rangle+\nabla_{x} \cdot\left\langle{ }^{b} g_{\varepsilon} v\right\rangle \rightarrow 0, \\
& \varepsilon \partial_{t}\left\langle{ }^{b} g_{\varepsilon} v\right\rangle+\nabla_{x} \cdot\left\langle{ }^{b} g_{\varepsilon} v \otimes v\right\rangle \rightarrow 0,
\end{aligned}
$$

in the sense of distributions. Then

$$
\nabla_{x} \cdot\langle g v\rangle=0, \quad \nabla_{x} \cdot\langle g v \otimes v\rangle=0
$$

which, coupled with (7.1), gives the expected result.

\subsection{Evolution equations}

It remains to prove that the limiting velocity $u$ satisfies the Navier-Stokes equation, while the limiting temperature $\theta$ is governed by the Fourier equation. By Theorem 2.3, the conservation defects for ${ }^{b} g_{\varepsilon}$ defined by (2.2) converge to 0 . Then,

$$
\begin{aligned}
& \partial_{t} P\left\langle{ }^{b} g_{\varepsilon} v\right\rangle+\frac{1}{\varepsilon} P \nabla_{x} \cdot\left\langle{ }^{b} g_{\varepsilon}\left(v \otimes v-\frac{|v|^{2}}{d} \mathrm{Id}\right)\right\rangle \rightarrow 0, \\
& \partial_{t}\left\langle{ }^{b} g_{\varepsilon}\left(\frac{|v|^{2}}{d+2}-1\right)\right\rangle+\frac{1}{\varepsilon} \nabla_{x} \cdot\left\langle{ }^{b} g_{\varepsilon}\left(\frac{|v|^{2}}{d+2}-1\right) v\right\rangle \rightarrow 0,
\end{aligned}
$$


where $P$ denotes the projection on the space of divergence-free vector fields. From the incompressibility and Boussinesq relations we deduce that $\partial_{t} P\left\langle{ }^{b} g_{\varepsilon} v\right\rangle$ and $\partial_{t}\left\langle\left\langle^{b} g_{\varepsilon}\left(\frac{|v|^{2}}{d+2}-1\right)\right\rangle\right.$ converge respectively to $\partial_{t} u$ and $\partial_{t} \theta$ in the sense of distributions. The difficulty is then to take limits in the flux terms. Denote by $\zeta(v)$ either the momentum flux $\left(v \otimes v-\frac{|v|^{2}}{d} \mathrm{Id}\right)$ or the heat flux $\left(\frac{|v|^{2}}{d+2}-1\right) v$. The following decomposition holds

$$
\begin{aligned}
\frac{1}{\varepsilon}\left\langle{ }^{b} g_{\varepsilon} \zeta(v)\right\rangle= & \frac{1-\chi_{\varepsilon}}{\varepsilon}\left\langle{ }^{b} g_{\varepsilon} \zeta(v)\right\rangle+\frac{\chi_{\varepsilon}}{\varepsilon^{2}} \int\left(f_{\varepsilon}-M_{f_{\varepsilon}}\right) \gamma\left(\frac{f_{\varepsilon}}{M}\right) \zeta(v) d v \\
& +\frac{\chi_{\varepsilon}}{\varepsilon^{2}} \int\left(M_{f_{\varepsilon}}-M\right) \gamma\left(\frac{f_{\varepsilon}}{M}\right) \zeta(v) d v .
\end{aligned}
$$

This paragraph is devoted to the study of each of these three terms, called in the sequel remainder, diffusion term and convection term.

LEMMA 7.4. - Let $\left(g_{\varepsilon}^{0}\right)$ be a family of measurable functions on $\mathbb{R}^{d} \times \mathbb{R}^{d}$ satisfying (1.13) and (1.14). For each $\varepsilon>0$, consider a solution $f_{\varepsilon}=M\left(1+\varepsilon g_{\varepsilon}\right)$ of (1.2), and define ${ }^{b} g_{\varepsilon}$ and ${ }^{\sharp} g_{\varepsilon}$ by (2.2) where $\gamma$ denotes any bump function satisfying (2.3). Consider a sequence $\left(\varepsilon_{n}\right)$ such that $\varepsilon_{n} \rightarrow 0$, satisfying (7.5). Denote by $\chi_{\varepsilon}$ the indicator function of $\left\{(t, x)|| R_{\varepsilon}-1\left|\leqslant \frac{1}{2},\right| U_{\varepsilon} \mid \leqslant \frac{1}{2}\right.$, $\left.\left|T_{\varepsilon}-1\right| \leqslant \frac{1}{2}\right\}$. Then,

$$
\frac{1-\chi_{\varepsilon}}{\varepsilon}\left\langle{ }^{b} g_{\varepsilon} \zeta(v)\right\rangle \rightarrow 0 \quad \text { in } w-L_{\mathrm{loc}}^{1}(d t d x)
$$

Proof. - We have

$$
\begin{aligned}
\left|\frac{1-\chi_{\varepsilon}}{\varepsilon}\left\langle{ }^{b} g_{\varepsilon} \zeta(v)\right\rangle\right| \leqslant & \frac{1-\chi_{\varepsilon}}{\varepsilon}\left\langle\left|g_{\varepsilon} \zeta(v)\right| \mathbb{1}_{|v|^{2} \leqslant \log |\log \varepsilon|}\right\rangle\|\gamma\|_{\infty} \\
& +\frac{1-\chi_{\varepsilon}}{\varepsilon}\left\langle\left|{ }^{b} g_{\varepsilon} \zeta(v)\right| \mathbb{1}_{|v|^{2}>\log |\log \varepsilon|}\right\rangle \\
\leqslant & C(\log |\log \varepsilon|)^{3 / 2} \int \frac{1-\chi_{\varepsilon}}{\varepsilon^{2}}\left|f_{\varepsilon}-M\right| d v \\
& +\frac{1-\chi_{\varepsilon}}{\varepsilon}\left\langle\zeta^{2}(v) \mathbb{1}_{|v|^{2}>\log |\log \varepsilon|}\right\rangle^{1 / 2}\left\langle{ }^{b} g_{\varepsilon}^{2}\right\rangle^{1 / 2}
\end{aligned}
$$

By Proposition 5.1(i), the first term in the right-side converges to 0 in $L_{\text {loc }}^{1}\left(d t, L_{x}^{1}\right)$. By Lemma 3.1(i) and Lemma 3.2(ii), the second term also converges to 0 in $L_{\text {loc }}^{1}\left(d t, L_{x}^{1}\right)$.

LEMMA 7.5. - Let $\left(g_{\varepsilon}^{0}\right)$ be a family of measurable functions on $\mathbb{R}^{d} \times \mathbb{R}^{d}$ satisfying (1.13) and (1.14). For each $\varepsilon>0$, consider a solution $f_{\varepsilon}=M\left(1+\varepsilon g_{\varepsilon}\right)$ of (1.2). Denote by $\gamma$ any bump function satisfying (2.3). Consider a sequence $\left(\varepsilon_{n}\right)$ such that $\varepsilon_{n} \rightarrow 0$, satisfying (7.5). Denote by $\chi_{\varepsilon}$ the indicator function of $\left\{(t, x)|| R_{\varepsilon}-1\left|\leqslant \frac{1}{2},\right| U_{\varepsilon}\left|\leqslant \frac{1}{2},\right| T_{\varepsilon}-1 \mid \leqslant \frac{1}{2}\right\}$. Then, up to extraction of subsequence,

$$
\frac{\chi_{\varepsilon}}{\varepsilon^{2}} \int\left(f_{\varepsilon}-M_{f_{\varepsilon}}\right) \gamma\left(\frac{f_{\varepsilon}}{M}\right) \zeta(v) d v \rightarrow-\nabla_{x} . \int M g v \zeta(v) d v \quad \text { in } w-L_{\mathrm{loc}}^{1}(d t d x)
$$

Proof. - Convergence (7.11) is a consequence of the two following assertions

$$
\begin{aligned}
\forall K>0, \quad \frac{\chi_{\varepsilon}}{\varepsilon^{2}} \int\left(f_{\varepsilon}-M_{f_{\varepsilon}}\right) \gamma\left(\frac{f_{\varepsilon}}{M}\right) \zeta(v) \mathbb{1}_{|v|^{2} \leqslant K} d v \\
\quad \rightarrow-\nabla_{x} . \int M g v \zeta(v) \mathbb{1}_{|v|^{2} \leqslant K} d v \quad \text { in } w-L_{\text {loc }}^{1}(d t d x) \text { as } \varepsilon \rightarrow 0,
\end{aligned}
$$




$$
\int M g v \zeta(v) \mathbb{1}_{|v|^{2} \geqslant K} d v \rightarrow 0 \quad \text { in } s-L_{\mathrm{loc}}^{1}(d t d x) \text { as } K \rightarrow \infty,
$$

(7.13) $\frac{\chi_{\varepsilon}}{\varepsilon^{2}} \int\left(f_{\varepsilon}-M_{f_{\varepsilon}}\right) \gamma\left(\frac{f_{\varepsilon}}{M}\right) \zeta(v) \mathbb{1}_{|v|^{2} \geqslant K} d v \rightarrow 0 \quad$ in $s-L_{\mathrm{loc}}^{1}(d t d x)$ as $K \rightarrow \infty, \varepsilon \rightarrow 0$.

- Lemmas 3.1 and 3.2 show that up to extraction of subsequence, $\chi_{\varepsilon} \gamma\left(\frac{f_{\varepsilon}}{M}\right)$ (which is bounded in $\left.L_{t, x, v}^{\infty}\right)$ converges a.e. to 1 . By Lemma 7.2, $\varepsilon^{-2}\left(f_{\varepsilon}-M_{f_{\varepsilon}}\right)$ converges to $-v \cdot \nabla_{x} M g$ in $w-L_{\text {loc }}^{1}\left(d t d x, L_{v}^{1}\right)$. Then the product limit theorem stated in Appendix $\mathrm{C}$ implies that convergence (7.12) holds in $w-L_{\text {loc }}^{1}(d t d x)$.

- The first assertion in (7.13) comes from the $L_{t}^{\infty}\left(L_{x, v}^{2}\right)$ bound on $M^{1 / 2} g$ and from trivial decay estimates on $M$. In order to establish the second assertion, we decompose the integral according to the velocity tails

$$
\begin{aligned}
& \iint_{0}^{t^{*}} \int_{Q} \frac{\chi_{\varepsilon}}{\varepsilon^{2}}\left|f_{\varepsilon}-M_{f_{\varepsilon}}\right| \gamma\left(\frac{f_{\varepsilon}}{M}\right)|\zeta(v)| \mathbb{1}_{|v|^{2} \geqslant K} d v d x d t \\
& \leqslant C \iiint_{Q} \frac{1}{\varepsilon^{2}}\left(f_{\varepsilon} \gamma\left(\frac{f_{\varepsilon}}{M}\right)+M_{f_{\varepsilon}} \chi_{\varepsilon}\right)|\zeta(v)| \mathbb{1}_{|v|^{2} \geqslant 8|\log \varepsilon|} d v d x d t \\
& +C \iint_{0} \int_{Q} \frac{\chi_{\varepsilon}}{\varepsilon^{2}}\left|f_{\varepsilon}-M_{f_{\varepsilon}}\right||\zeta(v)| \mathbb{1}_{K \leqslant|v|^{2} \leqslant 8|\log \varepsilon|} d v d x d t .
\end{aligned}
$$

For $\alpha<\frac{2}{3}$, there exist $C_{\alpha}, \widetilde{C}_{\alpha}<+\infty$ such that

$$
\iint_{0}^{t^{*}} \int_{Q} \frac{1}{\varepsilon^{2}}\left(f_{\varepsilon} \gamma\left(\frac{f_{\varepsilon}}{M}\right)+M_{f_{\varepsilon}} \chi_{\varepsilon}\right)|\zeta(v)| \mathbb{1}_{|v|^{2} \geqslant 8|\log \varepsilon|} d v d x d t
$$

$$
\leqslant \int_{0}^{t^{*}} \int_{Q} \frac{1}{\varepsilon^{2}}\left(\frac{3}{2} M+C_{\alpha} M^{\alpha}\right)|\zeta(v)| \mathbb{1}_{|v|^{2} \geqslant 8|\log \varepsilon|} d v d x d t \leqslant \widetilde{C}_{\alpha} \varepsilon^{4 \alpha-2}|\log \varepsilon|^{(1+d) / 2} .
$$

Decompose $\frac{1}{\varepsilon^{2}}\left(f_{\varepsilon}-M_{f_{\varepsilon}}\right)=M_{f_{\varepsilon}}\left({ }^{b} q_{\varepsilon}+\varepsilon^{2 \sharp} q_{\varepsilon}\right)$ as in (2.5) where ${ }^{b} q_{\varepsilon}$ and ${ }^{\sharp} q_{\varepsilon}$ satisfy (5.24). Then, by Cauchy-Schwarz inequality,

$$
\begin{aligned}
& \iint_{0}^{t^{*}} \int_{Q} \frac{\chi_{\varepsilon}}{\varepsilon^{2}}\left|f_{\varepsilon}-M_{f_{\varepsilon}}\right||\zeta(v)| \mathbb{1}_{K \leqslant|v|^{2} \leqslant 8|\log \varepsilon|} d v d x d t \\
& \leqslant\left.\int_{0} \iint_{Q} \chi_{\varepsilon} M_{f_{\varepsilon}}\right|^{b} q_{\varepsilon}|| \zeta(v) \mid \mathbb{1}_{K \leqslant|v|^{2}} d v d x d t \\
& \quad+\varepsilon^{2} \iint_{0}^{t_{Q}^{*}} M_{f_{\varepsilon}}\left|{ }^{\sharp} q_{\varepsilon}\right||\zeta(v)| \mathbb{1}_{|v|^{2} \leqslant 8|\log \varepsilon|} d v d x d t \\
& \leqslant\left(\left.\left.\int_{0}^{t^{*}} \iint_{Q} M_{f_{\varepsilon}}\right|^{b} q_{\varepsilon}\right|^{2} d v d x d t\right)^{1 / 2}\left(\iint_{0}^{t^{*}} \int_{Q} \chi_{\varepsilon} M_{f_{\varepsilon}}|\zeta(v)|^{2} \mathbb{1}_{K \leqslant|v|^{2}} d v d x d t\right)^{1 / 2}
\end{aligned}
$$




$$
\begin{gathered}
+\varepsilon^{2}\left\|M_{f_{\varepsilon}}^{\sharp} q_{\varepsilon}\right\|_{L^{1}\left(\left[0, t^{*}\right], L_{x, v}^{1}\right)}\left\|\zeta(v) \mathbb{1}_{|v|^{2} \leqslant 8|\log \varepsilon|}\right\|_{L_{v}^{\infty}} \\
\leqslant C_{\alpha}\left(\int M^{\alpha}\left(1+|v|^{6}\right) \mathbb{1}_{|v|^{2} \geqslant K} d v\right)^{1 / 2}+C \varepsilon^{2}(8|\log \varepsilon|)^{3 / 2}
\end{gathered}
$$

for some $C, C_{\alpha}>0$. Combining (7.14)-(7.16) and choosing $\alpha>\frac{1}{2}$ lead to (7.13).

LEMMA 7.6. - Let $\left(g_{\varepsilon}^{0}\right)$ be a family of measurable functions on $\mathbb{R}^{d} \times \mathbb{R}^{d}$ satisfying (1.13) and (1.14). For each $\varepsilon>0$, consider a solution $f_{\varepsilon}=M\left(1+\varepsilon g_{\varepsilon}\right)$ of (1.2). Denote by $\gamma$ any bump function satisfying (2.3). Consider a sequence $\left(\varepsilon_{n}\right)$ such that $\varepsilon_{n} \rightarrow 0$, satisfying (7.5). Denote by $\chi_{\varepsilon}$ the indicator function of $\left\{(t, x)|| R_{\varepsilon}-1\left|\leqslant \frac{1}{2},\right| U_{\varepsilon}\left|\leqslant \frac{1}{2},\right| T_{\varepsilon}-1 \mid \leqslant \frac{1}{2}\right\}$. Denote by $u$ and $\theta$ the limiting velocity and temperature defined by (7.1). Then, up to extraction of subsequence,

$$
P \nabla_{x} . \int \frac{\chi_{\varepsilon}}{\varepsilon^{2}}\left(M_{f_{\varepsilon}}-M\right) \gamma\left(\frac{f_{\varepsilon}}{M}\right)\left(v \otimes v-\frac{|v|^{2}}{d} \mathrm{Id}\right) d v \rightarrow P \nabla_{x} .(u \otimes u) \quad \text { in } \mathcal{D}_{t, x}^{\prime}
$$

where P denotes the Leray projection onto the divergence-free vector fields, and

$$
\nabla_{x} . \int \frac{\chi_{\varepsilon}}{\varepsilon^{2}}\left(M_{f_{\varepsilon}}-M\right) \gamma\left(\frac{f_{\varepsilon}}{M}\right)\left(\frac{|v|^{2}}{d+2}-1\right) v d v \rightarrow \nabla_{x} .(\theta u) \quad \text { in } \mathcal{D}_{t, x}^{\prime} .
$$

Proof. - The first step of the proof is to establish the convergence of the remainders

$$
\begin{aligned}
& \int \frac{\chi_{\varepsilon}}{\varepsilon^{2}}\left(M_{f_{\varepsilon}}-M\right) \gamma\left(\frac{f_{\varepsilon}}{M}\right)\left(v \otimes v-\frac{|v|^{2}}{d} \operatorname{Id}\right) d v-\left({ }^{b} u_{\varepsilon} \otimes{ }^{b} u_{\varepsilon}-\frac{\left|{ }^{b} u_{\varepsilon}\right|^{2}}{d} \operatorname{Id}\right) \rightarrow 0, \\
& \int \frac{\chi_{\varepsilon}}{\varepsilon^{2}}\left(M_{f_{\varepsilon}}-M\right) \gamma\left(\frac{f_{\varepsilon}}{M}\right)\left(\frac{|v|^{2}}{d+2}-1\right) v d v-\left({ }^{b} \theta_{\varepsilon}{ }^{b} u_{\varepsilon}\right) \rightarrow 0,
\end{aligned}
$$

where ${ }^{b} u_{\varepsilon}=\left\langle{ }^{b} g_{\varepsilon} v\right\rangle$ and ${ }^{b} \theta_{\varepsilon}=\frac{1}{d}\left\langle{ }^{b} g_{\varepsilon}\left(|v|^{2}-d\right)\right\rangle$. Then we will prove that

$$
P \nabla_{x} \cdot\left({ }^{b} u_{\varepsilon} \otimes{ }^{b} u_{\varepsilon}\right) \rightarrow P \nabla_{x} \cdot(u \otimes u), \quad \nabla_{x} \cdot\left({ }^{b} \theta_{\varepsilon}^{b} u_{\varepsilon}\right) \rightarrow \nabla_{x} \cdot(\theta u) .
$$

The difficulty in this second part will be the lack of compactness of $\left({ }^{b} u_{\varepsilon}\right)$ and $\left({ }^{b} \theta_{\varepsilon}\right)$ with respect to time, due to the presence of high frequency oscillations (acoustic waves).

- Denote by $\zeta(v)$ either $\left(v \otimes v-\frac{|v|^{2}}{d} \mathrm{Id}\right)$ or $\left(\frac{|v|^{2}}{d+2}-1\right) v$. As $\int M \zeta(v) d v=0$,

$$
\begin{aligned}
\int \frac{\chi_{\varepsilon}}{\varepsilon^{2}}\left(M_{f_{\varepsilon}}-M\right) \gamma\left(\frac{f_{\varepsilon}}{M}\right) \zeta(v) d v \\
=\int \frac{\chi_{\varepsilon}}{\varepsilon^{2}}\left(M_{f_{\varepsilon}}-M\right)\left(\gamma\left(\frac{f_{\varepsilon}}{M}\right)-1\right) \zeta(v) d v+\int \frac{\chi_{\varepsilon}}{\varepsilon^{2}} M_{f_{\varepsilon}} \zeta(v) d v .
\end{aligned}
$$

By Lemma 3.1(iii), Lemma 4.2 and Proposition 5.3, the quantity

$$
\begin{gathered}
\iint_{0}^{t^{*}} \int_{Q} \frac{\chi_{\varepsilon}}{\varepsilon^{2}}\left|M_{f_{\varepsilon}}-M\right|\left|\gamma\left(\frac{f_{\varepsilon}}{M}\right)-1\right||\zeta(v)| d v d x d t \\
\quad \leqslant\left(\iiint_{0}^{t^{*}} \chi_{\varepsilon} \frac{\left|M_{f_{\varepsilon}}-M\right|^{2}}{\varepsilon^{2} M}|\zeta(v)|^{2} d v d x d t\right)^{1 / 2}
\end{gathered}
$$

$4^{\mathrm{e}}$ SÉRIE - TOME $36-2003-\mathrm{N}^{\circ} 2$ 


$$
\begin{aligned}
& \times\left(\iint_{0}^{t^{*}} \int_{Q} \frac{M}{\varepsilon^{2}}\left|\gamma\left(\frac{f_{\varepsilon}}{M}\right)-1\right|^{2} d v d x d t\right)^{1 / 2} \\
\leqslant & \left(\iint_{0}^{t^{*}} \int_{Q} \chi_{\varepsilon} \frac{\left|M_{f_{\varepsilon}}-M\right|^{2}}{\varepsilon^{2} M}|\zeta(v)|^{2} d v d x d t\right)^{1 / 2} \\
& \times\left(\left.\iiint_{0}^{t^{*}} 9\|1-\gamma\|_{\infty} M\right|^{\sharp} g_{\varepsilon} \mid d v d x d t\right)^{1 / 2}
\end{aligned}
$$

converges to 0 as $\varepsilon \rightarrow 0$. On the other hand, direct computations give

$$
\begin{aligned}
\int & \frac{\chi_{\varepsilon}}{\varepsilon^{2}} M_{f_{\varepsilon}}\left(v \otimes v-\frac{|v|^{2}}{d} \mathrm{Id}\right) d v \\
= & \frac{\chi_{\varepsilon}}{\varepsilon^{2}} R_{\varepsilon}\left(U_{\varepsilon} \otimes U_{\varepsilon}-\frac{\left|U_{\varepsilon}\right|^{2}}{d} \mathrm{Id}\right) \\
= & \frac{\chi_{\varepsilon}}{R_{\varepsilon}}\left(\left({ }^{b} u_{\varepsilon}+\varepsilon^{\sharp} u_{\varepsilon}\right) \otimes\left({ }^{b} u_{\varepsilon}+\varepsilon^{\sharp} u_{\varepsilon}\right)-\frac{\left|{ }^{b} u_{\varepsilon}+\varepsilon^{\sharp} u_{\varepsilon}\right|^{2}}{d} \mathrm{Id}\right) .
\end{aligned}
$$

Then,

$$
\begin{aligned}
& \left\|\int \frac{\chi_{\varepsilon}}{\varepsilon^{2}} M_{f_{\varepsilon}}\left(v \otimes v-\frac{|v|^{2}}{d} \mathrm{Id}\right) d v-\left({ }^{b} u_{\varepsilon} \otimes{ }^{b} u_{\varepsilon}-\frac{\left|{ }^{b} u_{\varepsilon}\right|^{2}}{d} \mathrm{Id}\right)\right\|_{L^{1}\left(\left[0, t^{*}\right] \times Q\right)} \\
& \leqslant C\left\|\left(\frac{\chi_{\varepsilon}}{R_{\varepsilon}}-1\right)^{b} u_{\varepsilon}^{2}\right\|_{L^{1}\left(\left[0, t^{*}\right] \times Q\right)}+C\left\|\chi_{\varepsilon}^{\sharp} u_{\varepsilon}\right\|_{L^{1}([0, T] \times Q)}\left\|\left.\varepsilon^{2} \chi_{\varepsilon}\right|^{\sharp} u_{\varepsilon}|+\varepsilon|^{b} u_{\varepsilon} \mid\right\|_{L^{\infty}} .
\end{aligned}
$$

By Proposition 5.4(ii) and Cauchy-Schwarz inequality

$$
\left({ }^{b} u_{\varepsilon}\right)^{2} \leqslant\left(\int M^{b} g_{\varepsilon}^{2} d v\right)\left(\int M|v|^{4} d v\right) \text { a.e. on } \mathbb{R}^{+} \times \mathbb{R}^{d},
$$

$\left({ }^{b} u_{\varepsilon}^{2}\right)$ is relatively compact in $w-L_{\text {loc }}^{1}(d t d x)$. As $\left(\frac{\chi_{\varepsilon}}{R_{\varepsilon}}-1\right)$ is bounded in $L_{t, x}^{\infty}$ and converges a.e. to 0 , the product limit theorem (Appendix C) shows that up to extraction of subsequence, the first term in the right-side of (7.23) converges to 0. By Proposition 5.3(iii), $\chi_{\varepsilon}^{\sharp} u_{\varepsilon}$ converges to 0 in $L_{\text {loc }}^{1}(d t d x)$. By definition of $\chi_{\varepsilon}$,

$$
\frac{K_{\varepsilon}}{R_{\varepsilon}} \leqslant 2 R_{\varepsilon}, \quad \chi_{\varepsilon}\left|\varepsilon^{b} u_{\varepsilon}+\varepsilon^{2 \sharp} u_{\varepsilon}\right| \leqslant \chi_{\varepsilon}\left|U_{\varepsilon}\right| \leqslant \frac{1}{2}
$$

while by Lemma 3.1(iii),

$$
\left|\varepsilon^{b} g_{\varepsilon}\right| \leqslant\left\|\varepsilon^{b} g_{\varepsilon}\right\|_{L_{t, x, v}^{\infty}} \int M|v| d v \leqslant C .
$$

Then the second term in the right-side of (7.23) converges to 0. Combining (7.21)-(7.23) provides the first convergence in (7.19). In the same way,

$$
\int \frac{\chi_{\varepsilon}}{\varepsilon^{2}} M_{f_{\varepsilon}}\left(\frac{|v|^{2}}{d+2}-1\right) v d v
$$




$$
\begin{aligned}
& =\frac{\chi_{\varepsilon}}{\varepsilon^{2}}\left(R_{\varepsilon}\left(T_{\varepsilon}-1\right) U_{\varepsilon}+\frac{1}{d+2} R_{\varepsilon}\left|U_{\varepsilon}\right|^{2} U_{\varepsilon}\right) \\
& =\frac{\chi_{\varepsilon}}{R_{\varepsilon}}\left({ }^{b} u_{\varepsilon}+\varepsilon^{\sharp} u_{\varepsilon}\right)\left(\left({ }^{b} \theta_{\varepsilon}+\varepsilon^{\sharp} \theta_{\varepsilon}\right)-\left.\frac{2 \varepsilon}{d(d+2) R_{\varepsilon}}\right|^{b} u_{\varepsilon}+\left.\varepsilon^{\sharp} u_{\varepsilon}\right|^{2}\right) .
\end{aligned}
$$

Then

$$
\begin{aligned}
& \left\|\int \frac{\chi_{\varepsilon}}{\varepsilon^{2}} M_{f_{\varepsilon}}\left(\frac{|v|^{2}}{d+2}-1\right) v d v-\left({ }^{b} \theta_{\varepsilon}^{b} u_{\varepsilon}\right)\right\|_{L^{1}\left(\left[0, t^{*}\right] \times Q\right)} \\
& \leqslant C\left\|\left(\frac{\chi_{\varepsilon}}{R_{\varepsilon}}-1\right)^{b} u_{\varepsilon}^{b} \theta_{\varepsilon}\right\|_{L^{1}\left(\left[0, t^{*}\right] \times Q\right)}+C\left\|\left(\varepsilon^{b} u_{\varepsilon}\right)^{b} u_{\varepsilon}^{2}\right\|_{L^{1}\left(\left[0, t^{*}\right] \times Q\right)} \\
& \quad+C\left(\left\|\chi_{\varepsilon}^{\sharp} u_{\varepsilon}\right\|_{L^{1}\left(\left[0, t^{*}\right] \times Q\right)}+\left\|\chi_{\varepsilon}^{\sharp} \theta_{\varepsilon}\right\|_{L^{1}\left(\left[0, t^{*}\right] \times Q\right)}\right) \\
& \quad \times\left(\left\|\left.\varepsilon^{2} \chi_{\varepsilon}\right|^{\sharp} u_{\varepsilon}|+\varepsilon|^{b} u_{\varepsilon}\left|\left\|_{L^{\infty}}^{2}+\right\| \varepsilon^{2} \chi_{\varepsilon}\right|^{\sharp} \theta_{\varepsilon}|+\varepsilon|^{b} \theta_{\varepsilon} \mid\right\|_{L^{\infty}}\right)
\end{aligned}
$$

converges to 0 by the same arguments as previously. Combining (7.21), (7.22) and (7.24) gives the second convergence in (7.19).

- It remains to establish the convergence of the quadratic terms $P \nabla_{x} \cdot\left({ }^{b} u_{\varepsilon} \otimes{ }^{b} u_{\varepsilon}\right)$ and $\nabla_{x} \cdot\left({ }^{b} u_{\varepsilon}^{b} \theta_{\varepsilon}\right)$. Combining (7.8)-(7.11) and (7.19) gives

$$
\begin{aligned}
& \partial_{t}\left(P^{b} u_{\varepsilon}\right)+P \nabla_{x} \cdot\left({ }^{b} u_{\varepsilon} \otimes^{b} u_{\varepsilon}\right) \text { uniformly bounded in } L_{\mathrm{loc}}^{1}\left(d t, W_{\mathrm{loc}}^{-1-s, 1}(d x)\right), \quad s>0, \\
\text { (7.25) } & \partial_{t}\left(\frac{d^{b} \theta_{\varepsilon}-2^{b} \rho_{\varepsilon}}{d+2}\right)+\nabla_{x} \cdot\left({ }^{b} u_{\varepsilon}^{b} \theta_{\varepsilon}\right) \text { uniformly bounded in } L_{\mathrm{loc}}^{1}\left(d t, W_{\mathrm{loc}}^{-1,1}(d x)\right)
\end{aligned}
$$

which implies that $\left(\partial_{t}\left(P^{b} u_{\varepsilon}\right)\right)$ and $\left(\partial_{t}\left(\frac{d^{b} \theta_{\varepsilon}-2^{\mathrm{b}} \rho_{\varepsilon}}{d+2}\right)\right)$ are bounded in $L_{\text {loc }}^{1}\left(d t, W_{\text {loc }}^{-1-s, 1}(d x)\right)$. From this bound and Corollary 5.5, we deduce by a standard interpolation argument that both sequences $\left(P^{\mathrm{b}} u_{\varepsilon}\right)$ and $\left(\frac{d^{\mathrm{b}} \theta_{\varepsilon}-2^{\mathrm{b}} \rho_{\varepsilon}}{d+2}\right)$ are relatively strongly compact in $L_{\text {loc }}^{2}(d t d x)$. By the incompressibility and Boussinesq relations,

$$
P^{b} u_{\varepsilon} \rightarrow u, \quad \frac{d^{b} \theta_{\varepsilon}-2^{b} \rho_{\varepsilon}}{d+2} \rightarrow \theta \quad \text { strongly in } L_{\mathrm{loc}}^{2}(d t d x) .
$$

Denote by $\nabla_{x} \psi_{\varepsilon}={ }^{b} u_{\varepsilon}-P^{b} u_{\varepsilon}$

(7.27) $\nabla_{x} \psi_{\varepsilon} \rightarrow 0$

$$
\frac{2}{d+2}\left({ }^{b} \rho_{\varepsilon}+{ }^{b} \theta_{\varepsilon}\right)={ }^{b} \theta_{\varepsilon}-\frac{d^{b} \theta_{\varepsilon}-2^{b} \rho_{\varepsilon}}{d+2} \rightarrow 0 \quad \text { in } w-L_{\mathrm{loc}}^{2}(d t d x) .
$$

Then, by (7.26), (7.27),

$$
\begin{aligned}
& P \nabla_{x} \cdot\left({ }^{b} u_{\varepsilon} \otimes{ }^{b} u_{\varepsilon}\right)-P \nabla_{x} \cdot\left(\nabla_{x} \psi_{\varepsilon} \otimes \nabla_{x} \psi_{\varepsilon}\right)-P \nabla_{x} \cdot(u \otimes u) \rightarrow 0, \\
& \nabla_{x} \cdot\left({ }^{b} u_{\varepsilon}^{b} \theta_{\varepsilon}\right)-\frac{2}{d+2} \nabla_{x} \cdot\left(\left({ }^{b} \rho_{\varepsilon}+{ }^{b} \theta_{\varepsilon}\right) \nabla_{x} \psi_{\varepsilon}\right)-\nabla_{x} \cdot(u \theta) \rightarrow 0,
\end{aligned}
$$

in the sense of distributions.

It remains to prove that $P \nabla_{x} \cdot\left(\nabla_{x} \psi_{\varepsilon} \otimes \nabla_{x} \psi_{\varepsilon}\right)$ and $\frac{2}{d+2} \nabla_{x} \cdot\left(\left({ }^{b} \rho_{\varepsilon}+{ }^{b} \theta_{\varepsilon}\right) \nabla_{x} \psi_{\varepsilon}\right)$ converge to 0 . Indeed we use the trick introduced independently by Schochet and Grenier [25,16] to study the fast singular limits of hyperbolic PDEs, adapted to the case of acoustic waves by Lions and Masmoudi [19]. First we introduce the following regularization: let $\kappa \in C_{c}^{\infty}\left(\mathbb{R}^{d}\right)$ such that $\kappa(x)=0$ if $|x| \geqslant 1$ and $\int \kappa d x=1$, we define $\kappa_{\delta}(x)=\delta^{-d} \kappa(x / \delta)$ and

$$
\psi_{\varepsilon}^{\delta}=\kappa_{\delta} * \psi_{\varepsilon}, \quad \pi_{\varepsilon}^{\delta}=\kappa_{\delta} *\left({ }^{b} \rho_{\varepsilon}+{ }^{b} \theta_{\varepsilon}\right) .
$$


By Corollary 5.5, for all $t^{*}>0$ and all $Q$ bounded subset of $\mathbb{R}^{d}$, there exists a nonnegative function $\eta \in C\left(\mathbb{R}^{+}\right)$with $\eta(0)=0$ such that all $y \in B(0,1)$

$$
\begin{aligned}
& \left\|\nabla_{x} \psi_{\varepsilon}(t, x+\delta y)-\nabla_{x} \psi_{\varepsilon}(t, x)\right\|_{L^{2}\left(\left[0, t^{*}\right] \times Q\right)} \leqslant \eta(\delta), \\
& \left\|\left({ }^{b} \rho_{\varepsilon}+{ }^{b} \theta_{\varepsilon}\right)(t, x+\delta y)-\left({ }^{b} \rho_{\varepsilon}+{ }^{b} \theta_{\varepsilon}\right)(t, x)\right\|_{L^{2}\left(\left[0, t^{*}\right] \times Q\right)} \leqslant \eta(\delta),
\end{aligned}
$$

which imply that uniformly in $\varepsilon$,

$$
\left\|\nabla_{x} \psi_{\varepsilon}^{\delta}-\nabla_{x} \psi_{\varepsilon}\right\|_{L^{2}\left(\left[0, t^{*}\right] \times Q\right)} \leqslant C \sup _{[0, \delta]} \eta, \quad\left\|\pi_{\varepsilon}^{\delta}-\left({ }^{b} \rho_{\varepsilon}+{ }^{b} \theta_{\varepsilon}\right)\right\|_{L^{2}\left(\left[0, t^{*}\right] \times Q\right)} \leqslant C \sup _{[0, \delta]} \eta .
$$

Hence, in the sense of distributions,

$$
P \nabla_{x} \cdot\left(\nabla_{x} \psi_{\varepsilon} \otimes \nabla_{x} \psi_{\varepsilon}\right)-P \nabla_{x} \cdot\left(\nabla_{x} \psi_{\varepsilon}^{\delta} \otimes \nabla_{x} \psi_{\varepsilon}^{\delta}\right) \rightarrow 0
$$

(7.29) $\frac{2}{d+2} \nabla_{x} \cdot\left(\left({ }^{b} \rho_{\varepsilon}+{ }^{b} \theta_{\varepsilon}\right) \nabla_{x} \psi_{\varepsilon}\right)-\frac{2}{d+2} \nabla_{x} \cdot\left(\pi_{\varepsilon}^{\delta} \nabla_{x} \psi_{\varepsilon}^{\delta}\right) \rightarrow 0 \quad$ uniformly in $\varepsilon$ as $\delta \rightarrow 0$.

From the uniform bounds

$$
\left\|\nabla_{x} \psi_{\varepsilon}\right\|_{L_{t}^{\infty}\left(L_{x}^{2}\right)} \leqslant C, \quad\left\|{ }^{b} \rho_{\varepsilon}+{ }^{b} \theta_{\varepsilon}\right\|_{L_{t}^{\infty}\left(L_{x}^{2}\right)} \leqslant C
$$

we deduce that for all $\delta>0$ and $s>0$

$$
\nabla_{x} \psi_{\varepsilon}^{\delta}, \pi_{\varepsilon}^{\delta} \text { are bounded in } L_{t}^{\infty}\left(H_{\mathrm{loc}}^{s}(d x)\right) \text { uniformly in } \varepsilon \text {. }
$$

Theorem 2.3 coupled with (7.8)-(7.11) and (7.19) gives

$$
\begin{aligned}
& \varepsilon \partial_{t}^{b} u_{\varepsilon}+\nabla_{x}\left({ }^{b} \rho_{\varepsilon}+{ }^{b} \theta_{\varepsilon}\right) \rightarrow 0, \\
& \varepsilon \partial_{t}\left({ }^{b} \rho_{\varepsilon}+{ }^{b} \theta_{\varepsilon}\right)+\frac{d+2}{d} \nabla_{x} \cdot{ }^{b} u_{\varepsilon} \rightarrow 0 \quad \text { in } L_{\text {loc }}^{1}\left(d t, W_{\text {loc }}^{-1,1}(d x)\right)
\end{aligned}
$$

from which we deduce that for all $\delta>0$ and $s>0$,

$$
\begin{aligned}
& S_{\varepsilon}^{\delta}=\varepsilon \partial_{t} \nabla_{x} \psi_{\varepsilon}^{\delta}+\nabla_{x} \pi_{\varepsilon}^{\delta} \rightarrow 0, \\
& {S^{\prime}}_{\varepsilon}^{\delta}=\varepsilon \partial_{t} \pi_{\varepsilon}^{\delta}+\frac{d+2}{d} \Delta_{x} \psi_{\varepsilon}^{\delta} \rightarrow 0 \quad \text { in } L_{\mathrm{loc}}^{1}\left(d t, H_{\mathrm{loc}}^{s}(d x)\right) .
\end{aligned}
$$

Then, the following computations

$$
\begin{aligned}
P \nabla_{x} \cdot\left(\nabla_{x} \psi_{\varepsilon}^{\delta} \otimes \nabla_{x} \psi_{\varepsilon}^{\delta}\right) & =\frac{1}{2} P \nabla_{x}\left|\nabla_{x} \psi_{\varepsilon}^{\delta}\right|^{2}+P\left(\Delta_{x} \psi_{\varepsilon}^{\delta} \nabla_{x} \psi_{\varepsilon}^{\delta}\right) \\
& =\frac{d}{d+2} P\left(-\partial_{t}\left(\varepsilon \pi_{\varepsilon}^{\delta} \nabla_{x} \psi_{\varepsilon}^{\delta}\right)-\pi_{\varepsilon}^{\delta} \nabla_{x} \pi_{\varepsilon}^{\delta}+\pi_{\varepsilon}^{\delta} S_{\varepsilon}^{\delta}+{S^{\prime}}_{\varepsilon}^{\delta} \nabla_{x} \psi_{\varepsilon}^{\delta}\right) \\
& =\frac{d}{d+2} P\left(-\partial_{t}\left(\varepsilon \pi_{\varepsilon}^{\delta} \nabla_{x} \psi_{\varepsilon}^{\delta}\right)+\pi_{\varepsilon}^{\delta} S_{\varepsilon}^{\delta}+S_{\varepsilon}^{\prime \delta} \nabla_{x} \psi_{\varepsilon}^{\delta}\right), \\
\nabla_{x} \cdot\left(\pi_{\varepsilon}^{\delta} \nabla_{x} \psi_{\varepsilon}^{\delta}\right) & =\pi_{\varepsilon}^{\delta} \Delta_{x} \psi_{\varepsilon}^{\delta}+\nabla_{x} \psi_{\varepsilon}^{\delta} \cdot \nabla_{x} \pi_{\varepsilon}^{\delta} \\
& =\frac{d}{d+2} \pi_{\varepsilon}^{\delta}\left({S^{\prime}}_{\varepsilon}^{\delta}-\varepsilon \partial_{t} \pi_{\varepsilon}^{\delta}\right)+\nabla_{x} \psi_{\varepsilon}^{\delta} \cdot\left(S_{\varepsilon}^{\delta}-\varepsilon \partial_{t} \nabla_{x} \psi_{\varepsilon}^{\delta}\right) \\
& =\frac{d}{d+2} \pi_{\varepsilon}^{\delta}{S^{\prime}}_{\varepsilon}^{\delta}+\nabla_{x} \psi_{\varepsilon}^{\delta} \cdot S_{\varepsilon}^{\delta}-\frac{d \varepsilon}{2(d+2)} \partial_{t}\left|\pi_{\varepsilon}^{\delta}\right|^{2}-\frac{\varepsilon}{2} \partial_{t}\left|\nabla_{x} \psi_{\varepsilon}^{\delta}\right|^{2}
\end{aligned}
$$

coupled with (7.30) and (7.31), show that, for all $\delta>0$, both terms converge to 0 in the sense of distributions as $\varepsilon \rightarrow 0$. Combining (7.28), (7.29) and (7.32) gives (7.20), which coupled with (7.19) provides (7.17) and (7.18). 


\section{Appendix A. Young's inequality}

The functions $h:]-1,+\infty\left[\rightarrow \mathbb{R}_{+}\right.$and $\left.r:\right]-1,+\infty\left[\rightarrow \mathbb{R}_{+}\right.$are both strictly convex, and satisfy, for all $z>-1$,

$$
h(|z|) \leqslant h(z), \quad r(|z|) \leqslant r(z), \quad h(z) \leqslant r(z) .
$$

The Legendre transform of $h$ is defined for all $p \in \mathbb{R}$ by

$$
h^{*}(p)=\sup _{z>-1}(p z-h(z))=e^{p}-p-1
$$

that of $r$ is also defined for all $p \in \mathbb{R}$ by the implicit relation

$$
r^{*}(p)=\sup _{z>-1}(p z-r(z))=\frac{z^{2}}{1+z}, \quad \text { with } \log (1+z)+\frac{z}{1+z}=p .
$$

Further, the Legendre transform $h^{*}$ is super-quadratic, i.e.

$$
h^{*}(\lambda p) \leqslant \lambda^{2} h^{*}(p), \quad p \in \mathbb{R}, \lambda \in[0,1] .
$$

Finally Young's inequality states that, for all $p \in \mathbb{R}, z>-1$ and $\lambda \in[0,1]$,

$$
p|z| \leqslant \frac{1}{\lambda} h(z)+\lambda h^{*}(p) \leqslant \frac{1}{\lambda} r(z)+\lambda h^{*}(p) .
$$

\section{Appendix B. Velocity averaging}

Proposition B.1. - Let $\phi_{\varepsilon}$ be a family of $L_{\mathrm{loc}}^{2}\left(d t d x, L^{2}(M d v)\right)$ indexed by $\varepsilon \in[0,1]$ such that the families $\left|\phi_{\varepsilon}\right|^{2}$ and $\left(\varepsilon \partial_{t}+v \cdot \nabla_{x}\right) \phi_{\varepsilon}$ are both relatively compact in $w-$ $L_{\text {loc }}^{1}\left(d x d t, L^{2}(M d v)\right)$. Then, for each function $\psi \equiv \psi(v)$ in $L^{2}(M d v)$, each $T>0$ and each compact $Q \subset \mathbb{R}^{d}$, there exists a function $\eta$ in $C\left(\mathbb{R}_{+}\right)$such that

$$
\left\|\int \phi_{\varepsilon}(t, x+\delta y, v) \psi(v) M(v) d v-\int \phi_{\varepsilon}(t, x, v) \psi(v) M(v) d v\right\|_{L^{2}([0, T] \times Q)} \leqslant \eta(\delta)
$$

for each $\delta>0, y \in B(0,1)$, uniformly in $\varepsilon \in[0,1]$.

Proof. - Without loss of generality, we can assume that $\psi \in C_{c}\left(\mathbb{R}^{d}\right)$ and that all the $\phi_{\varepsilon}$ are supported in some compact $K \subset \mathbb{R}_{+}^{*} \times \mathbb{R}^{d} \times \mathbb{R}^{d}$; thus we henceforth consider $\psi_{\varepsilon}$ as defined on $\mathbb{R} \times \mathbb{R}^{d} \times \mathbb{R}^{d}$. Let $\lambda>0$; define

$$
\phi_{\varepsilon}+\left(\varepsilon \partial_{t}+v \cdot \nabla_{x}\right) \phi_{\varepsilon}=\Phi_{\varepsilon}
$$

and

$$
\begin{aligned}
& \phi_{\varepsilon}^{<}=\left(1+\varepsilon \partial_{t}+v \cdot \nabla_{x}\right)^{-1}\left(\Phi_{\varepsilon} \mathbb{1}_{\left|\Phi_{\varepsilon}\right| \leqslant \lambda}\right), \\
& \phi_{\varepsilon}^{>}=\left(1+\varepsilon \partial_{t}+v \cdot \nabla_{x}\right)^{-1}\left(\Phi_{\varepsilon} \mathbb{1}_{\left|\Phi_{\varepsilon}\right|>\lambda}\right) .
\end{aligned}
$$


We then proceed as in [14]. For each $\lambda>0$, the truncated family $\Phi_{\varepsilon} \mathbb{1}_{\left|\Phi_{\varepsilon}\right| \leqslant \lambda}$ is bounded in $L^{2}(M d v d x d t)$ and

$$
\int \frac{|\psi(v)|^{2} M(v) d v}{|1+i \varepsilon \tau+i v \cdot \xi|^{2}} \leqslant \frac{C}{1+\varepsilon|\tau|+|\xi|}
$$

so that the family $\int \phi_{\varepsilon}^{<} \psi M d v$ is bounded in $L_{t}^{2}\left(H_{x}^{1 / 2}\right)$. It therefore satisfies

$$
\left\|\int \phi_{\varepsilon}^{<}(t, x+\delta y, v) \psi(v) M(v) d v-\int \phi_{\varepsilon}^{<}(t, x, v) \psi(v) M(v) d v\right\|_{L_{t, x}^{2}} \leqslant C \sqrt{\delta}
$$

uniformly in $\varepsilon \in[0,1]$.

On the other hand the family $\Phi_{\varepsilon} \mathbb{1}_{\left|\Phi_{\varepsilon}\right|>\lambda}$ satisfies

$$
\left\|\Phi_{\varepsilon} \mathbb{1}_{\left|\Phi_{\varepsilon}\right|>}\right\|_{L_{t, x}^{1}} \rightarrow 0
$$

uniformly in $\varepsilon \in[0,1]$ as $\lambda \rightarrow+\infty$. Therefore

$$
\left\|\int \phi_{\varepsilon}^{>}(t, x+\delta y, v) \psi(v) M(v) d v-\int \phi_{\varepsilon}^{>}(t, x, v) \psi(v) M(v) d v\right\|_{L_{t, x}^{1}} \rightarrow 0
$$

uniformly in $\varepsilon \in[0,1]$ and $\delta>0$ as $\lambda \rightarrow+\infty$. This convergence and the analogous inequality concerning $\phi_{\varepsilon}^{<}$imply that

$$
\left\|\int \phi_{\varepsilon}(t, x+\delta y, v) \psi(v) M(v) d v-\int \phi_{\varepsilon}(t, x, v) \psi(v) M(v) d v\right\|_{L_{t, x}^{1}} \rightarrow 0
$$

uniformly in $\varepsilon \in[0,1]$ as $\delta \rightarrow 0$.

It remains to prove that the same convergence holds in $L_{t, x}^{2}$. We therefore split

$$
\begin{aligned}
\iint\left|\int\left[\phi_{\varepsilon}(t, x+\delta y, v)-\phi_{\varepsilon}(t, x, v)\right] \psi(v) M(v) d v\right|^{2} d x d t \\
\leqslant \iint\left(\int\left|\phi_{\varepsilon} \mathbb{1}_{\left|\phi_{\varepsilon}\right| \leqslant \lambda}\right|(t, x+\delta y, v)|\psi| M d v\right) \\
\quad \times\left|\int\left[\phi_{\varepsilon}(t, x+\delta y, v)-\phi_{\varepsilon}(t, x, v)\right] \psi M d v\right| d x d t \\
+\iint\left(\int\left|\phi_{\varepsilon} \mathbb{1}_{\left|\phi_{\varepsilon}\right| \leqslant \lambda}\right|(t, x, v)|\psi| M d v\right) \\
\quad \times\left|\int\left[\phi_{\varepsilon}(t, x+\delta y, v)-\phi_{\varepsilon}(t, x, v)\right] \psi M d v\right| d x d t \\
+\iint\left(\int\left|\phi_{\varepsilon} \mathbb{1}_{\left|\phi_{\varepsilon}\right|>\lambda}\right|(t, x+\delta y, v)|\psi| M d v\right) \\
\quad \times\left(\int\left[\left|\phi_{\varepsilon}(t, x+\delta y, v)\right|+\left|\phi_{\varepsilon}(t, x, v)\right|\right]|\psi| M d v\right) d x d t \\
+\iint\left(\int\left|\phi_{\varepsilon} \mathbb{1}_{\left|\phi_{\varepsilon}\right|>\lambda}\right|(t, x, v)|\psi| M d v\right)
\end{aligned}
$$




$$
\times\left(\int\left[\left|\phi_{\varepsilon}(t, x+\delta y, v)\right|+\left|\phi_{\varepsilon}(t, x, v)\right|\right]|\psi| M d v\right) d x d t .
$$

The first and second integrals in the right hand side of the inequality above are less than

$$
\lambda\left\|\int \phi_{\varepsilon}(t, x+\delta y, v) \psi(v) M(v) d v-\int \phi_{\varepsilon}(t, x, v) \psi(v) M(v) d v\right\|_{L_{t, x}^{1}},
$$

while the third and the fourth are less than

$$
2\left\|M^{1 / 2} \phi_{\varepsilon} \mathbb{1}_{\left|\phi_{\varepsilon}\right|>\lambda}\right\|_{L_{t, x, v}^{2}}\left\|M^{1 / 2} \phi_{\varepsilon}\right\|_{L_{t, x, v}^{2}} .
$$

This term vanishes as $\lambda \rightarrow+\infty$ uniformly in $\varepsilon \in[0,1]$ since the family $\left|\phi_{\varepsilon}\right|^{2}$ is relatively compact in $\mathrm{w}-L^{1}(M d v d x d t)$. On the other hand, the first and second integrals in (B.2) vanish as $\varepsilon \rightarrow 0$ uniformly in $\lambda>0$ by (B.3) and (B.1). This concludes the proof.

\section{Appendix C. The product limit theorem}

We restate here without proof a corollary of Egorov theorem, established by DiPerna and Lions [12], that was an essential argument in many proofs of this article.

Proposition C.1. - Let $\mu$ be a finite positive measure on the space $\Omega$. Consider two sequences of real-valued measurable functions defined on $\Omega$ denoted $f_{n}$ and $g_{n}$. If $\left(g_{n}\right)$ is bounded in $L^{\infty}(d \mu)$ such that $g_{n} \rightarrow g$ almost everywhere and $f_{n} \rightarrow f$ in $w-L^{1}(d \mu)$ then $f_{n} g_{n} \rightarrow f g$ in $w-L^{1}(d \mu)$, and in $s-L^{1}(d \mu)$ if $g \equiv 0$.

\section{REFERENCES}

[1] Arnold A., Markowich P., Toscani G., Unterreiter A., On logarithmic Sobolev inequalities and the rate of convergence to equilibrium for Fokker-Planck type equations, Comm. PDE 26 (2001) 43-100.

[2] Bhatnagar P., Gross E.P., Krook M., A model for collision processes in gases, Phys. Rev. 94 (1954) 511.

[3] Bardos C., Golse F., Levermore C.D., Fluid dynamic limits of kinetic equations I. Formal derivations, J. Statis. Phys. 63 (1991) 323-344.

[4] Bardos C., Golse F., Levermore C.D., Fluid dynamic limits of kinetic equations II. Convergence proofs for the Boltzmann equation, Comm. Pure Appl. Math. 46 (5) (1993) 667-753.

[5] Bardos C., Golse F., Levermore C.D., Acoustic and Stokes limits for the Boltzmann equation, C. R. Acad. Sci. Paris 327 (3) (1998) 323-328.

[6] Bouchut F., Golse F., Pulvirenti M., in: Kinetic Equations and Asymptotic Theory, in: Perthame B., Desvillettes L. (Eds.), Series in Applied Mathematics, Vol. 4, Gauthier-Villars, Paris, 2000.

[7] CAFlisch R., The fluid dynamic limit of the Boltzmann equation, Comm. Pure Appl. Math. 33 (1980) 651-666.

[8] Cercignani C., The Boltzmann Equation and its Applications, Springer-Verlag, New York, 1988.

[9] Cercignani C., Illner R., Pulvirenti M., The Mathematical Theory of Dilute Gases, in: Applied Mathematical Sciences, Vol. 106, Springer-Verlag, 1994.

[10] Castella F., Perthame B., Estimations de Strichartz pour les équations de transport cinétique, C. R. Acad. Sci. Paris 322 (6) (1996) 535-540.

[11] DeMasi A., Esposito R., Lebowitz J., Incompressible Navier-Stokes and Euler limits of the Boltzmann equation, Comm. Pure Appl. Math. 42 (1990) 1189-1214. 
[12] DiPerna R., LiOns P., On the Cauchy problem for Boltzmann equations: global existence and weak stability, Ann. of Math. 130 (2) (1991) 321-366.

[13] Golse F., LeVermore C.D., Stokes and Acoustic limits for the Boltzmann equation: convergence proofs, Comm. Pure Appl. Math. 55 (3) (2002) 336-393.

[14] Golse F., Lions P., Perthame B., Sentis R., Regularity of the moments of the solution of a transport equation, J. Funct. Anal. 76 (1988) 110-125.

[15] Golse F., Saint-Raymond L., The Navier-Stokes limit of the Boltzmann equation for bounded collision kernels, Inventiones Math., to appear.

[16] Grenier E., Pseudo-differential energy estimates of singular perturbations, Comm. Pure Appl. Math. 50 (9) (1997) 821-865.

[17] Landau L., Lifshitz E., Course of Theoretical Physics, Vol. 6: Fluid Mechanics, Pergamon Press, Oxford, 1987.

[18] LerAy J., Sur le mouvement d'un fluide visqueux emplissant l'espace, Acta Math. 63 (1934) 193-248.

[19] Lions P., Masmoudi N., Une approche locale de la limite incompressible, C. R. Acad. Sci. Paris 329 (1999) 387-392.

[20] Lions P., Masmoudi N., From Boltzmann equations to Navier-Stokes equations I, Archive Rat. Mech. Anal. 158 (2001) 173-193.

[21] Nishida T., Fluid dynamic limit of the nonlinear Boltzmann equation at the level of the compressible Euler equation, Comm. Math. Phys. 61 (1978) 119-168.

[22] Perthame B., Global existence to the BGK model of Boltzmann equation, J. Differential Equations 82 (1989) 191-205.

[23] Philippi P., BRUn R., Kinetic modeling of polyatomic gas mixture, Phys. A. 105 (1981) 147.

[24] Quastel J., YAU H.-T., Lattice gases, large deviations, and the incompressible Navier-Stokes equations, Ann. of Math. (2) 148 (1) (1998) 51-108.

[25] Schochet S., Fast singular limits of Hyperbolic PDEs, J. Differential Equations 114 (1994) 476512.

[26] SaInT-Raymond L., Discrete time Navier-Stokes limit for the BGK Boltzmann equation, Comm. Partial Diff. Equations 27 (2002) 149-184.

[27] SAINT-RAYMOND L., Incompressible hydrodynamic limits for a kinetic model of waves-particles interaction, Asympt. Anal. 19 (2) (1999) 149-183.

[28] Toscani G., Villani C., Sharp entropy dissipation bounds and explicit rate of trend to equilibrium for the spatially homogeneous Boltzmann equation, Comm. Math. Phys. 203 (3) (1999) 667-706.

[29] Welander P., Ark. Phys. 7 (1954) 507.

(Manuscrit reçu le 3 octobre 2001; accepté, après révision, le 4 février 2002.)

\footnotetext{
Laure SAINT-RAYMOND

Laboratoire d'Analyse Numérique,

Université Paris VI,

175 rue du Chevaleret,

75013 Paris, France

E-mail: saintray@ann.jussieu.fr
} 